\title{
CARBON FOAM INFUSED WITH PENTAGLYCERINE FOR THERMAL ENERGY STORAGE APPLICATIONS
}

Thesis

Submitted to

The School of Engineering of the

UNIVERSITY OF DAYTON

In Partial Fulfillment of the Requirements for

The Degree of

Master of Science in Aerospace Engineering

By

Douglas James Johnson

Dayton, Ohio

May, 2011

\begin{tabular}{|} 
UNIVERSITY of \\
DAYTON \\
DATO
\end{tabular} 


\section{CARBON FOAM INFUSED WITH PENTAGLYCERINE FOR THERMAL ENERGY STORAGE APPLICATIONS}

NAME: Johnson, Douglas James

APPROVED BY:

Jamie S. Ervin, Ph.D.

Faculty Advisor
Steven Zabarnick, Ph.D.

Faculty Reader

Paul J. Kreitzer, Ph.D.

Committee Member

John G. Weber, Ph.D.

Associate Dean

School of Engineering
Tony E. Saliba, Ph.D.

Wilke Distinguished Professor \& Dean

School of Engineering 


\begin{abstract}
CARBON FOAM INFUSED WITH PENTAGLYCERINE FOR THERMAL ENERGY STORAGE APPLICATIONS
\end{abstract}

Name: Johnson, Douglas James

University of Dayton

Advisor: Dr. Jamie S. Ervin

A thermal energy storage device that uses pentaglycerine as a phase change material was developed. This solid-state phase change material was embedded in a carbon foam thermal conduction enhancer. This device and others identically constructed but using a paraffin phase change material were tested by imposing different input fluxes, 2.3 or $6.0 \mathrm{~W} / \mathrm{cm}^{2}$ on one end, while the opposite end was either insulated or actively cooled with an output flux that varied from 3.1 to $5.4 \mathrm{~W} / \mathrm{cm}^{2}$. The resulting temperature distributions within the devices were recorded at five locations; this information was used to determine the specific energy storage capacity, heating rate and the cycling performance of each device. It was found that the pentaglycerine/foam combination is capable of a specific storage capacity of $67 \mathrm{~J} / \mathrm{g}$; it demonstrated a storage capacity $174 \%$ of the paraffin/foam device, by eliminating the volume change and leakage problems associated with solid-liquid phase change materials. 
To Mrs. Me 


\section{ACKNOWLEDGEMENTS}

I would like to offer my thanks to my advisor, Dr. Jamie Ervin, for getting me through this project, despite the obstacles; to Dr. Quinn Leland, for suggesting this project in the first place; to Dr. Larry Byrd, for offering simple advice at critical times; to Dave Courson, Stan Hilton, Linda Shafer and Bill Melke for not being afraid to get dirty; to Dr. Paul Kreitzer and Andrew Cole for their critical thinking skills; to Shar'na Clement for laboratory, data reduction and report writing assistance; to Victor Tsao, for LabVIEW wizardry; to Arthur and Lee Wallach, for financial assistance; and especially to the Fisher family: Doug, Jill, Simon, and Claire, for their patience, understanding and generosity; without their kindness, this effort would likely not have happened. 


\section{TABLE OF CONTENTS}

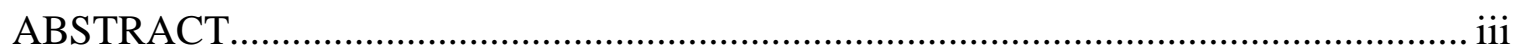

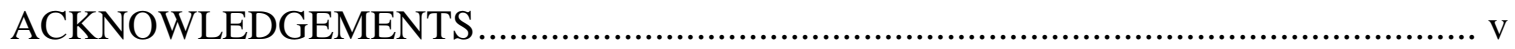

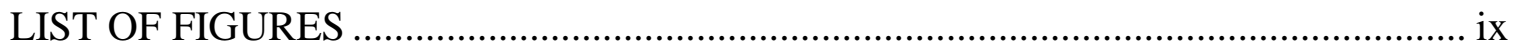

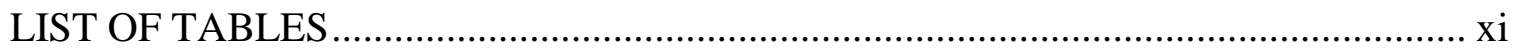

LIST OF ABBREVIATIONS AND SYMBOLS ...................................................... xiii

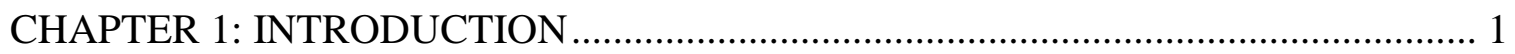

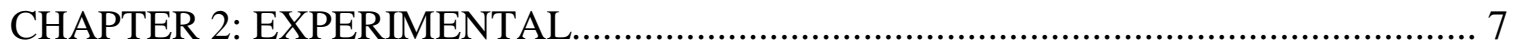

2.1 Sample Construction _ 7

2.2 Infusion _ـ 9

2.3 Sample Holder __ 11

2.4 Experimental Apparatus ___ 13

2.5 Control, Data Recording, and Instrumentation __ 16

2.6 Experimental Procedure __ 19

2.7 Analytical Methods _ 24

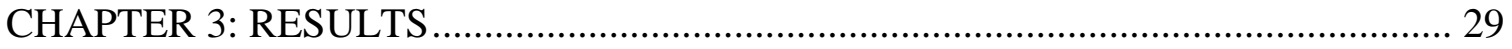

3.1 Material Characterization __ 29

3.1.1 Porosity Measurements ___ 29 
3.1.2 PCM Density, Expansion

3.1.3 PCM Specific Heat Capacities, Transition/Fusion Enthalpies

3.1.4 PG Sublimation/Evaporation

32

3.1.5 Infusion Tests 33

3.2 Experimental Results 35

3.2.1 One-Dimensional Assumption 36

3.2.2 Heating Rates 38

3.2.3 Storage Capacities 42

3.2.4 Cycling Performance 47

CHAPTER 4: CONCLUSION 55

4.1 Summary 55

4.2 Conclusion 55

4.3 Recommendations for Further Study 58

4.3.1 Environmental Stability 58

4.3.2 Temperature Ranges 58

4.3.3 Modeling and Simulation 59

4.3.4 HTC/PG TES Prototypes 59

REFERENCES 60

APPENDIX A: INFUSER MODIFICATIONS 63

A.1 Modification 1: Tetracontane Reinfusion 63

A.2 Modification 2: HTC Porosity Checks 65 APPENDIX B: FOCUS BLOCK THERMAL CONDUCTIVITY. 67

APPENDIX C: UNCERTAINTY ANALYSES. 72

C.1 Thermocouple Calibration and Temperature Measurement Uncertainties 72

C.2 Temperature Difference Uncertainties 74

C.3 Time Step Uncertainty 74

C.4 Temperature Change Rate Uncertainties 76 
C.5 Flux Meter Heat Transfer Uncertainties __ 76

C.6 Sensible Energy Storage Rate Uncertainties __ 77

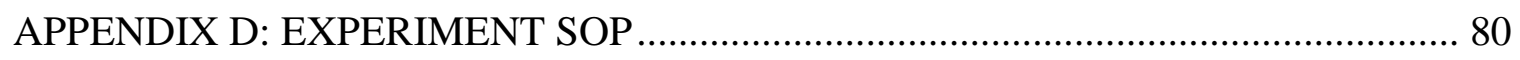

APPENDIX E: SAMPLE CONFIGURATION FILE ........................................... 83 


\section{LIST OF FIGURES}

Figure 2.1: Carbon Foam Sample G0903-H, with Carbon-Carbon End Plates ............................. 8

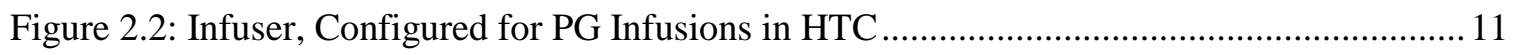

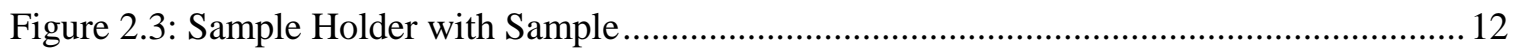

Figure 2.4: Schematic of the Experimental Setup, Showing Major Elements; Active Elements are

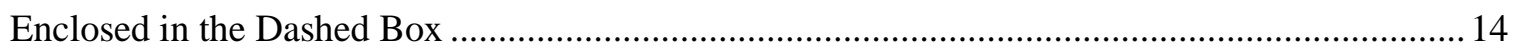

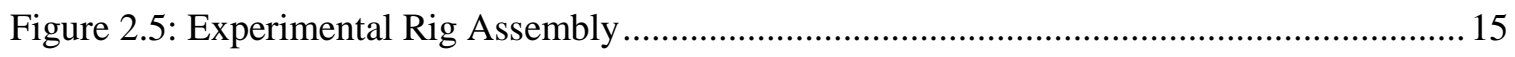

Figure 2.6: Sample Unheated End Boundary Conditions: Insulated (left); Chilled (right) ........... 16

Figure 2.7: Sectional View of Sample G1003-F, Heater Assembly and Insulator Block Showing

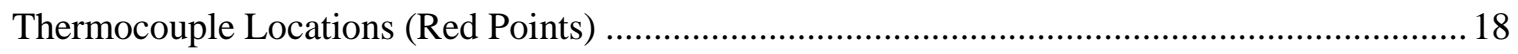

Figure 2.8: Drip Check Setup; $10 \mathrm{ml}$ Beaker on the Balance Contains a $1.0 \mathrm{~cm}^{3}$ "Drip Cube" ...22

Figure 2.9: Sample Hot and Opposite Boundary Temperature Profiles and Interface Heating Rates

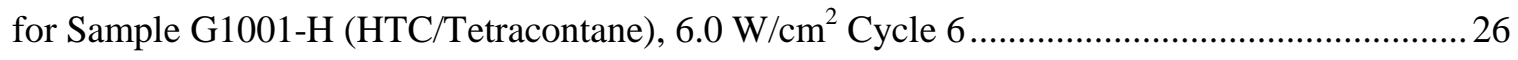

Figure 3. 1: Specific Heat Capacity Curve for Tetracontane; Transition Enthalpy Represented by

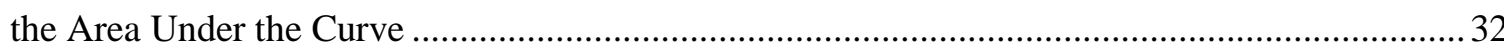

Figure 3.2: DSC Heating and Cooling Curves for PG; Subcooling is Evident in the Displacement

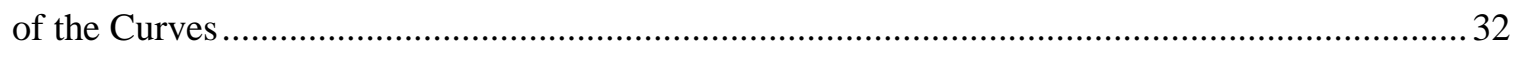

Figure 3.3: Vacuum Oven Sample/PCM Configuration (left); 10X Microphotograph of Sectioned Sample, Showing the Absence of Voids or Empty Pores (center); 15X SEM Photo of PG-Infused HTC Sample, Showing Empty Pores which Appear as Black Regions (right)............................. 34 
Figure 3.4: Sample G1003-F (POCOFoam/HTC) Center Plane Thermocouple Locations for One-

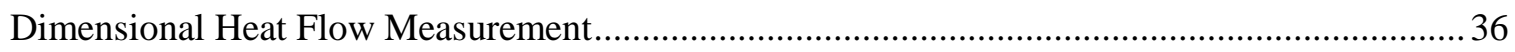

Figure 3.5: Sample G1003-F 6.0 W/cm² Insulated Temperature History through Transition;

Curves Grouped in the Middle are Temperatures on a Common Plane .37

Figure 3.6: Interface Heating Rates Comparison; Each Curve Is From the Last Cycle in Each Run 39

Figure 3.7: Sample and Interface Temperature Profiles for G1002-H, $2.3 \mathrm{~W} / \mathrm{cm}^{2}$ Insulated, Cycle 4. 42

Figure 3.8: Sample G1001-H (HTC/tetracontane) Heating in Different Runs, Identical Conditions; the Storage Capacity in Run 3 Is Lower than in Run 2, Indicating Less PCM Was Available .44

Figure 3.9: Using the Cycle Comparison for the Cold-End Thermocouple of Sample G1002-H to Illustrate the Excess Energy Stored in Sample G1003-F; Cycle 1 Heating is Longer due to System Sensible Storage. .45

Figure 3.10: Power and Sample Temperature Curves for the Complete G1002-H Chilled Run, Showing Four Charge/Discharge Cycles .48

Figure 3.11: Sample G1002-H (HTC/PG) Heated-End Temperature Curves for the Four Cycles, Showing the First Cycle's Longer Transition, Faster Cooling .

Figure 3.12: Sample G1002-H, Cycle 4: Temperature Difference Between Transition Start

Temperature During Heating and Transition Start Temperature during Cooling Is $7^{\circ} \mathrm{C}$ .51

Figure 3.13: Sample Hot-End Temperature Distributions for Samples G1001-H (HTC/wax) and G1002-H (HTC/PG) During the Fourth Cycle of the Chilled Runs; G1002-H Exhibits

Subcooling, G1001-H Does Not .53

Figure 3.14: Samples G0902.1-F (POCOFoam/docosane) and G1003-F (POCOFoam/PG), Chilled Second Cycle; Overall Cycle Times and Duty Cycles Are Similar. .54

Figure A.1: Infuser Piston Showing Burned Packing Seals, after Seven Hours Above $200^{\circ} \mathrm{C} . . . .63$ 
Figure A.2: Sample G1001-H and Copper Sleeve Used for Tetracontane Re-infusion; the Sleeve was Filled with the Remaining PCM and the Sample was Immersed for Infusion .64

Figure A.3: Replacement Plate for Infuser Head; Thermocouple Contacts the Sample..... 65

Figure A.4: Second Infuser Modification, for Infiltrating HTC Drip Cubes; Graduated Cylinder and Water Inlet Valve Are on the Equipment Stand. .66

Figure B.1: Excel Euler Parametric Solver Used to Model the Heater Block Performance. .68

Figure B.2: Solidworks Mesh of Heater Block Assembly; One-Fourth of the Assembly was

Modeled 69

Figure B.3: Solidworks Analysis Results of the $9.0 \mathrm{~W} / \mathrm{cm}^{2}$ Run; Focus Block Conductivity Was

Set to $133 \mathrm{~W} / \mathrm{m}^{\circ} \mathrm{C}$ to Reproduce Experimental Measurements .70

Figure C.1: Residual Plot of the Fourth Order Fits for Thermocouples 1001B-1005B, Used in Sample G1002-H (HTC/PG). 


\section{LIST OF TABLES}

Table 2.1 Carbon Foam Samples Constructed for This Project.................................................. 8

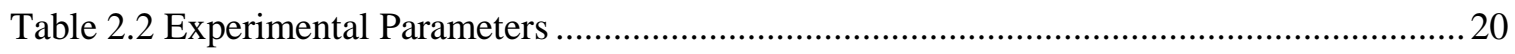

Table 2.3 Specific Heat Capacities and Masses of Sample Components .....................................27

Table 3.1 Heater/Sample Interface Heating Rates for the Insulated Output Experimental Series. 40

Table 3.2 Heater/Sample Interface Heating Rates through Transition, Relative to the HTC/no

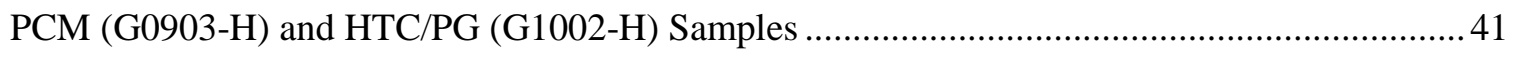

Table 3.3 Total and Specific Latent Storage Capacities for Infused Samples ............................. 43

Table 3.4 Charge / Discharge Times and Rates and Duty Cycles for the Infused Samples ..........52

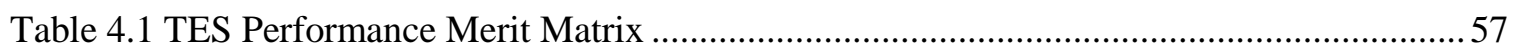




\section{LIST OF ABBREVIATIONS AND SYMBOLS}

\begin{tabular}{|c|c|}
\hline$A$ & area $\left(\mathrm{cm}^{2}\right)$ \\
\hline $\mathrm{BCT}$ & body-centered tetragonal \\
\hline $\mathrm{CI}$ & confidence interval \\
\hline$c$ & specific heat capacity (joules/gram*Kelvin) \\
\hline DAQ & data acquisition unit \\
\hline DSC & differential scanning calorimetry \\
\hline DUL & device under load \\
\hline$E$ & energy (joules) \\
\hline$\dot{E}$ & energy rate, energy per unit time (joules/second) \\
\hline FCC & face-centered cubic \\
\hline HTC & High Thermal Conductivity foam \\
\hline$h$ & enthalpy (joules/gram) \\
\hline$k$ & thermal conductivity (watts/meter*Kelvin) \\
\hline
\end{tabular}




\begin{tabular}{|c|c|}
\hline$m$ & mass (grams) \\
\hline$n$ & population number \\
\hline PCM & phase change material \\
\hline PG & pentaglycerine \\
\hline$q$ & heat power (watts) \\
\hline$q "$ & heat flux (watts/square centimeter) \\
\hline RTD & resistance temperature device \\
\hline RTV & room-temperature vulcanizing silicone \\
\hline SEM & scanning electron microscope \\
\hline SOP & standard operating procedure \\
\hline SSR & sum of squared residuals \\
\hline TES & thermal energy storage \\
\hline TIM & thermal interface material \\
\hline$T$ & temperature (degrees Celsius) \\
\hline$t$ & time (seconds) \\
\hline$V$ & volume (cubic centimeters) \\
\hline VI & virtual instrument (LabVIEW code) \\
\hline$X$ & horizontal lateral direction \\
\hline
\end{tabular}


Y

Z

\section{Greek symbols:}

$\alpha$

$\delta$

$\gamma$

$\varphi$

$\rho$

$\sigma$

\section{Subscripts:}

$\begin{array}{ll}\text { cap } & \text { sample end cap } \\ \text { drift } & \text { random error in thermocouple measurement } \\ f & \text { foam } \\ \text { fit } & \text { accuracy of calibration } \\ i & \text { summation index } \\ \text { in } & \text { input } \\ \text { out } & \text { output } \\ \text { plate } & \text { sample holder end plate }\end{array}$

ambient phase of pentaglycerine

uncertainty

high-temperature phase of pentaglycerine

volume fraction

density (grams/cubic centimeter)

standard deviation 


$\begin{array}{ll}\text { shell } & \text { sample holder shell } \\ \text { st } & \text { stored energy } \\ \text { st, lat } & \text { stored latently } \\ \text { st, sens } & \text { stored sensibly } \\ \text { std } & \text { standard temperature measurement } \\ \text { steady } & \text { standard temperature steady-state range } \\ \text { tr } & \text { transition }\end{array}$




\section{Chapter 1: Introduction}

Thermal energy storage (TES) has been proposed as an alternative to oversized heat rejection systems for aerospace applications. Heat rejection systems tend to be large, heavy and power-intensive. Sizing a heat rejection system for peak loads requires a system that is potentially several times larger than one sized for a continuous, average load. A large waste heat generator that is operated at a low duty cycle, such as a targeting radar, could be coupled with a TES unit. This combination would retain large bursts of thermal energy generated while operating, and then allow a smaller, lighter cooling system to reject the waste heat at a lower average rate. Optimally, the storage unit would be small, light, operate passively, and be easily integrated into existing cooling systems.

The primary performance characteristics of a TES are its specific thermal energy storage capacity with respect to mass or volume, the ability of the TES to maintain the temperature of the device being cooled within the device's operating range, resistance to storage capacity changes due to cycling, and the rate at which the TES can be charged and discharged (Heinz et al. (2004), Zalba et al. (2003), White (2002)). The discharging or energy release performance of a TES device is as important as the charging performance; many phase change materials (PCMs) are known to exhibit subcooling, in that the discharging temperature of the TES can be several degrees below the charging temperature. This may have a direct effect on the duty cycle of the TES. A PCM can also degrade or lose contact with the conduction path due to cycling, affecting the performance of the TES during subsequent charging periods. Characterization of a TES 
device should include thermal cycling studies to determine the existence and effect of these phenomena.

Many TES efforts have focused on storing energy in the latent heat of phase change (Zalba et al. (2003)). By using a material that can be reliably melted and re-solidified, a TES device using this type of PCM can be designed that is compact and passive. To date, a number of PCMs have been investigated or used for aerospace applications (Hale et al. (1971)). The lunar rover of the Apollo program used a paraffin wax TES device to store heat generated by the electronics during driving; the heat was later rejected using radiators after the vehicle had been parked (Morea (1988)). Both salt hydrates and paraffin PCMs have been used successfully despite known issues; for example, salt hydrates do not melt or solidify congruently because the water content of the hydrate changes during each melt/solidification cycle. This can adversely affect the long-term storage capacity and transition temperature of a TES device, according to Farid et al. (2004). Additionally, they indicated that salt hydrates tend to be corrosive, which complicates container material selection. Paraffin waxes exhibit low thermal conductivity, resulting in long cycle times or large temperature gradients for high power applications. Farid et al. (2004) report the large volume expansion paraffins exhibit during melting, on the order of $10 \%$, and they claim the large expansion can lead to high pressures, causing "high stresses on the (container) walls."

To improve the heat transfer through the PCM, designers attempt to maximize the contact surface area between the device being cooled or a heat spreader attached to it and the PCM. This can be done by employing large, thin slabs of PCM which are in thermal contact with the devices or spreaders, or by immersing a material of high thermal conductivity and large surface area into the PCM. The large surface area of the material could take the form of tubes, rods, fins, brushes, foams, or even powders suspended in the PCM. Conduction enhancement using embedded materials does adversely affect the performance of the TES. An attractive characteristic of PCM 
energy storage is that the temperature rise during phase change is small; the energy absorbed is melting the PCM rather than heating it. However, when a conduction enhancer is embedded in the PCM, the temperature of the enhancer will continue to rise even as the PCM changes phase. The inherent temperature regulation of the PCM is offset by this effect. Thus, the design of a TES device using conduction enhancement must account for heating of the conduction enhancing material.

A great deal of interest has been shown lately in using foams for conduction enhancement because of their large surface area per unit volume. Carbon foams, which provide not only the high surface area (on the order of $5000 \mathrm{~m}^{2}$ surface area per $\mathrm{m}^{3}$ bulk volume (Yu et al., 2006)), but also high thermal conductivity (on the order of $180 \mathrm{~W} / \mathrm{m}{ }^{\circ} \mathrm{C}$ (Gallego and Klett, 2003)), have attracted much attention. Harris et al. (2006) characterized the thermal storage performance of infused carbon and aluminum foams using a paraffin; their results indicated that the temperature gradient of the carbon foam/PCM system was about $30 \%$ lower than that of the aluminum foam (typical thermal conductivity value $33 \mathrm{~W} / \mathrm{m}{ }^{\circ} \mathrm{C}$ ) at the same heat flux. Py et al. (2001) conducted experiments using impregnated foams formed by compressing mixtures of wax and graphite powder until the powder formed a conductive matrix. They determined that the bulk conductivity of the resulting matrix was not affected by the presence of the PCM. Mesalhy et al. (2006) performed numerical studies involving carbon foam/PCM assemblies of various porosities and conductivities. They concluded the heat absorption of the various combinations was on average five times higher than a system without foam.

The problem with traditional solid-liquid PCMs like waxes is that they expand considerably during melting, on the order of $10 \%$, and the final product is a liquid. This liquid will tend to migrate out of a foam both by leakage (insufficient capillary action to retain the wax in the pores) and by the expansion itself (the melted wax takes up more volume than the solid wax, so some of it is squeezed out of the pores and has no mechanism to squeeze it back in). This 
PCM migration from the foam degrades TES capacity over time; our studies have shown that as much as $20 \%$ of the initial TES capacity can be lost in only two thermal cycles. A simple solution to this problem is to provide excess volume for the PCM to expand into, and then at least partially fill this excess volume with excess PCM so the foam is always submerged. The container would have to be constructed to handle the pressure generated by the expanding (or contracting) excess PCM if the container is completely filled, but this is not an insurmountable obstacle. An even simpler solution would be to use a PCM that does not melt.

Some researchers have investigated PCMs that either are encapsulated to prevent loss or do not undergo a true solid-liquid phase change. Yang et al. (2003) investigated a microencapsulated paraffin, noting that the material can be embedded in building materials and used as coolant enhancement if suspended in the coolant. They reported that the phase change enthalpy of the encapsulated PCM varied with the hardness of the encapsulant shell. Bach and Haige (2001) investigated a paraffin/plastic mixture in which the paraffin underwent phase change but was retained in the mixture. A heat exchanger filled with the mixture was cycled 70 times and demonstrated a repeatable $86 \mathrm{~kJ}$ storage capacity at $50^{\circ} \mathrm{C}$, indicating no PCM loss. However, the material did shift in the heat exchanger and impede coolant flow. Xiao et al. (2001) describe a $75 / 25$ paraffin/polyethylene mixture that retains $80 \%$ the storage capacity of paraffin alone, but does not melt. They suggest that this material could directly contact coolant flows.

PCMs that do not melt, but instead undergo a solid-solid phase change, have been extensively investigated. Benson et al. (1985) characterized the thermophysical properties of three polyalcohol solid-solid PCMs pentaerythritol (PE), pentaglycerine (PG), and neopentylglycol (NPG), which were known to exhibit high solid-solid transition enthalpies. Chandra et al. (1991) report research on these materials dating back fifty years. 
PG and the other polyalcohols have tetrahedral molecules; for example, the PG molecule has three methylol groups and a single methyl group bonded to the central carbon atom. Because of their terminal hydrogen atoms, these groups form hydrogen bonds across molecules giving the material a crystalline structure. At room temperature ( $\alpha$ phase) PG assumes a body-centered tetragonal (BCT) structure. Planes of PG molecules are held together by the hydrogen bonds, whereas the planes interact through a van der Waals force (Barrio et al. (1994)). As the material is heated through the phase transition temperature, the hydrogen bonds begin to break, so that the individual molecules are then free to rotate and vibrate about their centers. Because of the additional rotational and vibrational states available, the material is able to store thermal energy without heating. Eventually the molecules are attracted only by the van der Waals force ( $\gamma$ phase), and the lattice is considered a face-centered cubic (FCC) configuration. The molecules do not move from their relative positions in the lattice, they simply rotate or vibrate about their centers; this type of structure change is called an orientationally disordered crystalline state (Rudman (1979)). Because the van der Waals forces are so much weaker than the hydrogen bonds, the molecules can be forced under pressure to move against one another, which is why PG and other polyalcohols are referred to as plastic crystals. Barrio et al. (1988) investigated the phase change mechanism of these PCMs, indicating that the reformation of the hydrogen bonds during cooling takes place at a lower energy than the breaking of the bonds during the heating transition. This mechanism causes the subcooling exhibited by these PCMs.

TES research has been conducted using solid-solid PCMs. Zheng and Wirtz (2004) conducted a numerical and experimental study on a heat sink with an embedded PCM blend of PG and NPG. They considered two performance parameters: stabilization time and the ratio between the maximum and transition temperatures. With these parameters, they were able to develop a design optimization algorithm that they then used to design and construct a prototype TES device. Tests conducted on this device focused on storage capacity without considering 
thermal cycling or even discharging. Bauer and Wirtz (2000) constructed a small TES device that used powdered PG as the PCM, packed into a high-porosity aluminum foam. The focus of their study was to measure the effect of the PCM on the thermal conductivity of the foam. They found that the conductivity of the impregnated foam was slightly enhanced by the PCM. They measured the volumetric storage capacity of the device $\left(28 \mathrm{~J} / \mathrm{cm}^{3}\right)$ and subjected the device to a thermal load to confirm energy storage, but did not cycle the TES in order to assess PG subcooling. The low volumetric storage capacity of the TES was due to the low PCM density, a result of the choice to pack powder into the foam rather than fill the foam pores with solid PG.

In this study, we consider a carbon foam (POCO HTC) infused with PG. We believe that this is a novel configuration as no previous studies have been found that consider this combination. A TES device was constructed using these materials, and its thermal energy storage capacity and surface temperature stabilization capability were explored under conditions intended to simulate application. The device was cycled through heat absorption and rejection under various conditions to study potential performance changes due to thermal cycling. The performance of this TES device is compared to the performance of a similarly-constructed device infused with the paraffin tetracontane, which melts at approximately the same temperature as the solid-solid transition of PG. 


\section{Chapter 2: Experimental}

\subsection{Sample Construction}

For this work, several TES samples that rely on carbon foam for conduction enhancement were assembled. The foam of each sample was infused with a different PCM. Each sample was subjected to a constant flux on one surface with either an insulated or cooled boundary on the opposite end. The samples underwent several charge/discharge cycles while the temperature distribution along the sample's centerline, as well as temperatures in other locations, were recorded. The recorded temperature distributions were used to determine the amount of latent energy stored, the sample heating rate, the relative effect of conduction enhancement on the heating rate, and the cycling performance (duty cycle, or charging time/discharging time ratio, and storage capacity degradation due to cycling) of the samples. Conditions were varied for different tests (different input heat fluxes, cooled or insulated opposite boundary) to assist in characterizing the thermal performance of the particular foam/PCM combinations.

Ten samples were prepared, and each sample (Figure 2.1) consists of a foam core with carbon-carbon composite end plates which provide structure and act as heat spreaders. The foams used were POCOFoam and POCO HTC (High Thermal Conductivity), supplied by POCO Graphite. According to the manufacturer, the POCOFoam has a typical bulk thermal conductivity of $90 \mathrm{~W} / \mathrm{m}{ }^{\circ} \mathrm{C}$ and a bulk density of $0.49 \mathrm{~g} / \mathrm{cm}^{3}$, whereas the POCO HTC has a bulk thermal conductivity and density of $188 \mathrm{~W} / \mathrm{m}^{\circ} \mathrm{C}$ and $0.91 \mathrm{~g} / \mathrm{cm}^{3}$, respectively. The cross sections of the samples are $35.8 \mathrm{~mm}$ by $35.8 \mathrm{~mm}$ square. The POCOFoam samples are $41 \mathrm{~mm}$ long including the 
end plates, and the HTC samples are $29 \mathrm{~mm}$ long including the end plates. Table 2.1 lists the materials and weights of the carbon foam samples constructed for this project.

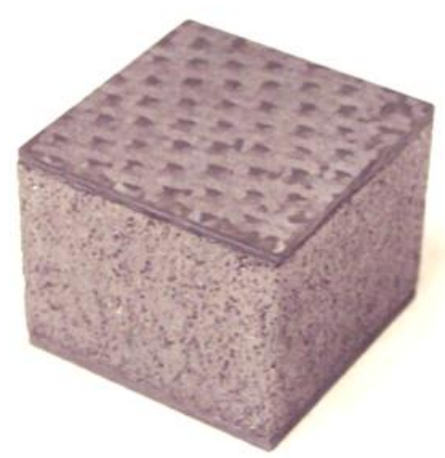

Figure 2.1: Carbon Foam Sample G0903-H, with Carbon-Carbon End Plates

Table 2.1 Carbon Foam Samples Constructed for This Project

\begin{tabular}{ccccc}
\hline $\begin{array}{c}\text { Sample } \\
\text { Name }\end{array}$ & Foam & PCM & $\begin{array}{c}\text { Final } \\
\text { Weight (g) }\end{array}$ & $\begin{array}{c}\text { PCM } \\
\text { Weight (g) }\end{array}$ \\
\hline Glue Test & POCOFoam & None & 34.32 & --- \\
G0901-F & POCOFoam & None & 32.71 & --- \\
G0902.0-F & POCOFoam & Pentaglycerine & 59.58 & 35.26 \\
G0902.1-F & POCOFoam & Docosane & 59.57 & 27.17 \\
G0903-H & POCO HTC & None & 39.92 & --- \\
G1001-H & POCO HTC & Tetracontane & 54.19 & 15.48 \\
G1002-H & POCO HTC & Pentaglycerine & 58.24 & 19.73 \\
G1003-F & POCOFoam & Pentaglycerine & 61.42 & 28.01 \\
G1004-H & POCO HTC & Pentaglycerine & 49.91 & 12.32 \\
G1005-H & POCO HTC & Pentaglycerine & 52.81 & 15.22 \\
\hline
\end{tabular}

The foams were supplied by POCO in the form of 150 x $100 \mathrm{~mm}$ blocks. The POCOFoam block was $38 \mathrm{~mm}$ thick, and the HTC block $25 \mathrm{~mm}$ thick. The sample foam pieces were rough cut from these blocks using a jeweler's saw, and trimmed to their final size using a squaring fixture on a disk sander. The dust generated by sanding was removed by washing the pieces in acetone and blowing dry. A $12.7 \mathrm{~mm}$ spacer was placed in the fixture to raise the HTC pieces beyond the top edge of the fixture for facing. Dimensional accuracy using this fixture was $\pm 0.10 \mathrm{~mm}$. The end plates ( $2 \mathrm{~mm}$ thick AllComp P30) were also sanded to size using the squaring fixture. The end plate material as supplied had a surface roughness caused by the fiber weave 
pattern; the plates were smoothed by sanding, washed with acetone, and wiped dry. All pieces were dried in a vacuum oven at $130^{\circ} \mathrm{C}$. The dried parts were then weighed.

The end plates were attached to the foam pieces with Aremco 551-RN graphite/phenolic high-temperature adhesive. The manufacturer's recommended bond line thickness is between 0.05 and $0.13 \mathrm{~mm}$. It was found that a more consistent adhesive layer could be applied to the plates than to the foam. The pieces were stacked in the squaring fixture used to shape the foam and air dried over night with a $5 \mathrm{~kg}$ weight applied to improve contact. After air-drying, the adhesive was cured at $130^{\circ} \mathrm{C}$ for four hours followed by two hours at $260^{\circ} \mathrm{C}$. Because it was not practical to weigh the adhesive used, that weight was calculated by subtracting the weights of the sample parts from the weight of the completed sample.

\subsection{Infusion}

For TES systems using a metal or carbon foam, the preferred method of infiltrating the foam pores with a PCM is to melt the PCM and immerse the foam. The pores are then filled by capillary action. Some have used vacuum infiltration, in which the foam is evacuated before or during infusion, and then the vacuum is released to allow atmospheric pressure to drive the PCM into the foam (Wierschke et al. (2004)). A modified version of this method was used in this work for the POCOFoam infusions. The vacuum was pulled prior to melting but not released during solidification. Samples were immersed in melted PCM for two to four hours to ensure that all trapped air had time to escape. Infusions of $84 \%$ fill of the POCOFoam were typical. For the high-density, low-porosity carbon foam, POCO HTC, neither evacuation nor atmospheric pressure were sufficient to achieve an acceptable PCM fill. For example, infusing the HTC with docosane using the vacuum oven resulted in the PCM filling only $25 \%$ of the available pore volume. 
The foam manufacturer indicated that success had been achieved by pulling a vacuum on a foam piece and then infiltrating water under high pressure (90 psi). With this method, $100 \%$ of the available volume was filled. Based on this information, a pressure filling method was devised. During a PG infusion, a vacuum could not be used to remove air from the foam due to the high vapor pressure of the melted PG. However, the liquid PG could be forced to flow through the foam with sufficient pressure to displace trapped air. The device developed for this process is shown in Figure 2.2. It consists of a head assembly (1) which has a small restricting hole through which all excess PCM must flow. The head assembly holds the sample (2) and seals it into a melting chamber (3) that has four externally-mounted $20 \mathrm{~W}$ heater elements (4). A pneumatic cylinder (5) drives a Teflon piston in the chamber to force PCM through the sample and the head assembly. The heaters are powered by a digital power supply (HP 6030A) controlled by LabVIEW. The LabVIEW software also controls a data acquisition/control module (Omega USB4718 (6)) which collects thermocouple and pressure transducer data and provides control power for switches and valves (7). A sight glass (8) is mounted on the head assembly to provide a visual indication of PCM flowing through the sample. A typical PG infusion sequence consisted of the following steps outlined below. The chamber was first filled with approximately $100 \mathrm{~g}$ powdered PG; excess material was provided to ensure that all air would be expelled from the sample. The sample was installed in the head assembly, and the sample/head assembly was then sealed in the chamber. The sample was preconditioned by drawing a partial vacuum and heating to $50^{\circ} \mathrm{C}$ for two hours to remove moisture. After preconditioning, the melting sequence starts. A thermocouple embedded in the piston face sensed when the PCM reached the melting temperature $\left(200^{\circ} \mathrm{C}\right)$, and the melted PG was forced through the foam at 90 psi. The infuser was tested twice with PG and POCO HTC before the final HTC/PG and the HTC/tetracontane samples were infused. Modifications to the infuser to complete the HTC/tetracontane infusion are discussed in Appendix A. 


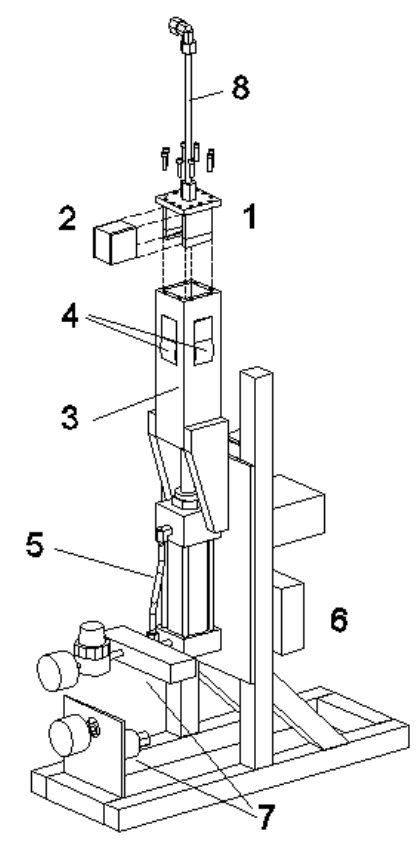

Figure 2.2: Infuser, Configured for PG Infusions in HTC

\subsection{Sample Holder}

This project was originally intended as a continuation of a "wax-in-foam" characterization effort conducted by a different research team (Harris et al. (2006)). One of the most difficult aspects of their project had been developing a method of sealing the samples so that the melted wax would not leak out. Paraffin waxes exhibit significant expansion during melting, on the order of $10 \%$ (Farid et al. (2004)), and will develop considerable pressure if not given sufficient expansion space. The previous effort had focused on encapsulating samples in epoxy. Unfortunately, the wax (docosane) melted and leaked out as it expanded both during epoxy curing and experimental testing. Energy storage capacity results from a sample that lost wax during an experiment were suspect, and that sample could not be used for subsequent tests unless the mass of the remaining wax could be determined. With this in mind, a sample container was developed that would allow disassembly, sample removal and re-infusion. This container would also provide extra volume for wax expansion. The sample would be completely contained; the original sealing method relied on a bond between the epoxy encapsulant and the edges of the 
sample end plates to allow sample sealing yet keep the end plate faces exposed for an uninterrupted thermal path. Wax was routinely lost from these joints.

The new container (the holder) shown in Figure 2.3 is constructed from a $50 \mathrm{~mm}$ square fiberglass tube with $6.35 \mathrm{~mm}$ wall thickness (2) cut to the length of the sample (1), and two end caps (3) made of $2 \mathrm{~mm}$ thick high-purity copper plate. Each end cap is fastened to the fiberglass tube (the shell) using twelve 0-80 flat head screws threaded into tapped holes on the shell ends. O-rings, fitted into slots milled into each end of the shell, were initially used to seal the shell/end cap joints. Each sample required a custom shell, since the sample lengths were not identical and the sample/end cap contact was critical for acceptable heat transfer. The holder shell has a large (12 or $16 \mathrm{~mm}$ diameter, depending on the sample) hole bored in the center of one side for thermocouples to pass through. The sample is positioned in the center of the holder with four polycarbonate shims (4), providing a $1.3 \mathrm{~mm}$ wax expansion gap between the sample and the shell on all four sides. The holder is insulated with $25 \mathrm{~mm}$ thick polystyrene foam (5).

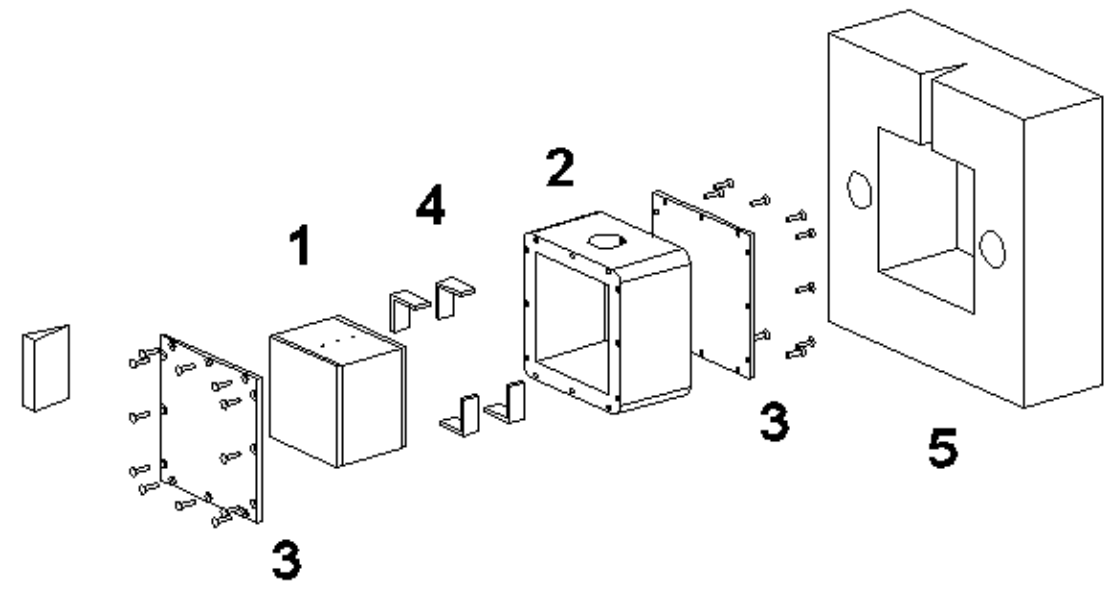

Figure 2.3: Sample Holder with Sample

A problem with the holder design is that it puts material (the end caps, Items 3 in Fig. 2.3) between the sample and the heat supply and removal devices. This was found to be a significant source of thermal resistance during initial tests. For example, the heater face/sample temperature difference was as high as $28^{\circ}$ at the $6.0 \mathrm{~W} / \mathrm{cm}^{2}$ flux level. During a series of tests 
intended to minimize thermal resistances in the holder, a $0.13 \mathrm{~mm}$ thick indium foil thermal interface material (TIM) was inserted between the end caps and the sample end plates. Indium has high thermal conductivity for a TIM $\left(29 \mathrm{~W} / \mathrm{m}^{\circ} \mathrm{C}\right)$ and is very soft so it will conform to eliminate air gaps between the end plate and end cap. This foil is also an excellent gasket material. So, beginning with the third sample, the holder o-ring seals were abandoned and the foil TIM was used to seal the end caps to the holder. Indium foil was chosen for the TIM to avoid contamination of the PCM; it was felt that thermal grease could have changed the performance of the PCM if it migrated onto the sample. Thermal grease was applied between the foil and the end caps to further reduce thermal resistance. It was found that the TIM was reusable if not removed from the end caps. The TIM/thermal grease combination reduced the heater face/sample temperature difference in the same sample by $17^{\circ} \mathrm{C}$ at the $6.0 \mathrm{~W} / \mathrm{cm}^{2}$ flux.

\subsection{Experimental Apparatus}

The experimental apparatus was designed to provide a consistent, controllable set of conditions under which the TES samples were tested and to provide the instrumentation required to record the thermal response of each sample. Figure 2.4 is a schematic showing the connections among the power supply, the data acquisition unit (DAQ), the PC running LabVIEW, and the circulating bath which removes thermal energy from the sample mounted in the experimental rig. As indicated in the schematic, the power supply, DAQ, and circulating bath are computer controlled. The items inside the dashed box are the "active elements" of the experimental rig. They constitute the conductive path through which thermal energy will be added, stored, and removed from the system. Further information on the experimental apparatus can be found in Harris et al. (2006). 


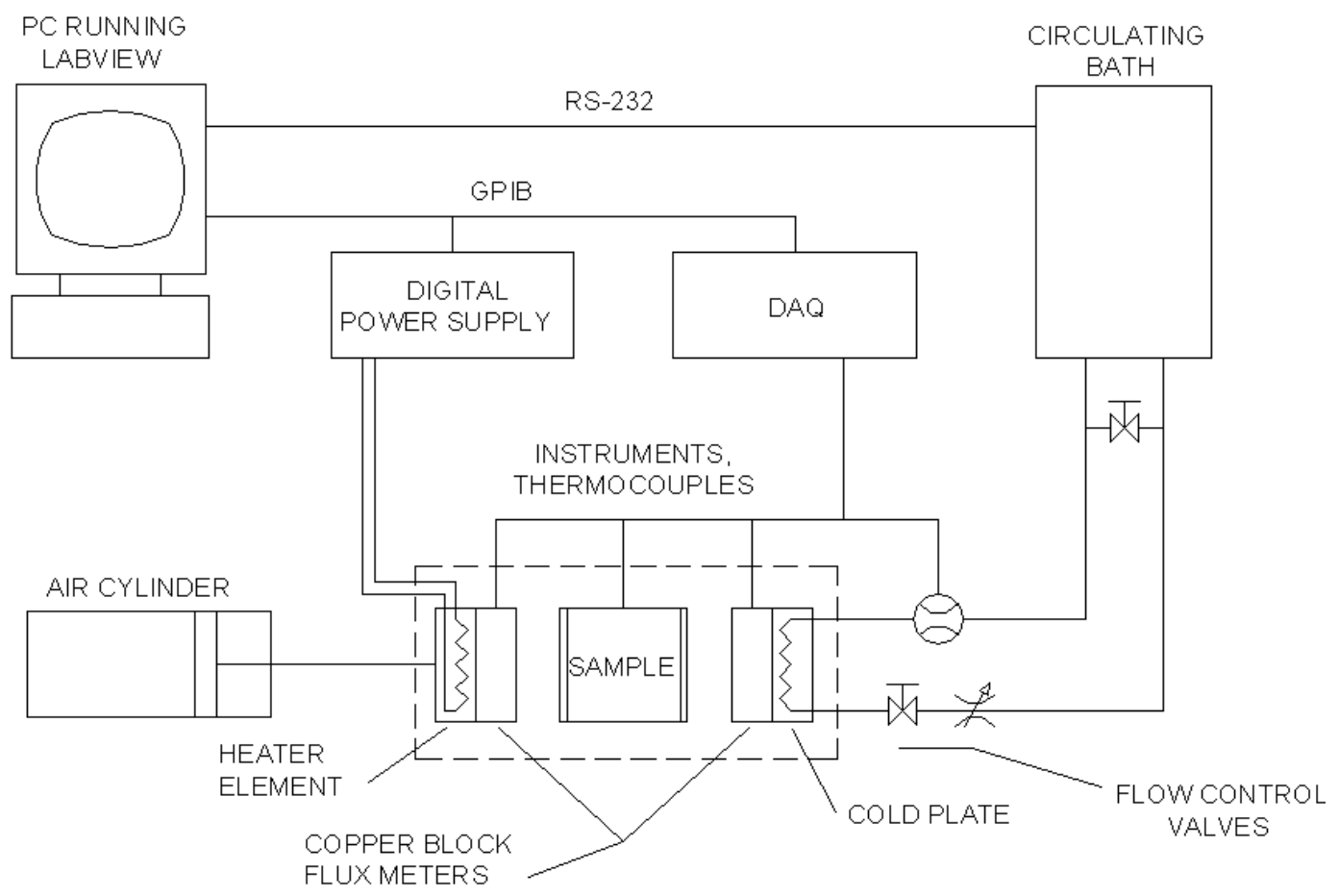

Figure 2.4: Schematic of the Experimental Setup, Showing Major Elements; Active Elements are Enclosed in the Dashed Box

Figure 2.5 shows that the rig consists of a base plate (1), top plate (2), and foot (3) held together with tie bolts (4); guide pins (5) provide proper alignment among active elements: the heater block assembly (6), sample assembly (7) and cold plate with attached flow control valves (8). The top plate-mounted air cylinder (9) generates contact force between the elements. The contact force is controlled by a pressure regulator and measured with a load cell; a repeatable contact force is important for repeatable element contact resistances. The heater block assembly is attached to the air cylinder through a non-conductive ball joint to allow for slight misalignment between the heater block and the sample. The cold plate also contacts the load cell through a ball, again to allow for slight misalignment. During experiments, the active elements were wrapped in a $25 \mathrm{~mm}$ thick ceramic fiber (Kaowool) blanket to minimize heat losses. The experimental rig provided a consistent repeatable heat conduction path through the samples. 


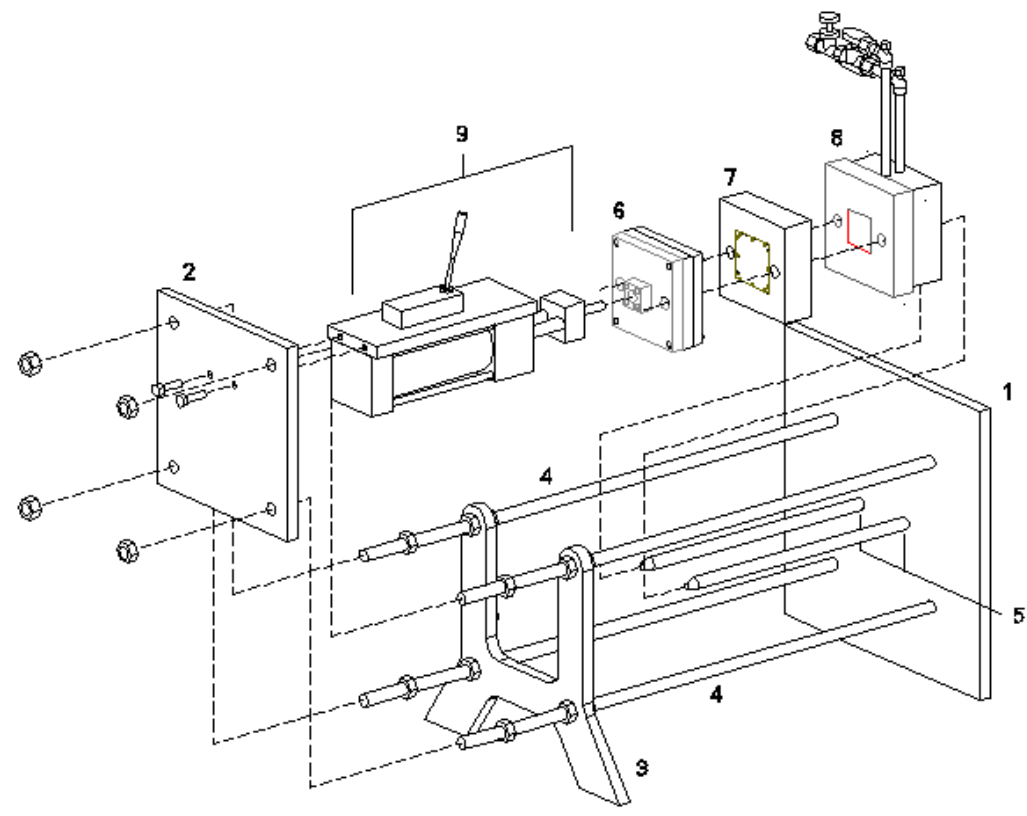

Figure 2.5: Experimental Rig Assembly

Thermal power is supplied to the sample by the heater block assembly (Figure 2.5, Item 6). This assembly consists of a heater element, a focus block, insulation, and a base plate structural member that attaches to the air cylinder. The heater is a kapton film element (Minco HK5590R78.3L12A, $80 \mathrm{~W}$ maximum output) clamped to an oxygen-free copper focus block (12.7 mm thick) with the same face dimensions as the samples. Thermal grease was applied between the heater element and the focus block. The focus block has two embedded $(0.5 \mathrm{~mm}$ type T) thermocouples. The front face thermocouple is $0.25 \mathrm{~mm}$ from the front surface, and the other thermocouple is $0.76 \mathrm{~mm}$ from the back face. The focus block allows a one-dimensional conduction estimate of the heat transferred to the sample. Power to the heater block is supplied by a DC digital power supply (HP 6030A, $1 \mathrm{~kW}$ ) with automatic load sensing.

The experiment can be set up to impose either a cooled or insulated output boundary condition (Figure 2.6). Cooling of the sample was achieved by an offset-fin, one-pass copper cold plate (Figure 2.5, Item 8). The cold plate has a $25 \mathrm{~mm}$ long copper stem attached to the sample side. The stem was used (assuming a 1-D temperature distribution) to determine the heat flux 
with three $(0.25 \mathrm{~mm}$, E-type) thermocouples embedded at $5 \mathrm{~mm}$ intervals. Like the focus block, the cold plate stem face has the same cross-sectional dimensions as the sample faces. Coolant (water) tubes extending from the cold plate have thermocouple probes (1.6 mm, E-type) mounted such that the probe tips are immersed in the coolant. The outlet tube has two valves for adjusting the coolant flow rate. Coolant for the cold plate is supplied by a circulating bath (Thermo Scientific Neslab $17,500 \mathrm{~W}$ ). The flow rate was approximately 0.15 liters per minute at $65^{\circ} \mathrm{C}$. If the insulated boundary condition was required, a $25 \mathrm{~mm}$ thick instrumented block of Marinite-P insulation would be positioned between the sample and the cold plate.
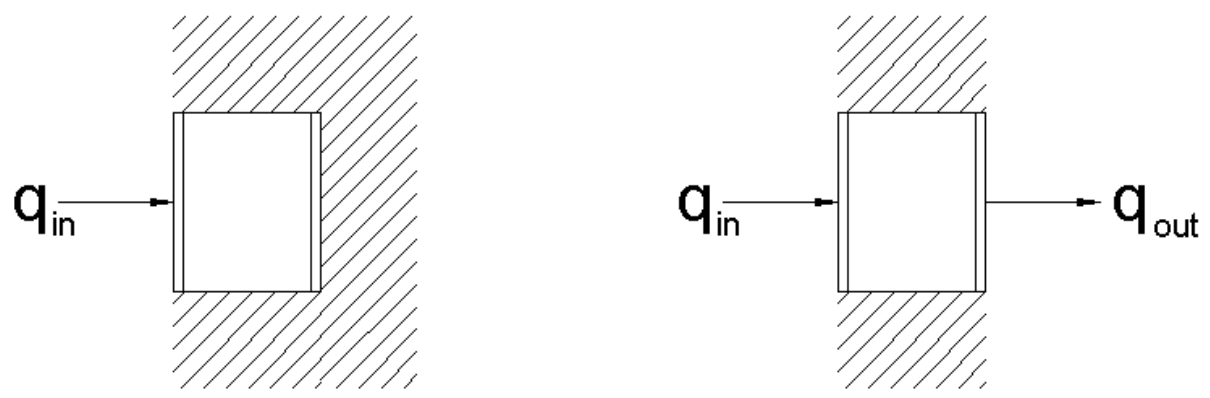

Figure 2.6: Sample Unheated End Boundary Conditions: Insulated (left); Chilled (right)

\subsection{Control, Data Recording, and Instrumentation}

LabVIEW is used for both data acquisition and system control. The LabVIEW code configures the DAQ (Agilent 34980A multifunction switch/measure unit) to record up to 26 channels of temperature, voltage, and frequency data. The code also configures a single selectable channel in the 34980A to act as a monitor channel. For data recording, LabVIEW sets up an Excel spreadsheet file and writes data to it. Each Excel file is configured for the specific experiment performed. Experimental information, such as the sample name, date and time, and experiment parameters are written to one worksheet in the file, and the data from the DAQ are recorded to a second worksheet which is configured with a channel list. The channel list can be configured to include specific channels and apply corresponding calibration equations (if desired) using a configuration file written for the sample and experiment type. A representative 
configuration file is shown in Appendix E. Power data, recorded from the HP 6030A power supply, are recorded on a third worksheet in the same Excel file.

The control functions of the LabVIEW code permit the experimenter to preselect the input power, power cycling information, and bath temperature. The power control function provides two control methods: constant power and constant temperature. In constant power mode, the code commands the power supply to some preset level (e.g., $77 \mathrm{~W}$ ). A pre-selected channel (typically the sample cold-end thermocouple channel) is used to monitor the sample temperature. Once the selected channel reaches a preset temperature, referred to as the trip point, the power is set to zero. The power remains off until the monitor channel temperature reaches the second preset temperature, or recycle point, and the power is again commanded to the preset level. The power cycling continues until the experimenter stops the LabVIEW program. In constant temperature mode, LabVIEW generates power supply commands through an embedded PID subVI; the monitor channel is configured to provide feedback. Constant temperature runs are typically conducted for system and sample characterization studies. The LabVIEW code also controls the circulating bath power and temperature. The bath power control is included to permit configuring experiments in which active cooling is not required.

The instrumentation of the experimental setup is arranged by subassembly or type: sample, environment, heater block, cold plate, voltages and frequency. The sample assembly has up to twelve thermocouple channels assigned. A typical sample has between eight and twelve thermocouples installed. Five thermocouples are located along the sample's central axis, three more at various locations on the sample surface, three on the outer surface of the sample holder and one on the sample insulation surface. The sample axis thermocouples are located at equidistant points along the sample length, with the outermost thermocouples located in the bond line between the foam and the end plates. This arrangement allowed the resulting data to represent the temperature distribution in a control volume that completely contains the foam. The 
environment thermocouples measure the ambient and insulation blanket surface temperatures as well as the removable sample cold-end insulator block, to measure losses from the sample. The heater block has nine thermocouples attached. The two embedded in the focus block have been discussed; two more are on the focus block top side, two are on the insulation edges, one is directly behind the heater element and its complement on the opposite side of the aft insulation, and one is located on the base plate. Using this arrangement, the heater block losses can be accurately characterized. Figure 2.7 shows the thermocouple locations (red circles) in the sample, insulator block and heater block for sample G1003-F (POCOFoam/PG). Also shown in the figure are the relative sizes and arrangement of the components in the sample assembly.

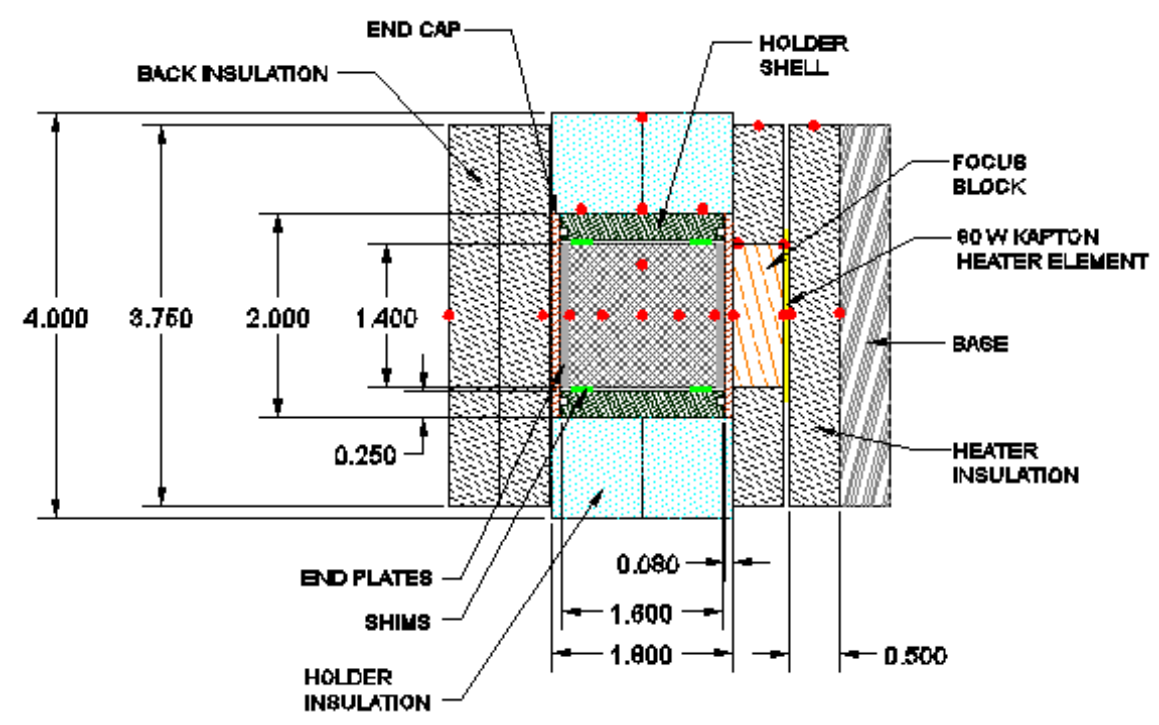

\section{Figure 2.7: Sectional View of Sample G1003-F, Heater Assembly and Insulator Block Showing Thermocouple Locations (Red Points)}

The experimental apparatus has four voltage signals of interest: 10VDC instrumentation power, load cell signal, power supply voltage and power supply current. The power supply voltage and current signals verify the accuracy of the power supply data; the current is measured as the voltage drop across a precision shunt resistor of $0.1 \Omega$. The final signal is generated by the flow meter. The load cell and the flow meter track the consistency of the contact force and the coolant flow rate from one experiment to the next, respectively. 
Installation of the three sample surface thermocouples was discontinued after the third sample test series. Their purpose was to determine whether the heat flow through the sample is one-dimensional; this was accomplished by placing the thermocouples in different locations on a common cross-sectional plane. After the 1-D assumption had been confirmed (Section 3.2.1), the thermocouples were no longer installed.

\subsection{Experimental Procedure}

This project involved the fabrication and testing of a TES device using HTC infused with PG and the fabrication and testing of other TES samples (Table 2.1) required to illustrate the various performance characteristics that are unique to the HTC/PG combination. These characteristics are: 1$)$ the specific storage capacity $(\mathrm{J} / \mathrm{g}) ; 2)$ the interface temperature rise rate; and 3) the sample duty cycle. To study these characteristics, a sample was subjected to various boundary and initial conditions and the temperature distribution within and around the sample before, during and after phase transition were recorded. For the unheated end, the boundary conditions were divided into two types: insulated or chilled. The insulated experiments used the insulator block between the sample and the cold plate. For the chilled conditions the insulator block was removed and the sample contacted with the cold plate which was actively cooled using the circulating bath. The chilled condition resulted in a variable flux output boundary, since no attempt was made to control either the sample output temperature or the output flux. The input condition on the heated surface was always constant heat flux. Two input fluxes were used for experiments in this project, 2.3 or $6.0 \mathrm{~W} / \mathrm{cm}^{2}$. These fluxes were used because they were the input fluxes used for the original effort from which this work was adapted (Harris et al. (2006)), and the data generated here were initially intended to complement the data from the earlier effort. The 6.0 $\mathrm{W} / \mathrm{cm}^{2}$ setting was roughly the highest flux that the heater element could produce, given the

sample cross section. A series of experiments were performed at 3.0 and $9.0 \mathrm{~W} / \mathrm{cm}^{2}$, to 
characterize the heater focus block. These two flux levels were not used to test sample TES performance. The focus block characterization is discussed in Appendix B.

The performance characteristics listed above were determined using the methods described in the Section 2.7 to the temperature distributions generated during the experiments. The TES experiments were conducted according to a standard operating procedure (SOP), which is included as Appendix D. Each sample was subjected to a series of three experiments (two insulated and one chilled), the parameters of which are listed in Table 2.2. The input power was cycled at least four times during each of these experiments to produce cycling performance data.

Table 2.2 Experimental Parameters

\begin{tabular}{cccccc}
\hline Sample & $\begin{array}{c}\text { Input Flux } \\
\left(\mathbf{W} / \mathbf{c m}^{\mathbf{2}}\right)\end{array}$ & $\begin{array}{c}\text { Output } \\
\text { Condition }\end{array}$ & $\begin{array}{c}\text { Bath } \\
\text { Temperature } \\
\left(\mathbf{C}^{\mathbf{C}}\right)\end{array}$ & $\begin{array}{c}\text { Trip } \\
\text { Temperature } \\
\left({ }^{\mathbf{0}} \mathbf{C}\right)\end{array}$ & $\begin{array}{c}\text { Recycle } \\
\text { Temperature } \\
\left({ }^{\mathbf{0}} \mathbf{C}\right)\end{array}$ \\
\hline HTC/wax & 2.3 & Insulated & N/A & 88 & 66 \\
HTC/wax & 6.0 & Insulated & N/A & 88 & 66 \\
HTC/PG & 2.3 & Insulated & N/A & 88 & 66 \\
HTC/PG & 6.0 & Insulated & N/A & 88 & 66 \\
POCOFoam/PG & 2.3 & Insulated & N/A & 88 & 66 \\
POCOFoam/PG & 6.0 & Insulated & N/A & 88 & 66 \\
HTC/no PCM & 2.3 & Insulated & N/A & 88 & 66 \\
HTC/no PCM & 6.0 & Insulated & N/A & 88 & 66 \\
\hline HTC/wax & 6.0 & Chilled & 68 & 88 & 67.5 \\
HTC/PG & 6.0 & Chilled & 68 & 88 & 67.5 \\
POCOFoam/PG & 6.0 & Chilled & 68 & 88 & 67.5 \\
HTC/no PCM & 6.0 & Chilled & 68 & 88 & 67.5 \\
\hline
\end{tabular}

The insulated runs offered a method of easily comparing different heating rates as a PCM undergoes phase change. During the phase change, the sample's temperature rose more slowly than before (pre-transition) or after (post-transition) the phase change. After the PCM had fully transitioned, the sample would resume rapid heating since only sensible storage was available, and because the heat was not being removed by active cooling. The pre-transition and posttransition heating rates could be compared to determine whether there were changes in the 
thermal properties of the different PCM phases, and both were compared to the transition heating rate to determine the effectiveness of the sample's interface temperature regulation performance.

The chilled runs provided two important features. First, they provided consistent cycle starting temperatures and cycle times. The insulated runs could not provide a consistent starting point for subsequent cycles. The system (experimental rig, sample and insulating blanket) heated during cycling; this forced the cycle times to become progressively longer for each run so that the system required more time to cool to a specific temperature. Because one of the goals of the project was to explore cycling performance, the individual cycles had to be as close to identical as possible. Second, the chilled runs provided a measureable output flux through the cold plate stem, making energy storage analyses simpler. Two of the three terms in the energy balance rate equation (Eq. 1 in Section 2.7) can be directly determined from the flux meter temperature differences. The storage rate term is left as the unknown.

One of the arguments for including TES in a thermal management system is that the TES can absorb peak loads, and therefore the heat rejection system (chiller) could be sized for average loads. A goal of this project was to illustrate the peak heating attenuation capacity of the TES. To do this, active cooling of the sample was kept to a minimum during the chilled experiments. The samples were preheated using the circulating bath to what can be considered the average load, and the heater input provided the peak load which was subsequently removed by the bath.

In addition to the TES sample tests, several experiments were conducted to establish the physical properties and fabrication characteristics of the materials used in this project. Some of the properties, such as the density of PG, had published values that spanned a large range. The best way to produce consistent results was to measure the properties and use those values exclusively. 
Considerable effort was given to measure the porosities of the carbon foams. Here, porosity is reported as a percentage of total volume. For example, a foam piece exhibiting a 50\% porosity indicates that half the volume of the piece is available for infusion; the other half is carbon or inaccessible closed pores. (Porosity data were supplied by the foam manufacturer, but what were reported were the open and closed porosities as percentages of total porosity, which gives no indication of the volume available for infusion. It was felt that the available porosity should be measured.) The method developed to measure the porosity (Figure 2.8) was to use a burette to slowly drip a carbon-wetting fluid (methanol) onto a piece of foam with known dimensions and weight. Dripping continued until the piece was saturated, which was indicated by a thin ring of methanol around the bottom of the piece, then the piece was weighed and the fluid volume checked. It was assumed that the available pore volume of the piece would be equal to the volume of fluid used. As a cross check, the piece was filled while on a precision balance (MettlerToledo PB403-S/FACT), so that the weight of the fluid used was also measured. To determine an average porosity, $1.0 \mathrm{~cm}^{3}$ cubes were cut from different depths of the foam. Three cubes were required to span the depth of the POCOFoam. Because of the possible variations in the procedure, such as level of saturation or methanol evaporation, several pieces were each tested many times for statistical validation. Results of the porosity measurements are given in Section 3.1.1.

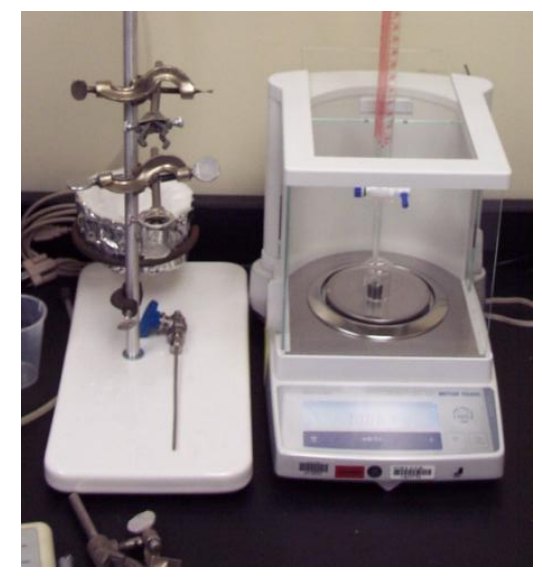

Figure 2.8: Drip Check Setup; $10 \mathrm{ml}$ Beaker on the Balance Contains a $1.0 \mathrm{~cm}^{3}$ "Drip Cube" 
To measure the porosity of HTC the infiltrant fluid (water) was forced into drip cubes under high pressure; the cubes retained the fluid well enough that they could be handled without leaking. The infiltrated cubes were weighed to determine the amount of fluid contained, and the porosity calculated from that value. The infuser modifications and procedure are further considered in Appendix A.

Other physical properties were investigated. One that is reported in literature with some variation is PCM density. Early in this project, it was discovered that sample infusion fill calculations were not yielding accurate results; the calculations were based on published data. An experiment to measure the densities of the PCMs was developed to correct this. The experiment involved filling $2 \mathrm{ml}$ glass syringes with PCMs, and cycling each PCM through melting and solidification. By observing the melting and refreezing of the PCMs and recording their liquid and solid volumes, the thermal expansion coefficients could likewise be determined. The transition enthalpy of PG is another physical property reported with different values: Zheng and Wirtz (2004) report a value of $177 \mathrm{~J} / \mathrm{g}$; Benson et al. (1985) report $192 \mathrm{~J} / \mathrm{g}$, and Sharma et al. (2008) report a value of $216 \mathrm{~J} / \mathrm{g}$. A Mettler-Toledo DSC 823 differential scanning calorimeter (DSC) was used to measure the specific heats and transition enthalpies of the PCMs PG, tetracontane and docosane for this project. Results of these tests are given in Sections 3.1.2 and 3.1.3.

A series of infusion tests were also performed during this project. The difficulties encountered infusing HTC have been discussed; likewise, the vacuum oven method also required several attempts before a successful infusion was achieved. Nine infusion tests were performed during the course of this project; seven using the vacuum oven, and two using the infuser (both discussed in the Section 2.2). Infusion tests were sectioned and the exposed areas examined under a microscope to determine the effectiveness of the respective methods. Infusion test results are given in Section 3.1.5. 


\subsection{Analytical Methods}

Determining the storage capacity of a TES device would seem to be a straightforward exercise; it is the product of the mass of the PCM in the sample and the transition enthalpy of the PCM. However in a TES that employs conduction enhancement, the specific heat capacity of the foam contributes to the overall storage capacity. The foam not only affects the storage capacity, it

affects the temperature regulation behavior of the PCM. As the PCM is storing energy by changing phase, the foam is storing energy by sensible heating. A foam/PCM combination therefore should exhibit a characteristic heating rate during the phase change.

The rate at which the temperature of the device under load (DUL)/TES interface rises is an important aspect of TES design. If the rate is too high, it is possible the DUL will exceed its operating temperature limit before the TES has fully charged. From previous work (Johnson et al. (2009)), it has been shown that using low-thermal conductivity foams for conduction enhancement can have an adverse effect on the interface heating rate. It was felt that the sample interface heating rates should be investigated.

Conduction problems, such as phase-change thermal energy storage, can be analyzed by first considering energy conservation. The energy balance on a rate basis for a system is

$$
\dot{E}_{\text {in }}=\dot{E}_{\text {out }}+\dot{E}_{\text {st }}
$$

In this case, considering the sample to be the system and assuming perfect insulation,

$$
\dot{E}_{\text {in }}=q_{\text {in }}
$$

where $q_{\text {in }}$ is the thermal power supplied to the sample by the heater focus block; and

$$
\dot{E}_{\text {out }}=q_{\text {out }}
$$


where $q_{\text {out }}$ is the power extracted by the cold plate. The energy storage rate term $\dot{E}_{s t}$ is a combination of the sensible and latent storage rate terms (the latent storage term must be divided by the latent storage time $t_{t r}$ if the rate is to be found):

$$
\begin{gathered}
\dot{E}_{s t}=\dot{E}_{s t, s e n s}+\dot{E}_{s t, l a t} \\
\dot{E}_{s t}=\sum_{i} m_{i} c_{i} \frac{\partial T_{i}}{\partial t}+\frac{1}{t_{t r}} m_{P C M} h_{t r}
\end{gathered}
$$

The summation is used to account for the thermophysical properties of the different materials (assumed constant) contained in the sample. The volumetric heating rate can be found by letting $\varphi_{i}$ be the volumetric fraction of the $i^{\text {th }}$ component, and summing:

$$
\frac{\partial T}{\partial t}=\left[\sum_{i} \rho_{i} \varphi_{i} c_{i}\right]^{-1}\left[\left(q_{z}^{\prime \prime}-q_{z+d z}^{\prime \prime}\right)-\frac{1}{t_{t r}} \varphi_{P C M} \rho_{P C M} h_{t r}\right]
$$

Thus by knowing the thermophysical properties of the materials and measuring the transition time and the input and output fluxes, the volumetric heating rate can be found.

For this effort, the heating rates of the samples were found from the experimental temperature distribution data. A heating rate is found by taking the temperature difference of successive time steps of a particular thermocouple $j$ and dividing by the average time step during the period of powered operation:

$$
\frac{d T_{j}}{d t}=\frac{T_{j, t+1}-T_{j, t}}{\overline{\Delta t}}
$$

Plotting the resulting heating rate data allows for visual confirmation of the phase transition start and end points; these are marked by sharp slope changes (Figure 2.9). Using the rate data plots, the rate data for a sample in a particular cycle were divided into three regions: pre-transition, transition, and post-transition. The rate values over these regions were averaged to give a single value for heating rate per region. The temperature rise rate of the heater focus block face 
thermocouple is taken to be the interface heating rate. This thermocouple is common to all the experiments, and therefore is a good gauge of sample heating performance. Multiplying the interface heating rate by the transition time should give the expected DUL temperature rise during transition. The results of the interface heating analyses are given in Section 3.2.2.

Transition times were found by determining the elapsed time from which the sample heated-end thermocouple reached approximately $80.0^{\circ} \mathrm{C}$ for the PG samples G1002-H (HTC/PG) and G1003-F (POCOFoam/PG), or approximately $78.5^{\circ} \mathrm{C}$ for G1001-H (HTC/tetracontane), until the sample cold-end thermocouple reached approximately $84.3^{\circ} \mathrm{C}$ for the $\mathrm{PG}$ samples or approximately $82.5^{\circ} \mathrm{C}$ for G1001-H (Figure 2.9). These temperatures were selected by crossreferencing the heating rate data plots per sample, per cycle; they are average values based on the plots. Figure 2.9 shows two curves from a typical sample temperature distribution (upper curves) with the heating rates overlaid (lower curves). The regions of large positive or negative slope in the lower curves indicate the start and end of phase transition. The highest points of the largeslope regions (points 35 and 105 on the $\mathrm{x}$-axis in Figure 2.9) were chosen to correspond to the selected transition start and end temperatures.

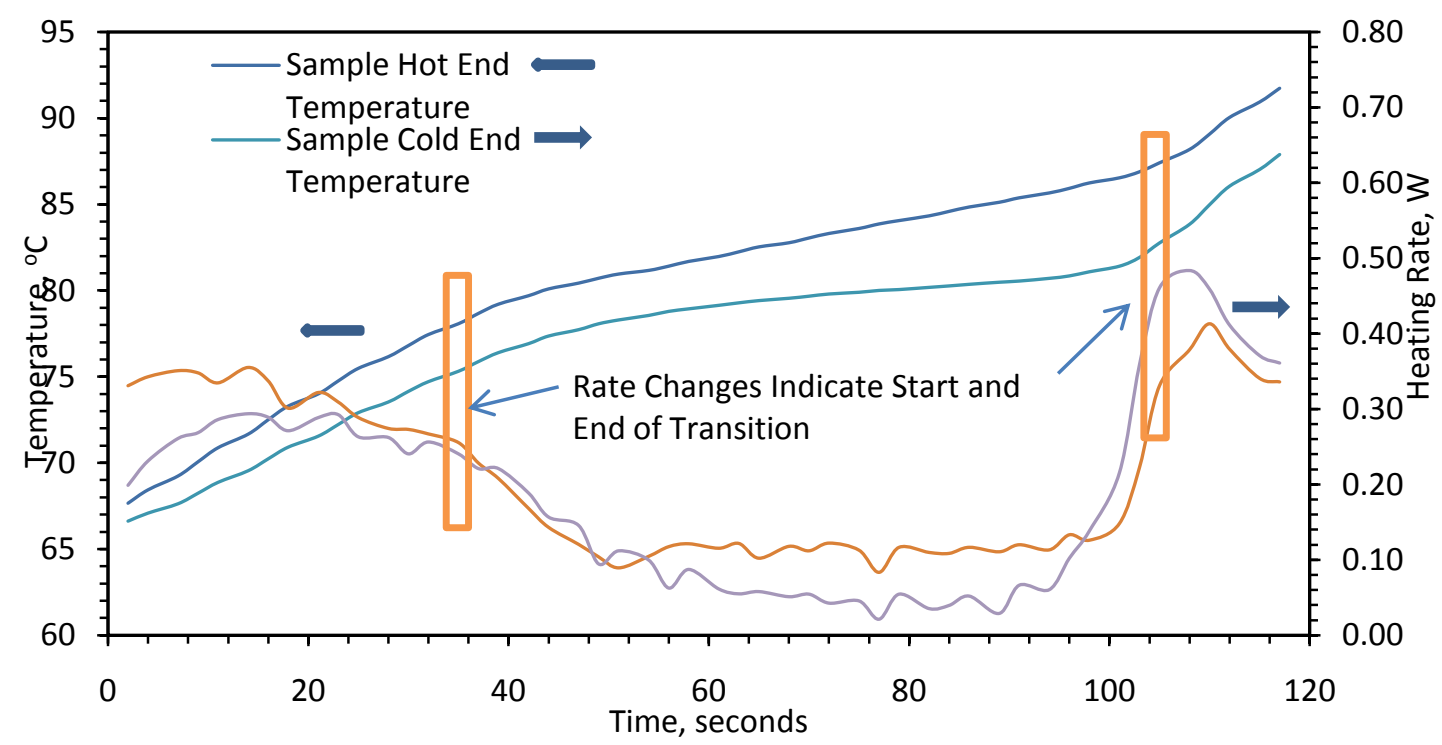

Figure 2.9: Sample Hot and Opposite Boundary Temperature Profiles and Interface Heating Rates for Sample G1001-H (HTC/Tetracontane), 6.0 W/cm² Cycle 6 
The experiments used to find the storage capacities are labeled "Chilled" in Table 2.2; these experiments provided the means to measure both the input and output heat transfer rates. Substituting definitions (Eqs. 2-4) into Equation 1and rearranging,

$$
E_{\text {st,lat }}=t_{t r}\left[q_{\text {in }}-\left(q_{\text {out }}+\sum_{i} m_{i} c_{i} \frac{d T_{i}}{d t}\right)\right]
$$

where the heat transfer rates $q$ are found by

$$
q=\frac{k A}{L}\left(T_{1}-T_{2}\right)
$$

This indicates that by measuring the input and output heat transfer rates and the sensible storage terms in the sum, subtracting appropriately and multiplying by the transition time, the latent storage capacity can be found. The sensible storage terms require the masses and specific heat capacities of the all the components in the thermal path; these are listed in Table 2.3. The method used to find the differential time steps used in calculating the sensible storage terms is the same as for the heating rates, using Equation 6. Results of the storage capacity analyses are given in Section 3.2.3.

Table 2.3 Specific Heat Capacities and Masses of Sample Components

\begin{tabular}{cccccc}
\hline Component & $\begin{array}{c}\text { specific heat } \\
\left(\mathbf{J} / \mathbf{g}^{\mathbf{0}} \mathbf{C}\right)\end{array}$ & $\begin{array}{c}\text { G0903-H } \\
\text { Mass }(\mathbf{g})\end{array}$ & $\begin{array}{c}\text { G1001-H } \\
\text { Mass }(\mathbf{g})\end{array}$ & $\begin{array}{c}\text { G1002-H } \\
\text { Mass }(\mathbf{g})\end{array}$ & $\begin{array}{c}\text { G1003-F } \\
\text { Mass }(\mathbf{g})\end{array}$ \\
\hline End Caps & 0.385 & 93.08 & 92.20 & 92.08 & 92.73 \\
TIM (average) & 0.238 & 4.64 & 4.64 & 4.64 & 4.64 \\
End Plates & 0.7 & 8.79 & 9.04 & 9.14 & 8.95 \\
Foam & 0.7 & 28.23 & 28.79 & 28.77 & 24.66 \\
PG & 2.75 & --- & --- & 19.73 & 28.01 \\
Tetracontane & 2.89 & --- & 15.48 & --- & -- \\
Shell & 1.2 & 57.7 & 57.9 & 57.2 & 81.08 \\
\hline
\end{tabular}

The cycling performance is investigated by measuring the times required to charge and discharge the samples. Due to the differences in heat input and heat removal in typical TES applications, charging time is much shorter than discharging time; that is, a TES device is 
operated at a low duty cycle. Therefore, characterizing the duty cycle of a particular TES configuration was considered important. Cycling performance results are given in Section 3.2.4. Qualitative analyses were also performed; data from individual cycles generated during a particular run were plotted together to assess cycle differences. This qualitative assessment was done for the insulated experimental series as well as the chilled series.

An important task for this project was the determination of the percentage of PCM fill for an infusion. While it is possible to determine the thermal storage capacity of a TES device from the quantity of PCM used, knowing that quantity gives no indication of the extent to which the foam pores are filled. An optimized TES device will have all the available pores filled with PCM. Determining the fill percentage requires the mass and volume of the TES foam, $m_{f}$ and $V_{f}$; the porosity of the foam, $\varphi_{f}$; the density of the PCM, $\rho_{P C M}$; and the mass of the TES sample, $m_{T E S}$. The theoretical maximum mass of PCM that the foam can contain, $m_{P C M, 100}$ is found by

$$
V_{f} \varphi_{f} \rho_{P C M}=m_{P C M, 100}
$$

The mass of the PCM used is

$$
m_{T E S}-m_{f}=m_{P C M}
$$

Therefore, the percentage of fill is the ratio of the PCM mass contained to the theoretical PCM mass,

$$
\frac{m_{P C M}}{m_{P C M, 100}}=\% \text { fill }
$$

For example a $75 \%$ fill indicates that the sample contains $75 \%$ of the PCM it could contain if all available pores were filled. 


\section{Chapter 3: Results}

\subsection{Material Characterization}

Several material properties were measured to provide consistent property values. The analytical methods used to determine calculated values are described in Section 2.7.

\subsubsection{Porosity Measurements}

The porosity of the POCOFoam and POCO HTC were measured using $1.0 \mathrm{~cm}^{3}$ cubes, which were infiltrated with a fluid using the method previously described. Several sets of cubes were cut from several full-depth pieces, so the porosity variation along the depth would be represented by the different cubes in any one set. Nine POCOFoam cubes were tested. It was found that the porosity varied along the depth of the foam block, and therefore the POCOFoam test samples, from $59 \%$ at the bottom to $87 \%$ at the top. The average bulk porosity was found to be $65.9 \%$, which is an $11 \%$ difference from the manufacturer's stated porosity value of $74 \%$. The 65.9\% porosity value was used for sample G1003-F (POCOFoam/PG).

During the porosity measurements, it was discovered that HTC could not be infiltrated by the drip method. Several attempts were made, and the average porosity value found was $32 \%$, with a range from $27 \%$ to $36 \%$, which did not compare well with the manufacturer's porosity value of 59\%. The HTC cubes were later infiltrated with water using the infuser, which had been

modified to re-infuse sample G1001-H (HTC/wax). Three cubes were tested; the average porosity 
found using this method was $54.5 \%$, within $8 \%$ of the manufacturer's porosity value. The $54.5 \%$ porosity value was used for samples G1001-H (HTC/wax) and G1002-H (HTC/PG).

\subsubsection{PCM Density, Expansion}

In the literature, there exists a range of reported density values for PG. Hale et al. (1971) reported $1.16 \mathrm{~g} / \mathrm{cm}^{3}$; Benson et al. (1985) reported $1.22 \mathrm{~g} / \mathrm{cm}^{3}$. Many sources, such as Zheng and Wirtz (2004) report a value between 0.75 and $0.78 \mathrm{~g} / \mathrm{cm}^{3}$. This value represents the density of powdered PG. The density value that would be used for this work had to be determined independently.

The needle end of a $2.0 \mathrm{ml}$ glass syringe was plugged with RTV, the plunger removed, and the weight of the syringe was recorded $(11.16 \mathrm{~g})$. The syringe was filled with $1.7 \mathrm{ml}$ powdered PG, which was then packed using the plunger. The plunger was removed and the syringe weighed again (12.58 g). The PG was melted (with the plunger sealing the end of the barrel to eliminate sublimation/evaporation losses) and re-solidified. After solidification, the surface of the solid PG was shaved until the volume measured a marked quantity, in this case 1.2 $\mathrm{ml}$. The syringe containing the known volume weighed $12.54 \mathrm{~g}$, giving a contained PG weight of $1.38 \mathrm{~g}$; the resulting density was $1.15 \mathrm{~g} / \mathrm{cm}^{3}$. This value was used for all PG-related calculations in this project. This same procedure was used to find the density of tetracontane; $1.0 \mathrm{ml}(0.87 \mathrm{~g})$ was added to the syringe and it was cycled through melting and refreezing. The density was found to be $0.87 \mathrm{~g} / \mathrm{cm}^{3}$ for solid tetracontane.

Using syringes filled with melted PCM also provided a method of measuring the expansion of the PCMs. For PG, this test was performed after the density measurement test. The plunger was replaced; the syringe reheated to above transition temperature, in this case $106^{\circ} \mathrm{C}$. At that temperature, the plunger/PG line expanded above the $1.2 \mathrm{ml}$ mark, to what was estimated to be $1.23 \pm 0.02 \mathrm{ml}$. This gave an expansion of $2.5 \%$. Because of the uncertainty in the 
measurement, the expansion could have been as high as 3.8\%. This result is in marked contrast to some published reports. For example, Hale et al. (1971) report "volume changes are usually of the order of 10 to $50 \%$ " for solid-solid organic PCMs such as PG. This test was also performed on the tetracontane during the density measurement melt/freeze cycle. At $83.8^{\circ} \mathrm{C}$, the volume of melted tetracontane was $1.1 \mathrm{ml}$. The resulting density was $0.79 \mathrm{~g} / \mathrm{cm}^{3}$ which indicated an expansion of $10 \%$. This is within the range reported in the literature (Farid et al. (2004)).

\subsubsection{PCM Specific Heat Capacities, Transition/Fusion Enthalpies}

The specific heat capacities of PG and tetracontane were measured using differential scanning calorimetry (DSC) for this study. Figure 3.1 shows the specific heat capacity $\left(\mathrm{c}_{\mathrm{p}}\right)$ curve generated for tetracontane, generated at a rate of $1.0^{\circ} \mathrm{C} /$ minute using a sapphire standard. The phase transition (peak) begins at about $80.5^{\circ} \mathrm{C}$ and ends at about $82.2^{\circ} \mathrm{C}$, peaking at $81.3^{\circ} \mathrm{C}$. The transition enthalpy is equal to the area under the curve; from the plot, the calculated value for the transition enthalpy of tetracontane is $210 \mathrm{~J} / \mathrm{g}$. The peak temperature and transition enthalpy values compare well with the published values of $81.4^{\circ} \mathrm{C}$ and $237 \mathrm{~J} / \mathrm{g}$ (Lourdin et al. (1992)). Figure 3.2 shows a plot of the DSC data for $\mathrm{PG}$, also recorded at $1.0^{\circ} \mathrm{C} /$ minute; the upper curve represents the change in specific heat during cooling, and the lower curve represents the change during heating. The two curves do not cover the same temperature ranges as PG exhibits significant subcooling. PG begins transition during heating around $80^{\circ} \mathrm{C}$ and ends around $87^{\circ} \mathrm{C}$, peaking at $85.1^{\circ} \mathrm{C}$. During cooling, transition begins around $73^{\circ} \mathrm{C}$ and ends around $64^{\circ} \mathrm{C}$, peaking at $70.3^{\circ} \mathrm{C}$. The transition enthalpy of PG has some variation in the literature. For example, Wilmet et al. (1990) report a value of $145 \mathrm{~J} / \mathrm{g}$, Benson et al. (1985) report $192 \mathrm{~J} / \mathrm{g}$, and Sharma et al. (2008) report a value of $216 \mathrm{~J} / \mathrm{g}$. Based on the DSC data, the transition enthalpy of PG is $157 \mathrm{~J} / \mathrm{g}$, which is in the range reported in the literature; the heating transition temperature is also within the range found in the literature, which is reported from $81^{\circ} \mathrm{C}$ (Hale et al. (1971)) to $89^{\circ} \mathrm{C}$ (Benson et al. (1985)). Cooling transition temperatures do not appear to be reported. The effect of the uneven 
cooling transition enthalpy can be seen in the low-rate discharge temperature histories of sample G1003-F (POCOFoam/PG); the temperature of the sample rises slightly after transition begins, and then gradually falls off.

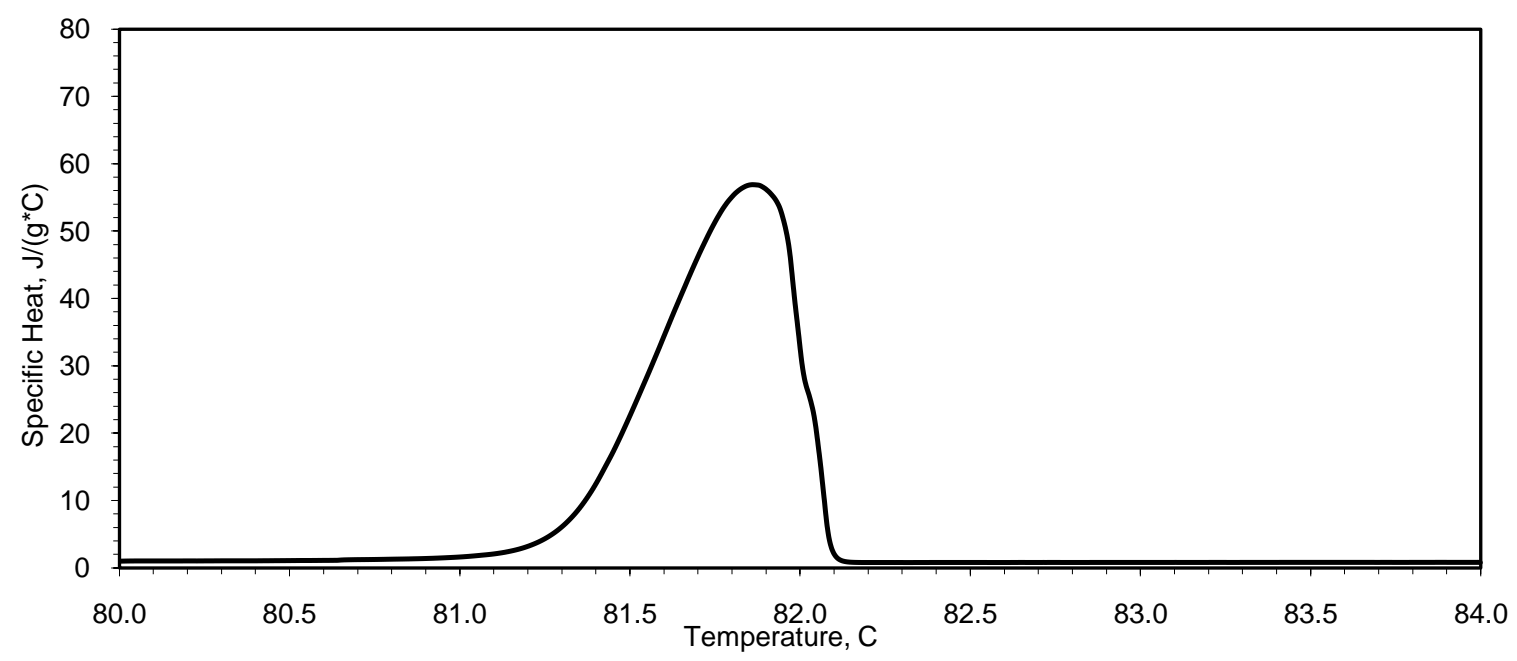

Figure 3. 1: Specific Heat Capacity Curve for Tetracontane; Transition Enthalpy Represented by the Area Under the Curve

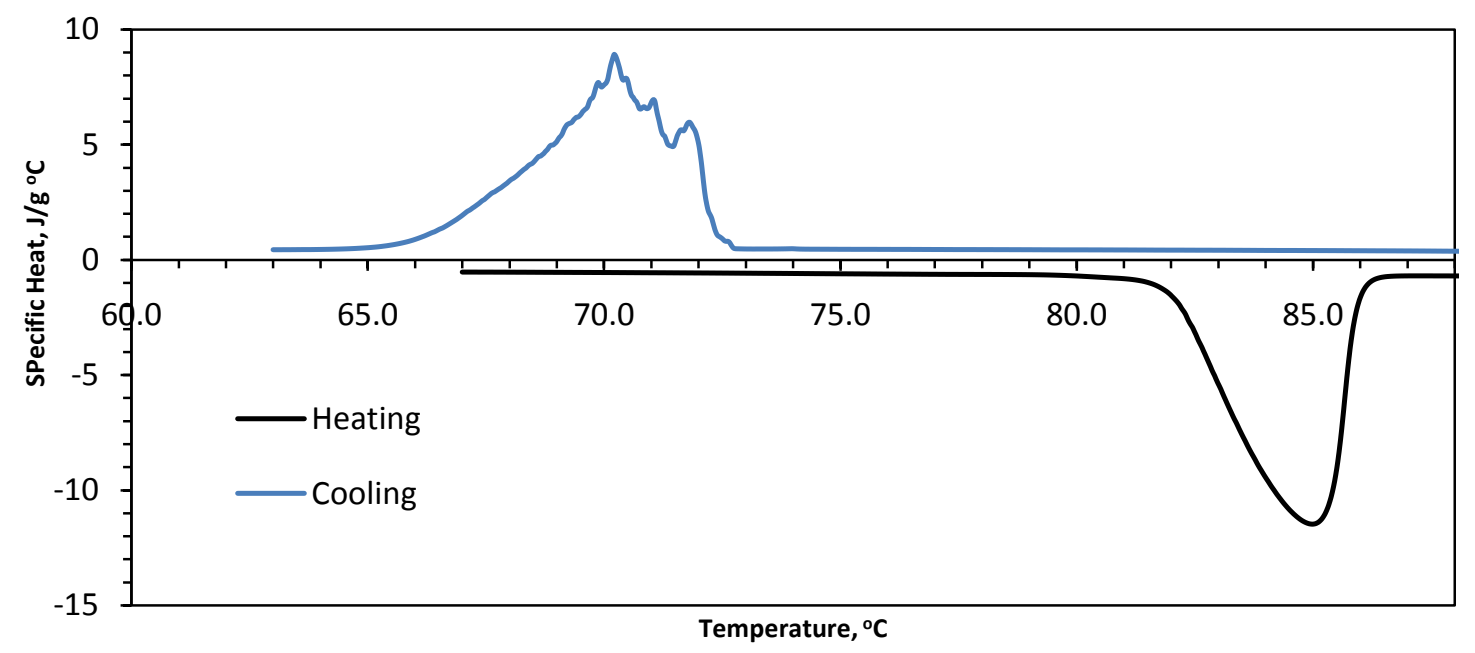

Figure 3.2: DSC Heating and Cooling Curves for PG; Subcooling is Evident in the Displacement of the Curves

\subsubsection{PG Sublimation/Evaporation}

Benson et al. (1985) describe the high vapor pressure of PG "near and above (its) melting temperature." Because the initial infusion plan included drawing a vacuum to remove air from the 
sample during PCM melting, it was felt that a test should be performed to determine the amount of material that would sublimate or evaporate during infusion.

The sublimation/evaporation testing was done during the first infusion test of PG in POCOFoam. Two drip cubes were infused with PG using the vacuum oven: $1.67 \mathrm{~g}$ PG in the first beaker, with an overall weight (beaker, foam, PCM) of $11.14 \mathrm{~g} ; 1.99 \mathrm{~g} \mathrm{PG}$ in the second, with an overall weight of $11.16 \mathrm{~g}$. After the PCM had completely melted (oven temperature $206^{\circ} \mathrm{C}$ ), a partial vacuum (10" Hg on the oven display) was drawn. The samples were allowed to solidify overnight in the partial vacuum. On examination the following morning, PG had deposited on the oven window; the first beaker lost $0.64 \mathrm{~g}$, and the second lost $0.65 \mathrm{~g}$, for an average loss of 35.5\%. A subsequent test indicated that the PCM did not need to be melted (but still heated to about $100^{\circ} \mathrm{C}$, within the expected samples' operating range) for noticeable loss to occur. A third test was done to determine the highest vacuum/temperature combination that could be used to precondition the foam without losing PCM. It was found that a vacuum at 5" $\mathrm{Hg}$ could be drawn and the material heated to $50^{\circ} \mathrm{C}$ for several hours without detectable PCM loss. These settings were later used to precondition samples during PG infusion.

\subsubsection{Infusion Tests}

The goal for the infusion tests was to demonstrate and verify an effective method of infusion. Nine vacuum oven and two infuser tests were performed to determine whether this goal had been met.

For the vacuum oven tests, the primary concern was how to configure the solid PCM and foam in the oven to ensure that the foam would be exposed to vacuum during melting and be fully submerged after melting. The configuration used (Figure 3.3 (left)) was a sample placed end plate down and 80-100 $\mathrm{g}$ of PCM in a $200 \mathrm{ml}$ beaker, with the PCM formed into two 40-50 g pieces that fit loosely into the beaker, located on the sample. As the PCM melted, it filled the beaker, 
submerging the sample. The samples would typically remain in melted PCM for two to four hours, to allow trapped air to escape. To verify whether the vacuum oven infusion method was effective, one infusion test sample was milled in layers, and each exposed surface examined under a microscope. Eight layers, each $2.5 \mathrm{~mm}$ deep, were removed from the sample; no significant voids were observed, even at the center plane of the sample. Figure 3.3 (center) shows a 10X microscope photo of the cut at a depth of $15 \mathrm{~mm}$; no voids are visible, and it appears that all the pores are completely filled. In addition to the microphotographs taken of the milled sample, foam pieces infused with PG using the vacuum oven were photographed using a scanning electron microscope. Figure 3.3 (right) shows a PG-infused piece of HTC. Several pores either empty or partially filled can be seen. This provided evidence that HTC foam infusions using the vacuum oven were inadequate. It is interesting to compare the photos of POCOFoam (center) and HTC (right); the number of pores in each photo illustrates the difference in density and porosity between the two foams.
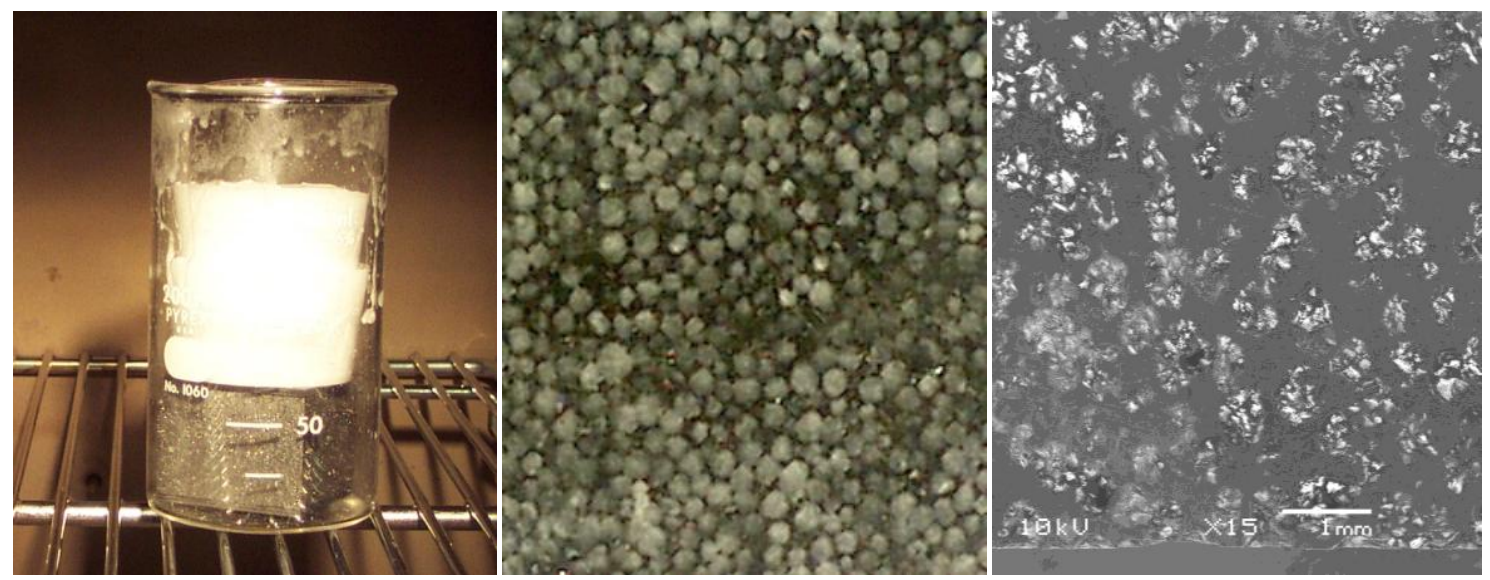

Figure 3.3: Vacuum Oven Sample/PCM Configuration (left); 10X Microphotograph of Sectioned Sample, Showing the Absence of Voids or Empty Pores (center); 15X SEM Photo of PG-Infused HTC Sample, Showing Empty Pores which Appear as Black Regions (right)

The vacuum oven method provided acceptable infusions of the POCOFoam. Two POCOFoam samples were fabricated for this project. Sample G0902.1-F, infused with docosane for early system validation tests, achieved a $98.5 \%$ fill of $27.17 \mathrm{~g}$. Sample G1003-F, having the same porosity as G0902.1-F but infused with PG, achieved only a $71.8 \%$ fill of $28.01 \mathrm{~g}$. 
To test the infuser, two samples were constructed: G1004-H and G1005-H. Both were effectively identical to G1001-H and G1002-H (the HTC experimental samples) except that the end plates were not bonded to the foam. Sample G1004-H achieved a 60\% fill of $12.32 \mathrm{~g}$ PG. It was determined that the melted PG flowed freely through the foam; much more resistance had been anticipated. The sample had an unacceptably low fill percentage, but the resulting fill was still twice what had been achieved using the vacuum oven. For G1005-H, the infusion sequence was changed in a way that allowed the melted PG to flow slowly into the infuser sight glass and solidify. The solidified PG was melted after one hour at $200^{\circ} \mathrm{C}$ and 110 psi. Once pressure was released, the PG flowed quickly from the infuser as it had for G1004-H. The solidified slug of PG provided sufficient back pressure to allow the sample to achieve a $75 \%$ fill of $15.22 \mathrm{~g}$. It was felt the fill percentage could be made higher still by more slowly melting the slug in the sight glass. This was done on sample G1002-H, which achieved a 96\% fill of $19.73 \mathrm{~g} \mathrm{PG}$. The infuser was again used to infuse sample G1001-H with tetracontane, but the wear on the device (seven hours at or above $200^{\circ} \mathrm{C}$ resulted in a failed heater element and burned piston seals) and the small quantity of tetracontane $(30 \mathrm{~g}$ ) resulted in a poor infusion: $55 \%$ fill of $9.83 \mathrm{~g}$. The modifications made to the infuser to complete the G1001-H infusion are discussed in Appendix A.

\subsection{Experimental Results}

Twelve experiments were performed on the four samples G0903-H (HTC/no PCM), G1001-H (HTC/tetracontane wax), G1002-H (HTC/PG) and G1003-F (POCOFoam/PG). The TES performance characteristics of interest in these experiments were: 1) the heater/sample interface temperature rise rate, 2) the specific latent energy storage capacity, and 3) the duty cycle exhibited by each sample.

Each sample was subjected to the same series of three tests (Table 2.2). The insulated output tests were dedicated to measuring the interface heating rates of each sample. The different fluxes would illustrate the effect of foam physical properties on the heating rates. The chilled 
output tests had two purposes. The chilled runs were used to determine the latent storage capacity of each infused sample. These runs also provided a simulated application in which the cycling performance of each sample was investigated. Sample G0903-H was included to demonstrate the difference between a block of carbon foam being heated and cooled, and a TES device storing energy in the transition enthalpy of its PCM.

\subsubsection{One-Dimensional Assumption}

An important aspect of this work is the assumption that heat flow through the sample was one-dimensional. The one-dimensional assumption greatly simplifies analysis, in that lateral losses and multi-dimensional heat flows can be neglected. To determine whether heat flow through the samples was one-dimensional, thermocouples were embedded in the center crosssectional plane of the POCOFoam/PG sample G1003-F (Figure 3.4). One thermocouple was at the center of a face, $5 \mathrm{~mm}$ deep; two other thermocouples were located at two corners of the plane, $5 \mathrm{~mm}$ from each face.

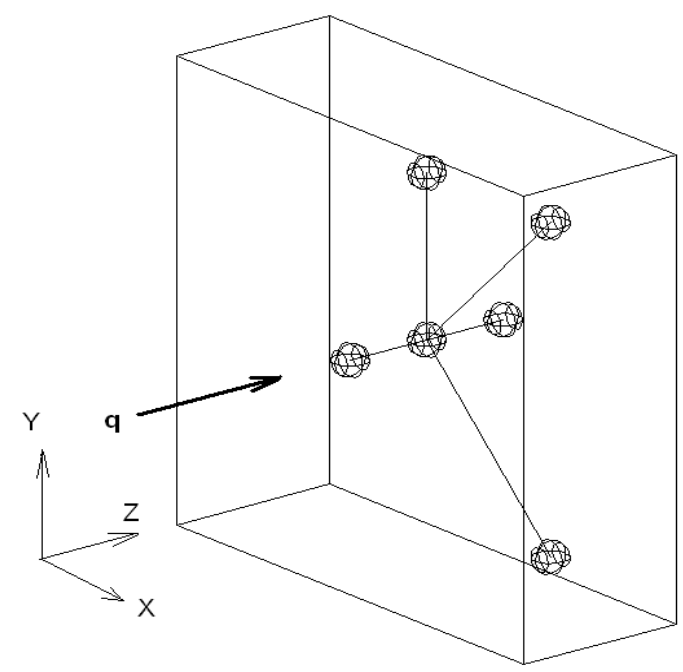

Figure 3.4: Sample G1003-F (POCOFoam/HTC) Center Plane Thermocouple Locations for One-Dimensional Heat Flow Measurement

Measurements taken during a $6.0 \mathrm{~W} / \mathrm{cm}^{2}$ insulated run are shown in Figure 3.5. The curves grouped together are the center plane thermocouple temperatures. The uppermost curve is 
the temperature profile of the axial thermocouple located $9.53 \mathrm{~mm}$ before the center plane, and the bottom curve represents the temperature profile of the axial thermocouple located $9.53 \mathrm{~mm}$ after the center plane. The center plane temperatures do show a distribution; at an elapsed time of 4190 seconds, the difference between the center thermocouple and the upper corner thermocouple was $1.0^{\circ} \mathrm{C}$. However, at that same time, the temperature difference between the center thermocouple and the axial thermocouple after the center plane was $1.7^{\circ} \mathrm{C}$; the axial thermocouples are closer to the center plane axial thermocouple than the center plane thermocouples by at least $3.17 \mathrm{~mm}$, and the thermal conductivity of the sample in the X-Y direction is approximately $40 \%$ of the conductivity in the $\mathrm{Z}$ direction. One would expect with the difference in thermal conductivities that the planar temperature differences would be larger than axial differences. Overall, the center plane thermocouple temperature differences are about $20 \%$ of the axial differences. For the purposes of this project, it was felt that the planar temperature differences could be neglected relative to the axial temperature differences.

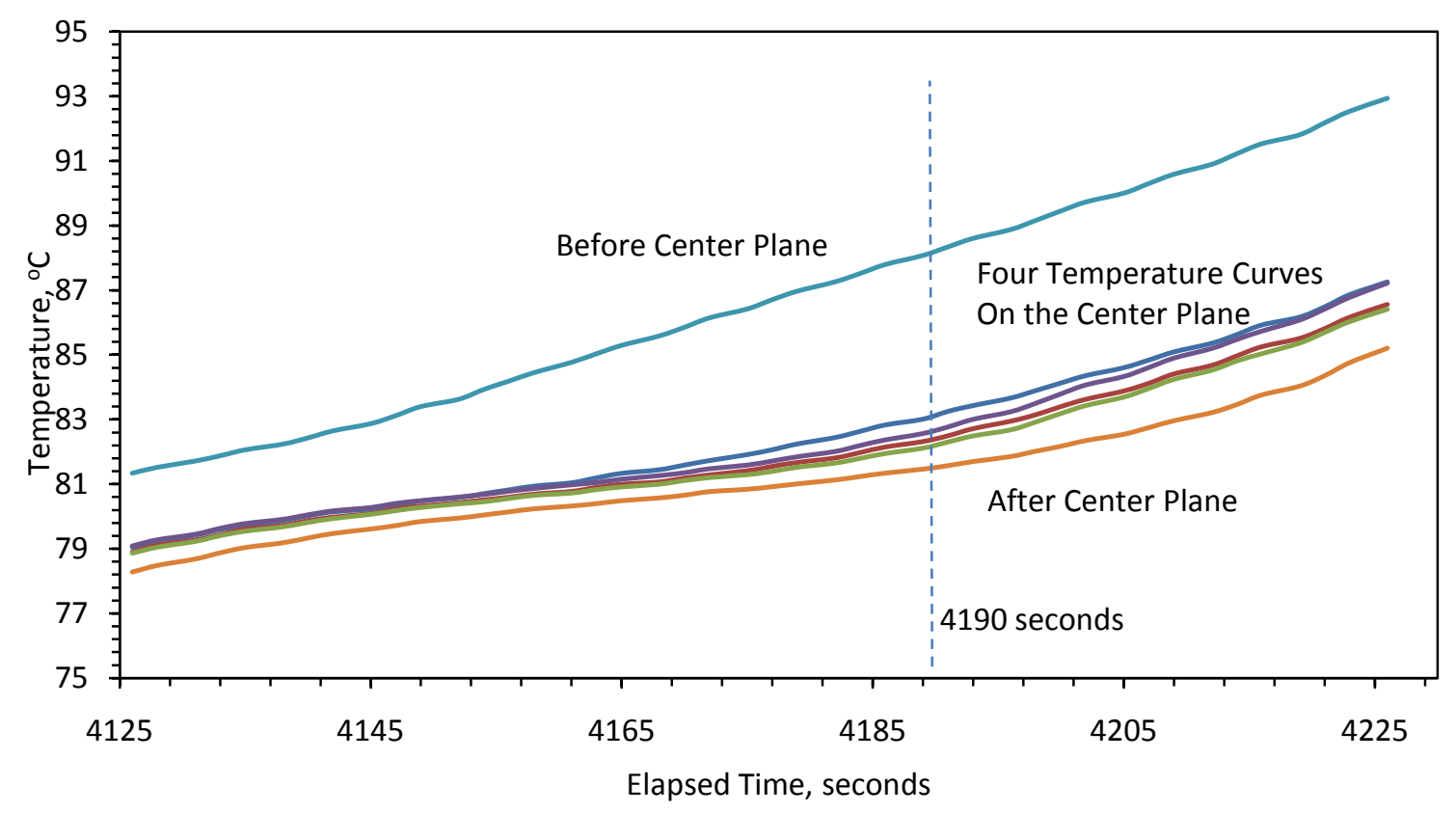

Figure 3.5: Sample G1003-F 6.0 W/cm ${ }^{2}$ Insulated Temperature History through Transition; Curves Grouped in the Middle are Temperatures on a Common Plane 


\subsubsection{Heating Rates}

The heater/sample interface heating rates for the four samples were investigated using the data from the insulated boundary experiment series. These experiments were chosen for these analyses because the temperature profiles from insulated boundary experiments more clearly show the end of transition than do experiments using a cooled output boundary; this is important for determining transition times and comparing pre-transition and post-transition heating rates (which should be similar). The temperature distributions were analyzed using the method described in Section 2.7

The effects of power and foam thermal conductivity on the interface heating rate can be best illustrated by directly comparing the temperature profiles. Figure 3.6 shows the interface temperature profiles of the last cycles of all eight runs conducted in this series; the curves are truncated to an initial temperature of $71^{\circ} \mathrm{C}$ for the sake of comparison. Each of the infused sample curves consists of three regions: pre-transition, transition, and post-transition. The right-hand peaks seen in each curve represent the temperature at which the heater power was switched off. In each of these experiments, this occurred when a sample's opposite boundary temperature reached $88^{\circ} \mathrm{C}$. Table 3.1 lists the average pre-transition, transition and post-transition rates and transition times for these runs. In Figure 3.6, the separation between the $2.3 \mathrm{~W} / \mathrm{cm}^{2}$ runs and the $6.0 \mathrm{~W} / \mathrm{m}^{2}$ runs is obvious; the higher flux causes the samples to heat more quickly. It can be seen in the figure and in Table 3.1 that the rates for all the infused samples are fairly close in a given phase region and power setting. Sample G0903-H (HTC/no PCM) heats at a faster rate than the others because it does not contain PCM. The absence of PCM is also indicated by the absence of transition regions (the curves are effectively linear). 


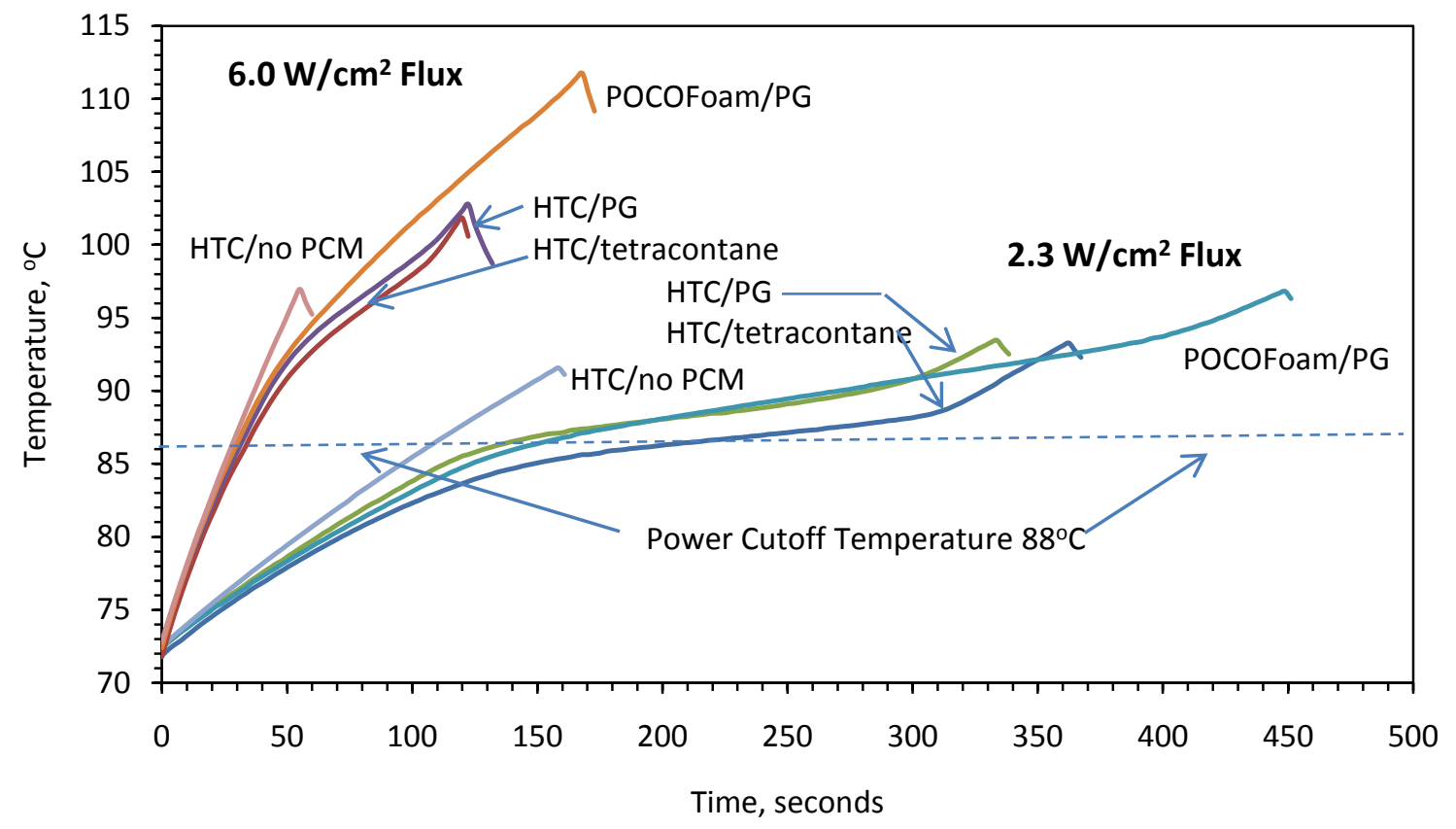

\section{Figure 3.6: Interface Heating Rates Comparison; Each Curve Is From the Last Cycle in Each Run}

The peak temperatures of sample G1003-F (POCOFoam/PG) are higher due to the lower thermal conductivity of POCOFoam, and because this sample contains approximately $50 \%$ more PCM than samples G1002-H (HTC/PG) or G1001-H (HTC/wax); the larger PCM volume also results in longer transition times (longer curves). The most notable feature of the POCOFoam/PG sample's $6.0 \mathrm{~W} / \mathrm{cm}^{2}$ curve is the peak temperature, at $111^{\circ} \mathrm{C}$. This illustrates why considering the interface heating rate is important. Sample G1003-F contains half again as much PCM as G1001$\mathrm{H}$ and $\mathrm{G} 1002-\mathrm{H}$; but under identical conditions (same power input, same power cutoff temperature), the final interface temperature is $10^{\circ} \mathrm{C}$ higher than those of the two smaller samples. Depending on the application, this difference could be outside the operating range of a connected device. Comparing the G1001-H (HTC/wax) and G1002-H (HTC/PG) curves, it appears that the two samples perform similarly regardless of the input power; the lengths, shapes and transition times of the two curves are quite similar. There are differences, though. The higher transition temperature of $\mathrm{PG}\left(83^{\circ} \mathrm{C}\right.$ vs. $81^{\circ} \mathrm{C}$ for tetracontane) is apparent in the higher temperatures of the G1002-H curves, compared to the G1001-H curves. G1002-H begins transitioning earlier than 
G1001-H at both flux levels, but it appears that G1001-H completes transition more quickly at the 6.0 W/cm ${ }^{2}$ flux than G1002-H, and more slowly at the $2.3 \mathrm{~W} / \mathrm{cm}^{2}$ flux. G1001-H demonstrates a much more gradual entrance into transition than G1002-H at both power levels. The slow entrance at the $2.3 \mathrm{~W} / \mathrm{cm}^{2}$ flux results in the long average transition time listed in Table 3.1 . Interestingly, the exit from transition appears to be more abrupt for G1001-H than G1002-H. This is likely due to the melting nature of tetracontane; referring to Figure 3.1, the DSC curve for tetracontane shows a gradual increase in transition enthalpy. Once the peak is reached, the transition enthalpy drops dramatically. This would cause abrupt heating of the sample as more energy is absorbed sensibly at that end of the sample. The DSC curve for PG (Figure 3.2) shows a more gradual increase and decrease in the transition enthalpy, which results in the more gradual heating rate changes at both ends of the transition. During transition the heating rates of G1001-H (HTC/wax) and G1002-H (HTC/PG) are nearly identical at both fluxes.

\section{Table 3.1 Heater/Sample Interface Heating Rates for the Insulated Output Experimental Series}

\begin{tabular}{cccccc}
\hline Flux $\left(\mathbf{W} / \mathbf{m}^{\mathbf{}}{ }^{\mathbf{C}}\right)$ & Sample & $\begin{array}{c}\text { Pre-Transition } \\
\left({ }^{\mathbf{0}} \mathbf{C} / \mathbf{s}\right)\end{array}$ & $\begin{array}{c}\text { Transition } \\
\left({ }^{\mathbf{}} \mathbf{C} / \mathbf{s}\right)\end{array}$ & $\begin{array}{c}\text { Post-Transition } \\
\left({ }^{\mathbf{}} \mathbf{C} / \mathbf{s}\right)\end{array}$ & $\begin{array}{c}\text { Transition Time } \\
(\mathbf{s})\end{array}$ \\
\hline 2.3 & HTC/no PCM & 0.152 & 0.112 & 0.098 & 48 \\
2.3 & HTC/wax & 0.119 & 0.037 & 0.089 & 255 \\
2.3 & HTC/PG & 0.114 & 0.036 & 0.087 & 204 \\
2.3 & PF/PG & 0.102 & 0.030 & 0.069 & 329 \\
\hline 6 & HTC/no PCM & 0.530 & 0.412 & 0.354 & 18 \\
6 & HTC/wax & 0.447 & 0.180 & 0.244 & 73 \\
6 & HTC/PG & 0.475 & 0.176 & 0.190 & 72 \\
6 & PF/PG & 0.483 & 0.195 & 0.164 & 138 \\
\hline
\end{tabular}

Table 3.2 lists the heater/sample interface transition heating rates relative to the samples G0903-H (HTC/no PCM) and G1002-H (the sample of interest; HTC/PG). Comparing the three infused samples to the empty sample G0903-H (column 4) shows that during transition, the interface heating rate of each is dramatically reduced by the PCM in transition, which is expected. 
The higher heating rate of the POCOFoam/PG sample G1003-F (column 5) is an indication of the lower thermal conductivity of the POCOFoam, compared to HTC; the higher flux magnifies the difference. The heating rates of G1001-H (HTC/wax) and G1002-H (HTC/PG) can be considered identical at both fluxes. Based on these results, it appears that the heating rates are strongly influenced by the type of foam used, and not much at all by the type or amount of PCM.

\section{Table 3.2 Heater/Sample Interface Heating Rates through Transition, Relative to the HTC/no PCM (G0903-H) and HTC/PG (G1002-H) Samples}

\begin{tabular}{ccccc}
\hline Flux $\left(\mathbf{W} / \mathbf{m}^{\mathbf{0}} \mathbf{C}\right)$ & Sample & Rate $\left({ }^{\mathbf{0}} \mathbf{C} / \mathbf{s e c o n d}\right)$ & \% HTC/no PCM Rate & \% HTC/PG Rate \\
\hline 2.3 & HTC/no PCM & 0.112 & --- & --- \\
2.3 & HTC/wax & 0.037 & 32.975 & 102.743 \\
2.3 & HTC/PG & 0.036 & 32.094 & --- \\
2.3 & PF/PG & 0.030 & 26.799 & 108.222 \\
\hline 6 & HTC/no PCM & 0.406 & --- & --- \\
6 & HTC/wax & 0.180 & 44.335 & 102.273 \\
6 & HTC/PG & 0.176 & 43.350 & --- \\
6 & PF/PG & 0.195 & 48.031 & 110.795 \\
\hline
\end{tabular}

The samples' internal heating rates and temperature profiles also illustrate interesting phenomena. Figure 3.7 shows a typical sample and interface temperature profile. A prominent feature of the plot is the temperature difference between the curve " $0 \mathrm{~mm}$ " (the interface temperature) and the curve " $2.21 \mathrm{~mm}$ " (the sample heated-end temperature). In the center of the transition region, there is a $4.6^{\circ} \mathrm{C}$ difference; this difference is due to the thermal resistances between the interface and the sample heated end. Between these two points there are actually seven sources of thermal resistance: thermal grease applied between the focus block and the holder end cap; the end cap; thermal grease between the end cap and the indium foil TIM; the TIM; the interface between the TIM and the sample end plate; the end plate; and the adhesive bond line between the end plate and the foam. The largest of these resistances is the end plate, with a thermal conductivity of $29 \mathrm{~W} / \mathrm{m}{ }^{\circ} \mathrm{C}$, resulting in a thermal resistance of $6.6^{*} 10^{-5}{ }^{\circ} \mathrm{C} / \mathrm{W}$. It is assumed that the thermal grease layers are consistent in thickness, at $0.05 \mathrm{~mm}$. A significant 
temperature difference can also be seen between the two curves 2.21 and $8.56 \mathrm{~mm}$ from the heated end and the lower three curves. This difference is believed to be caused by the density gradient in the foam. It was seen during the foam density investigation that the HTC foam has a density gradient. The lower three sample curves are very close together and experience transition at the same time, indicating that this region of the sample contains high-density foam, and relatively little PCM.

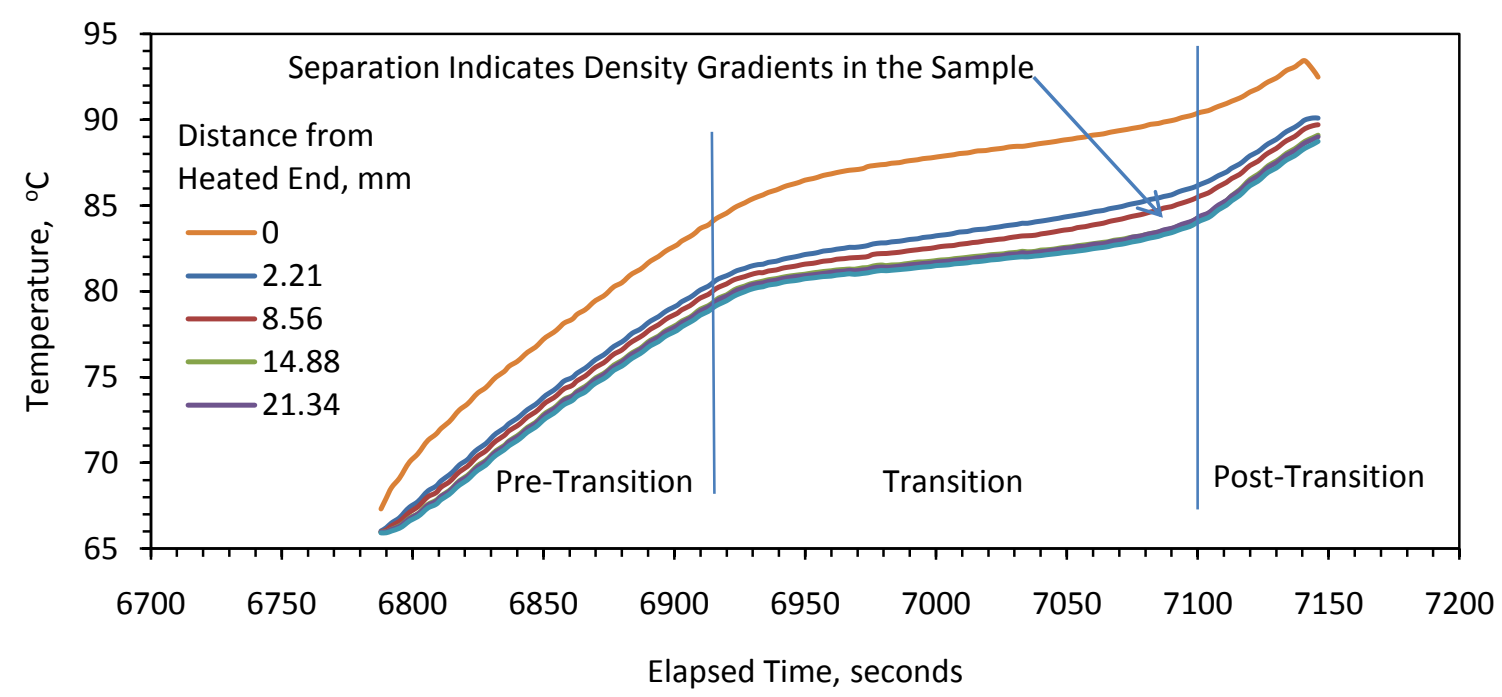

Figure 3.7: Sample and Interface Temperature Profiles for G1002-H, $2.3 \mathrm{~W} / \mathrm{cm}^{2}$ Insulated, Cycle 4

From the interface heating rate analysis presented, it can be concluded that the interface heating rate of a TES is solely a function of the thermal conductivity of the foam. A designer developing a TES where the device being cooled must be kept within a narrow range should consider using a conduction enhancer with the highest practical thermal conductivity. The HTC foam used in this project exhibits the desired property.

\subsubsection{Storage Capacities}

The latent energy stored during the chilled experiments was found by considering the form of the energy balance shown in Equation 7 (Chapter 2), repeated here: 


$$
E_{\text {st, lat }}=t_{t r}\left[q_{\text {in }}-\left(q_{\text {out }}+\sum_{i} m_{i} c_{i} \frac{d T_{i}}{d t}\right)\right]
$$

The transition times $t_{t r}$ were found by applying the heating rate analysis to the chilled run data, as was done for the insulated run data. Heat in through the focus block and heat out through the cold plate stem were calculated using the one-dimensional heat conduction equation:

$$
q=\frac{k A}{L}\left(T_{1}-T_{2}\right)
$$

The thermal conductivity values used for the heater focus block and cold plate stem were 390 and $355 \mathrm{~W} / \mathrm{m}{ }^{\circ} \mathrm{C}$, respectively. These values and the validation of the one-dimensional conduction assumption were determined during the focus block conductivity investigation of Appendix B. For the physical properties required to determine the sensible storage of the samples, the reader is referred to Table 2.3.

Table 3.3 lists the results of the storage capacity analyses; the values listed are averages calculated from the results of the last three cycles in each run. Theoretical capacities are calculated using the initial PCM mass and the transition enthalpy as determined from the DSC results. Sample G0903-H is not included in this analysis because it did not contain PCM.

Table 3.3 Total and Specific Latent Storage Capacities for Infused Samples

\begin{tabular}{cccccccc}
\hline Sample & $\begin{array}{c}\text { Theoretical } \\
\text { Capacity }\end{array}$ & $\begin{array}{c}\text { Latent } \\
\text { Energy } \\
\text { Stored }\end{array}$ & $\begin{array}{c}\text { Fraction of } \\
\text { Theoretical }\end{array}$ & $\begin{array}{c}\text { Energy } \\
/ \text { Volume }\end{array}$ & $\begin{array}{c}\text { Energy } \\
\text { /Mass }\end{array}$ & $\begin{array}{c}\text { Heat } \\
\text { Power } \\
\text { /Volume }\end{array}$ & $\begin{array}{c}\text { Heat } \\
\text { Power } \\
\text { /Mass }\end{array}$ \\
\hline G1001-H & 3250 & 1859.2 & 0.57 & 56.38 & 46.42 & 0.44 & 0.36 \\
G1002-H & 3098 & 3248.8 & 1.04 & 98.34 & 66.94 & 0.49 & 0.33 \\
G1003-F & 4397 & 6390.4 & 1.46 & 123.55 & 123.86 & 0.59 & 0.59 \\
\hline
\end{tabular}

The storage value in Table 3.3 for sample G1001-H (HTC/tetracontane) is lower than the theoretical capacity due to the shifting of PCM during cycling. It was discovered that the sample 
began to exhibit diminished storage capacity during the insulated run series; Figure 3.8 shows the sample center thermocouple temperature profiles for two separate $\mathrm{G} 1001-\mathrm{H} 6.0 \mathrm{~W} / \mathrm{cm}^{2}$ runs, performed on different days. The curve labeled "Run 2" is longer and shows transition ending later, indicating the sample had more latent storage capacity during that run. Post-test inspection of G1001-H showed that the sample had actually lost $2.23 \mathrm{~g}$, and another $2.86 \mathrm{~g}$ shifted into the expansion volume surrounding the foam. The PCM in the expansion volume does not appear to have participated in phase change, based on the low calculated storage value; this left $10.38 \mathrm{~g}$ tetracontane available to store energy in the phase change. If the theoretical capacity value is recalculated using $10.38 \mathrm{~g}$ PCM, the calculated storage capacity as a fraction of theoretical capacity becomes 0.86 .

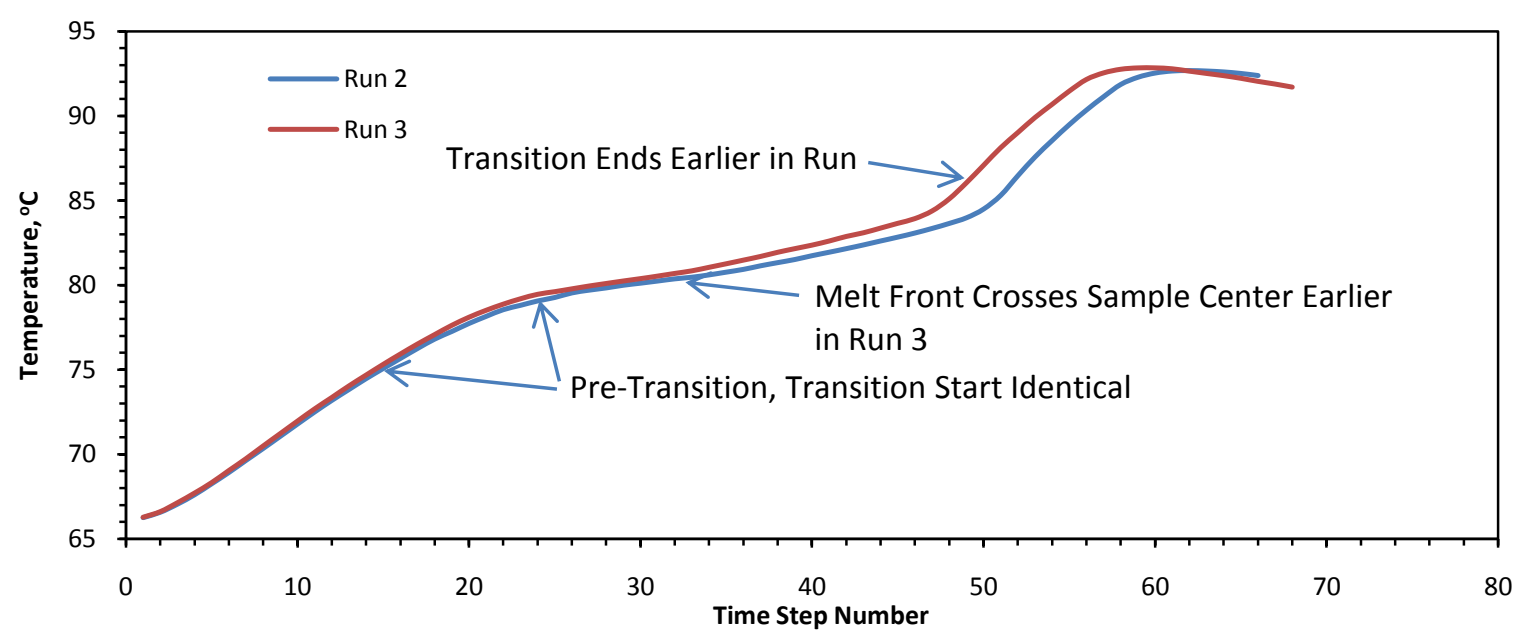

Figure 3.8: Sample G1001-H (HTC/tetracontane) Heating in Different Runs, Identical Conditions; the Storage Capacity in Run 3 Is Lower than in Run 2, Indicating Less PCM Was Available

Sample G1002-H (HTC/PG) appears to have performed as expected. The calculated latent energy stored is $4.6 \%$ above the theoretical value. The excess calculated energy stored equates to an average $0.57 \mathrm{~W}$ not included in the sensible storage terms through the transition period. 
The calculated amount of latent energy stored in sample G1003-F (POCOFoam/PG) appears to be higher than expected due to a phenomenon discovered during cycling performance analyses. During the initial per-cycle temperature distribution comparison for the G1002-H (HTC/PG) chilled run (Figure 3.9), it was found that the amount of energy stored during the first cycle was approximately $15 \%$ higher that the subsequent cycles. The sample heated and transitioned more slowly by the same amount. This phenomenon did not always appear; the percycle temperature profiles for the G1003-F (POCOFoam/PG) chilled run did not show this behavior. However, it was found that sample G0903-H (HTC/no PCM) did exhibit this behavior; clearly, the behavior was not related to latent energy storage.

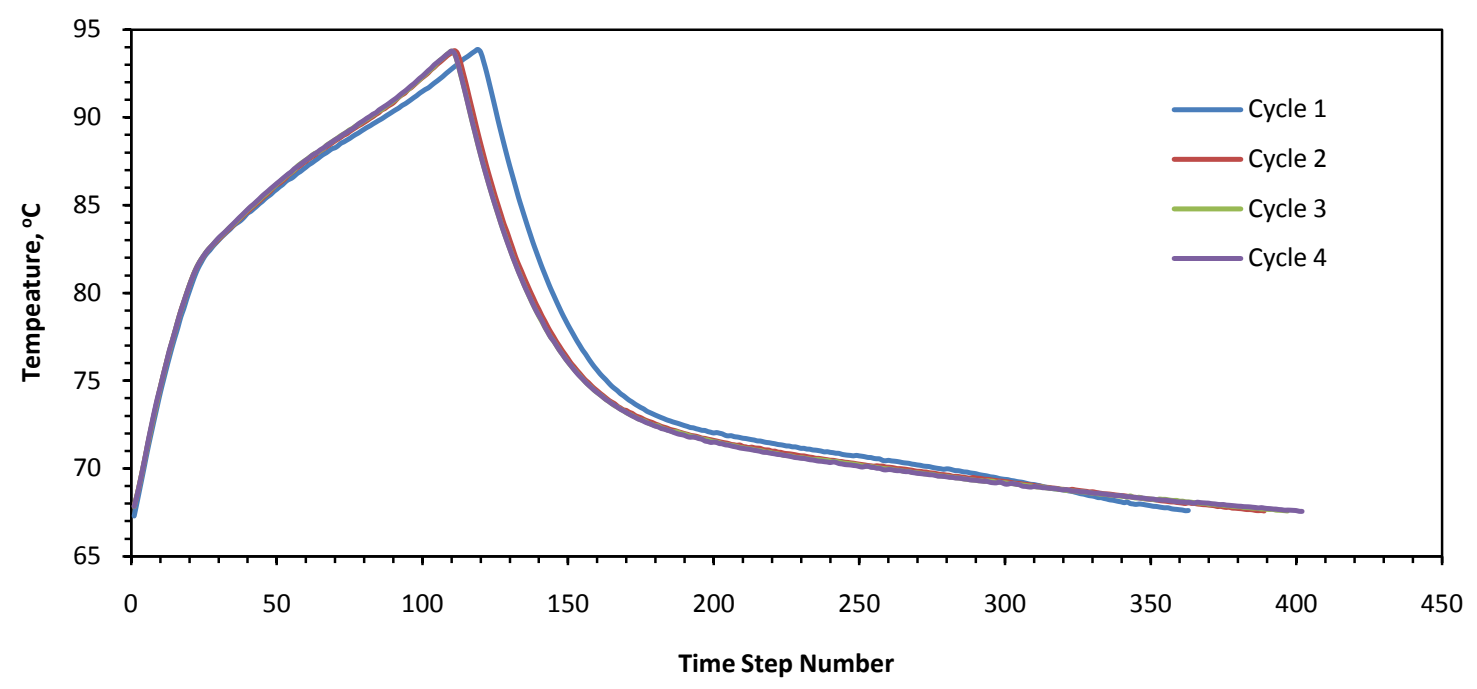

Figure 3.9: Using the Cycle Comparison for the Cold-End Thermocouple of Sample G1002H to Illustrate the Excess Energy Stored in Sample G1003-F; Cycle 1 Heating is Longer due to System Sensible Storage

By comparing various cycles of the different samples in both the insulated and the chilled runs, it became apparent that the phenomenon was due to energy being stored in a cold system. In the G1003-F POCOFoam/PG) chilled run, for each cycle the discharge period was about $1600 \mathrm{~s}$ long; in each period the cold plate removed an average of $13740 \mathrm{~J}$ from the system. The theoretical latent storage capacity of G1003-F is listed as $5377 \mathrm{~J}$, and the average energy stored sensibly in the sample through the transition period was calculated to be $2175 \mathrm{~J}$; the only other 
source of energy is the system (sample holder, insulation, and heater block) releasing heat stored sensibly before and during the transition period. The system appears to have been discharging to its pre-cycle temperatures. This phenomenon will be considered further in Section 3.2.4.

The specific energy and heat power values for the test samples are listed in columns 5 through 7 of Table 3.3. The results show that the primary factor is PCM mass. Sample G1001-H (HTC/tetracontane) should have given results similar to G1002-H (HTC/PG), but due to the PCM shift the specific energy storage is lower by the difference in fill; the ratio of specific energy capacity per unit mass of G1001-H to G1002-H divided by the ratio of G1001-H's final PCM mass to its initial mass gives a specific energy per unit mass within $4 \%$ of that demonstrated by G1002-H. The relatively high specific energy values for G1003-F (POCOFoam/PG) are indicative of the higher PCM fill due to the higher porosity of POCOFoam; a larger fraction of the sample volume is PCM.

The specific power storage values represent the average rate of latent energy storage during transition per unit volume or mass. It was felt that storage rate data should be presented, because sample G1003-F (POCOFoam/PG) was significantly larger (50\% more volume) and contained more PCM (42\% more PG) than sample G1002-H. Direct comparisons of storage energy capacities would not accurately illustrate the relative performance of the two samples.

In Table 3.3, it can be seen that Sample G1003-F (POCOFoam/PG) stores latent energy at a faster rate both per unit volume and per unit mass, due to a higher PCM fraction (more PCM transitioning at any time). G1001-H (HTC/tetracontane) and G1002-H (HTC/PG) store latent energy at approximately the same rate, but G1002-H stores faster per unit volume due to the higher fill fraction than $\mathrm{G} 1001-\mathrm{H}$, whereas $\mathrm{G} 1001-\mathrm{H}$ stores faster per unit mass because of the density difference of the two PCMs (tetracontane $0.87 \mathrm{~g} / \mathrm{cm}^{3}$, PG $1.15 \mathrm{~g} / \mathrm{cm}^{3}$ ); there is more 
tetracontane per gram than PG. This indicates that latent storage rate is a function of the volume of PCM in the TES. The storage rate does not appear to be a function of foam conductivity.

From the latent storage capacity analysis presented, it can be concluded that the latent energy storage capacity of a TES is primarily a function of the volume of PCM contained, assuming the transition enthalpy/density ratios of the PCMs are similar. A designer developing a TES where the goal is to store the maximum amount of energy in a device that uses a conduction enhancing foam should consider using a foam exhibiting the highest possible porosity, to provide a high PCM/foam volume ratio.

\subsubsection{Cycling Performance}

The primary purpose for employing TES in a thermal management system is to offer thermal protection to devices in conditions where high-rate heat rejection is either impractical or impossible. The TES system should ideally be able to discharge the stored heat quickly, so that it can be used again within a short time; it should also exhibit repeatable TES performance after many charge/discharge cycles.

It was discovered during the literature review for this project that although many researchers investigate the TES capacities of their devices, relatively few report the performance of these devices after repeated thermal cycling, or consider performance during the discharge period of the thermal cycle. Cycling work has been reported: Vrable and Yerkes (1998) report performing "over thirty" charge/discharge cycles on a hybrid TES/cold plate device; Wirtz et al. (1999) present results of a TES filled with a solid-solid PCM undergoing several charge/discharge cycles. Whether the respective systems demonstrated any performance changes due to cycling was not discussed in these past works. In this study, three TES performance characteristics related to thermal cycling were investigated: storage capacity changes due to 
cycling; the duty cycles, or charging time/discharging time ratio; and the hysteresis of the PG samples G1002-H and G1003-F due to PCM subcooling and its effect on duty cycle.

Figure 3.10 shows the input power and sample thermocouple temperature profiles during the complete G1002-H (HTC/PG) chilled run. The figure is representative of the chilled runs; each sample was subjected to four complete charge/discharge cycles, with a subsequent cycle occurring immediately after the sample opposite boundary reached the recycle temperature, which for these experiments was set at $67.5^{\circ} \mathrm{C}$. Qualitatively it can be seen that the sample cycles look similar, suggesting that repeatability is high. The curve labeled "Power" gives an estimate of the duty cycle. The discharge period (decreasing temperatures, zero power) appears to be approximately twice as long as the charging period (increasing temperatures, power near $80 \mathrm{~W}$ ); it appears the duty cycle for this sample is about $33 \%$.

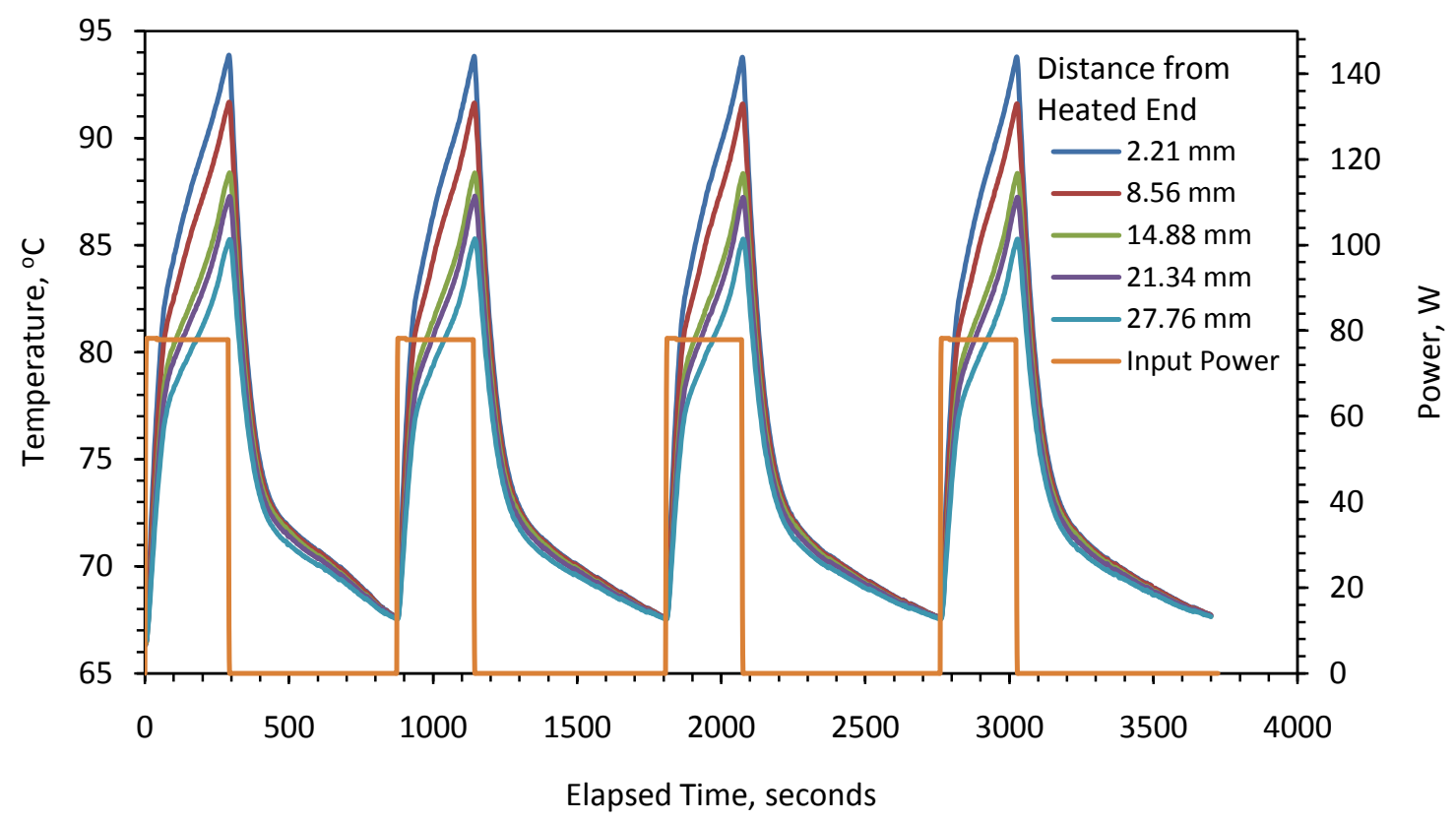

\section{Figure 3.10: Power and Sample Temperature Curves for the Complete G1002-H Chilled Run, Showing Four Charge/Discharge Cycles}

Figure 3.11 shows the sample heated-end temperature profile during the chilled run of sample G1002-H (HTC/PG) for four cycles. It appears that the sample had less storage capacity 
after the first cycle, which would indicate a loss of PCM or some other phenomenon that affected the latent storage capacity in the subsequent cycles. It was later discovered, as addressed in Section 3.2.3, that the longer first cycle was due to the cold system storing energy sensibly, causing the transition period of the first cycle to be artificially long. Further evidence of this is can be seen in the tails of the curves, during the discharge periods; the sample reached the recycle temperature approximately 100 seconds faster during the first cycle than the subsequent cycles, indicating that the system had less stored energy to release to reach the recycle temperature. Cycle 1 also shows a clear post-discharge cooling rate increase, also caused by the system having less energy to release. Cycles 3 and 4 are effectively identical, indicating that the system had reached a stable minimum stored sensible energy level by the third cycle, and that the sample was storing the same amount of energy (both latently and sensibly) in each cycle. Sample G1002-H appears to provide repeatable storage capacity; it was found that the sample stored effectively $100 \%$ its theoretical capacity (Table 3.3) even after a total of 59 cycles during the course of this work.

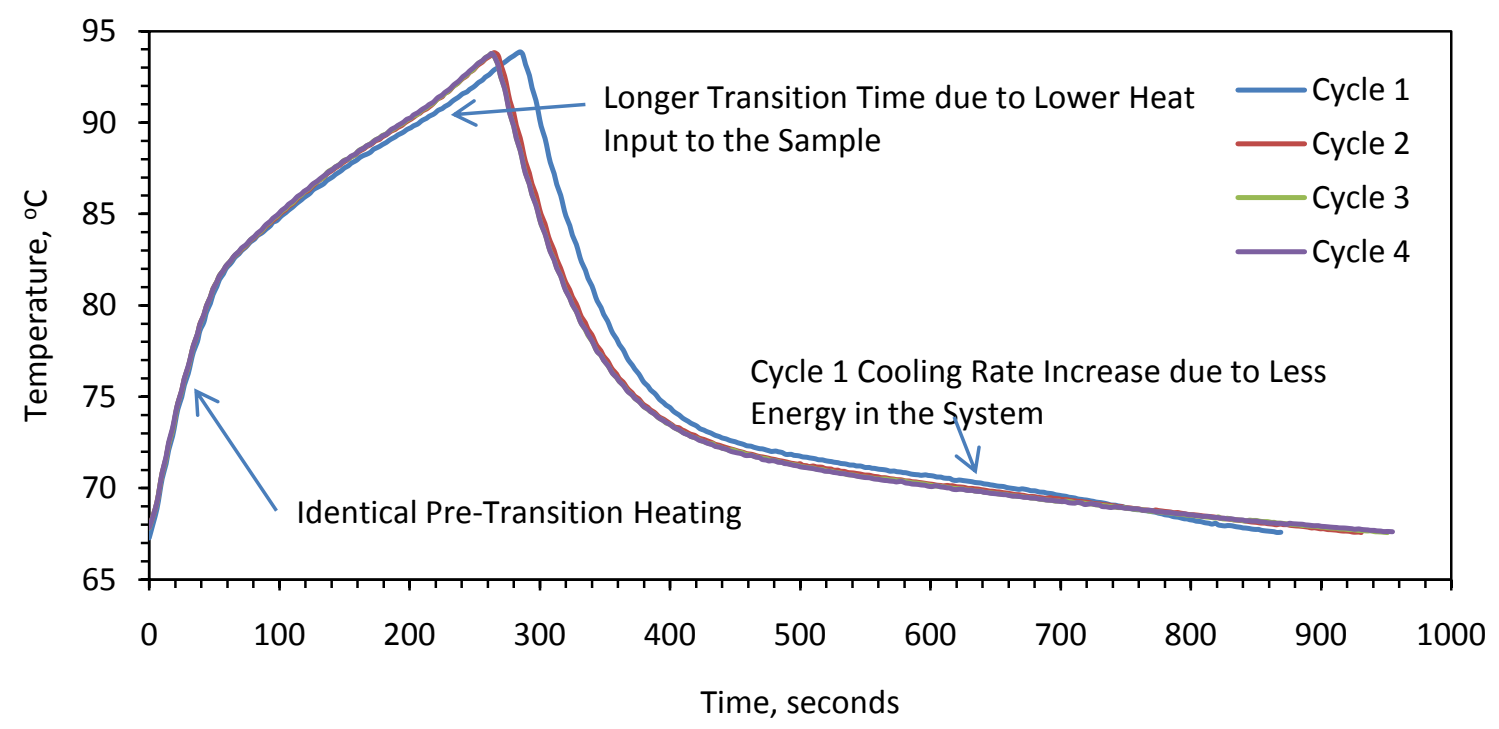

Figure 3.11: Sample G1002-H (HTC/PG) Heated-End Temperature Curves for the Four Cycles, Showing the First Cycle's Longer Transition, Faster Cooling 
Sample G1001-H (HTC/tetracontane) also exhibited repeatable storage capacity during the chilled run. However, it is known that the sample lost storage capacity at some point (Figure 3.8); the storage capacity analysis indicated after 18 cycles the sample was storing approximately half the energy it could theoretically store, assuming a constant PCM fill. It cannot be said that G1001-H provided repeatable storage capacity, based on the initial design.

A characteristic of both samples G1002-H (HTC/PG) and G1003-F (POCOFoam/PG) that can be seen in Figures 3.10-3.11 is that there is a large temperature difference between the charging temperature and the discharging temperature; PG exhibits significant subcooling. The subcooling effect was known prior to these experiments; Benson et al. (1985) discussed the dependence of the magnitude of subcooling on the heating rate, and the DSC measurements done for this effort (Figure 3.2) indicate subcooling by the displacement of the cooling curve relative to the heating curve. Figure 3.12 shows the sample temperature distribution in the fourth cycle of sample G1002-H (HTC/PG). The transition start temperature during heating is $80^{\circ} \mathrm{C}$; the transition start temperature during the cooling period is $73^{\circ} \mathrm{C}$, indicating the PCM subcools about seven degrees. The subcooling affects the overall cycling performance in that more cooling power is required to discharge a TES infused with PG, due to the difference between the operating temperature and the required sink temperature, than would be required to discharge a TES that uses a PCM that does not exhibit significant subcooling such as a paraffin wax. This is illustrated in Figure 3.13, which shows the relative cycle lengths for the two HTC samples G1001-H (tetracontane) and G1002-H (PG) during their respective chilled run fourth cycles. The discharge temperature for G1001-H (red) is approximately the same temperature as heating transition start, about $80^{\circ} \mathrm{C}$. For the respective runs, the rejection temperatures were the same $\left(68^{\circ} \mathrm{C}\right)$. 


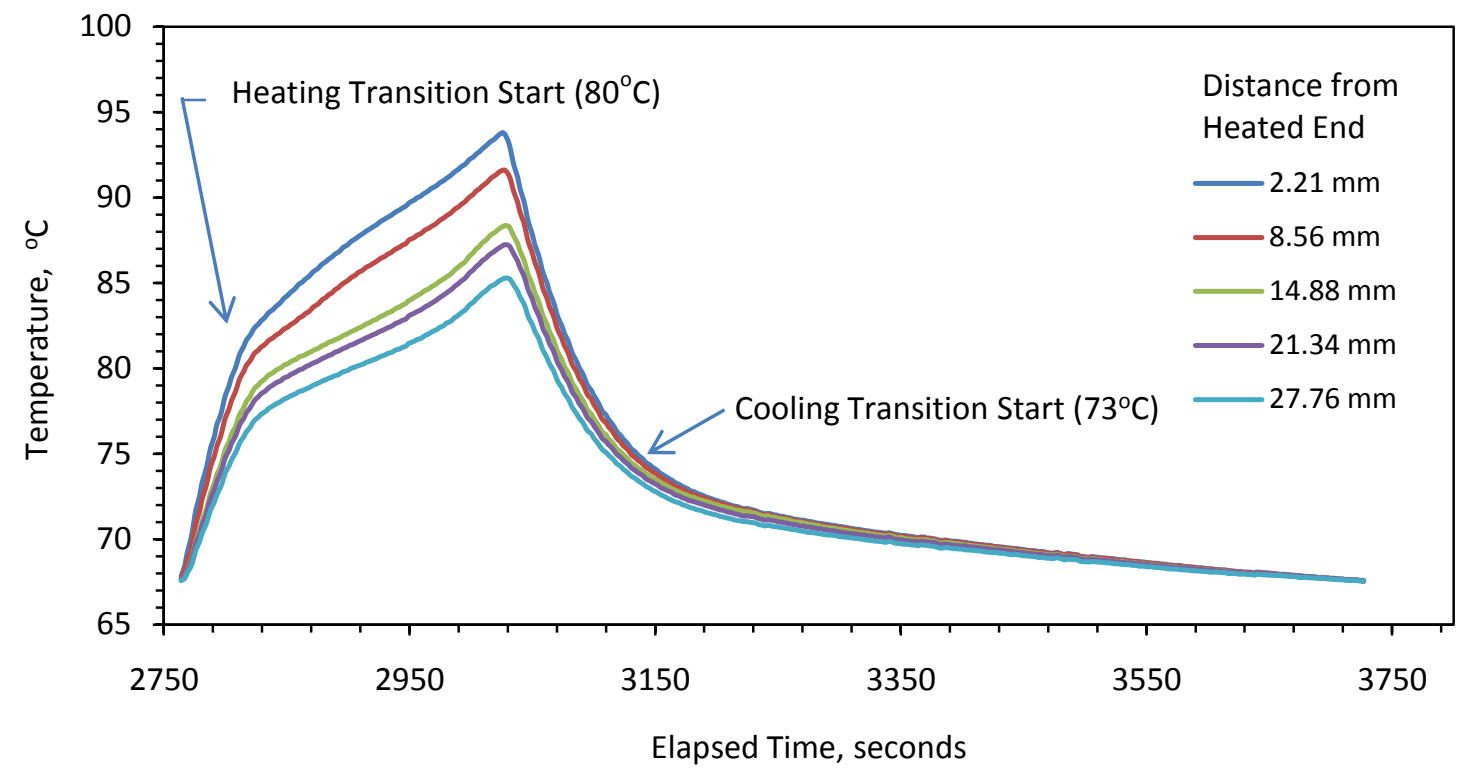

Figure 3.12: Sample G1002-H, Cycle 4: Temperature Difference Between Transition Start Temperature During Heating and Transition Start Temperature during Cooling Is $7^{\circ} \mathrm{C}$

The duty cycle of each sample, which is the ratio of TES charging time to the overall charge/discharge time, is an important performance characteristic. Duty cycle can be considered a measure of efficiency; a higher duty cycle indicates that a larger portion of the thermal cycle is spent in heat absorption, and less time in heat rejection. Ideally the duty cycle would be close to 0.5 , meaning that the TES rejects heat as quickly as it is absorbed. Table 3.4 lists the charge and discharge times, the discharge rates, and the duty cycles as averaged over the last three cycles of each sample's chilled run. The high discharge rate of G1001-H (HTC/tetracontane) is due to the large transition/rejection temperature difference; the longer charge and discharge time and lower discharge rate and duty cycle of G1003-F (POCOFoam/PG) were due to the sample containing more PCM than the other two samples. The lower thermal conductivity of the POCOFoam in G1003-F did not appear to affect the cycling performance; the only difference seen that can be attributed to the foam conductivity is the samples' internal temperature distributions, which throughout the discharge period were negligible. 
Table 3.4 Charge / Discharge Times and Rates and Duty Cycles for the Infused Samples

\begin{tabular}{ccccc}
\hline Sample & $\begin{array}{c}\text { Charging Time } \\
(\mathbf{s})\end{array}$ & $\begin{array}{c}\text { Discharging Time } \\
(\mathbf{s})\end{array}$ & $\begin{array}{c}\text { Discharge Rate } \\
\text { Duty Cycle }\end{array}$ & $\begin{array}{c}\text { Du } \\
(\boldsymbol{\%})\end{array}$ \\
\hline G1001-H & 209.44 & 503.15 & 27.61 & 26.89 \\
G1002-H & 273.98 & 701.78 & 5.63 & 30.71 \\
G1003F & 403.93 & 1794.71 & 2.88 & 18.37 \\
\hline
\end{tabular}

It can be seen in Figure 3.13 that the duty cycles for both G1001-H (HTC/tetracontane) and G1002-H (HTC/PG) are rather high. Based on the power on/off times shown in the figure, G1001-H demonstrates a duty cycle of 0.337 and G1002-H a duty cycle of 0.377 . The amount of energy that G1001-H absorbs per cycle is known to be less than was intended, and is visibly less than that stored by G1002-H. The duty cycle of each sample is artificially inflated by the high power cutoff temperature, which for these runs was five degrees higher than the end of heating transition temperature. If the excess power absorbed is subtracted, the duty cycles for $\mathrm{G} 1001-\mathrm{H}$ and G1002-H are 0.27 and 0.31 , respectively. This is reflected in the duty cycle values listed in Table 3.4. 


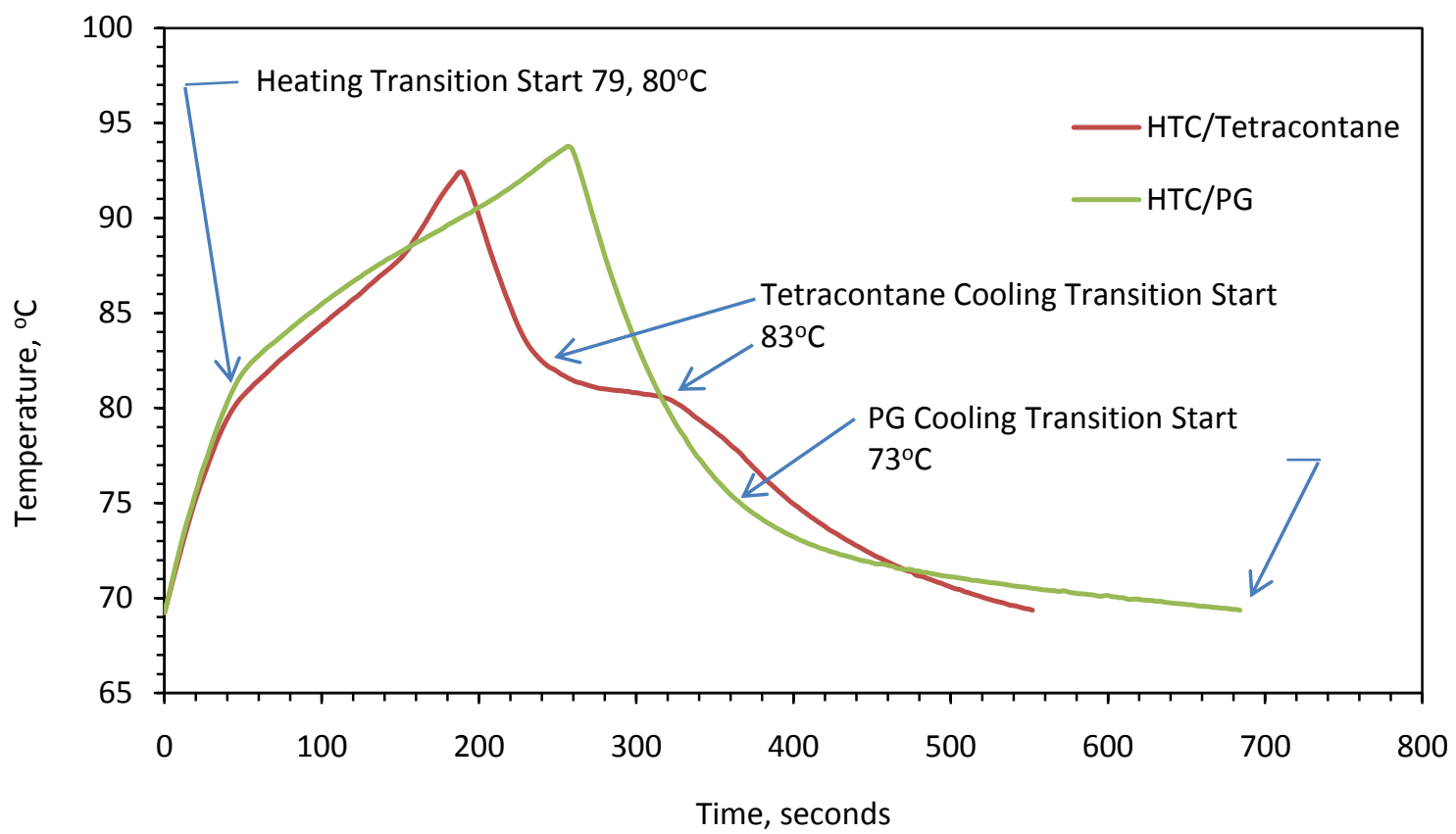

Figure 3.13: Sample Hot-End Temperature Distributions for Samples G1001-H (HTC/wax) and G1002-H (HTC/PG) During the Fourth Cycle of the Chilled Runs; G1002-H Exhibits Subcooling, G1001-H Does Not

A TES containing PG has to be cooled below the heating transition temperature to be fully discharged; the G1001-H (HTC/tetracontane) curve in Figure 3.13 shows that only about half the full cycle is spent with the PCM transitioning (melting or freezing), due to the difference between the transition and sin temperatures, but G1002-H (HTC/PG) is in transition to the end of its cycle. It could reasonably be assumed that subcooling has an adverse effect on the duty cycle. Figure 3.14 shows this is not the case. In the figure, the second cycle of G1003-F (POCOFoam/PG) and sample G0902.1-F (POCOFoam, docosane PCM) during their respective $6.0 \mathrm{~W} / \mathrm{cm}^{2}$ chilled runs can be seen to have approximately the same cycle length. Sample G1003F exhibits subcooling, as seen by the lower discharge temperature, whereas sample G0902.1-F does not exhibit subcooling; the heating transition end temperature and the cooling transition start temperature are approximately $45^{\circ} \mathrm{C}$. The primary determining factor of TES duty cycle appears to be the temperature difference between PCM transition and heat rejection; if these temperatures are close, as would be the case in a minimum-cooling application (which these experiments were 
intended to simulate) the duty cycle is quite low. The heating period of G1003-F was 374 seconds and the overall cycle was 2056 seconds, for a duty cycle of 0.184; the heating period of G0902.1F was 182 seconds with an overall cycle length of 1855 seconds, for a duty cycle of 0.098 . In this case, with samples (except for the PCM) and conditions being identical, the subcooling did not affect the duty cycle.

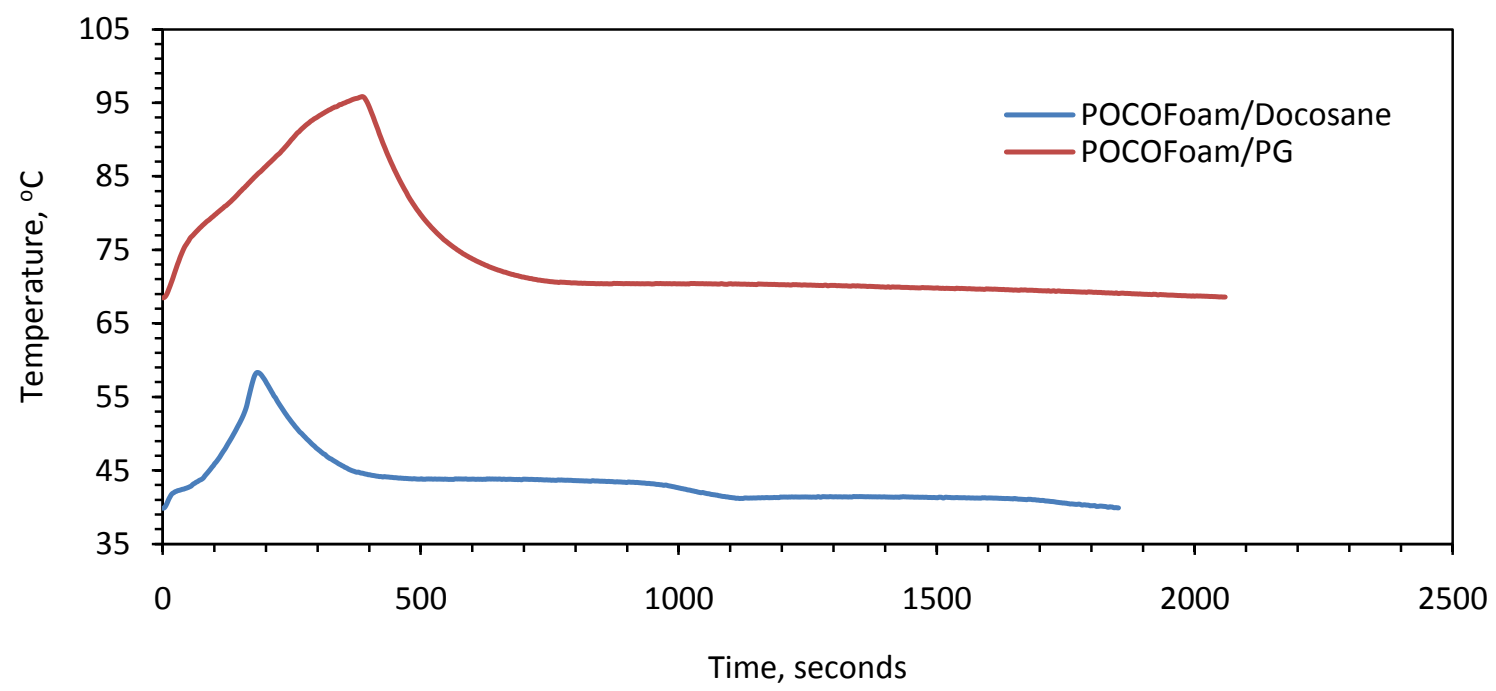

Figure 3.14: Samples G0902.1-F (POCOFoam/docosane) and G1003-F (POCOFoam/PG),
Chilled Second Cycle; Overall Cycle Times and Duty Cycles Are Similar

From the analysis presented, it can be concluded that the three TES performance characteristics related to cycling depend most strongly on the volume of PCM contained and the temperature difference between the discharging device and the sink. A designer developing a TES where the goal is to maximize the storage capacity repeatability of the device should consider avoiding PCMs that can leak from the device or shift away from the thermal path. If the goal is to optimize the duty cycle of the device, the designer should consider choosing a PCM with a high energy density. PCMs with low energy density require longer discharge times due to the volume of PCM required for a desired storage capacity. It is assumed the TES discharge/sink temperature difference is small; otherwise a TES would not be required. Minimizing the cooling power therefore requires a PCM that does not exhibit significant subcooling. 


\section{Chapter 4: Conclusion}

\subsection{Summary}

The primary goal of this project was to construct and test a TES device that uses the solid-solid PCM PG and POCO HTC carbon foam conduction enhancement. This device and others similarly constructed but using a different PCM or foam (or both) were subjected to thermal conditions intended to illustrate the TES performance unique to the HTC/PG device, with respect to its interface heating rate, latent energy storage capacity and cycling behaviors. Twelve experiments were conducted on the four samples G0903-H (HTC/no PCM), G1001-H (HTC/tetracontane), G1003-F (POCOFoam/PG), and G1002-H (HTC/PG) for different boundary conditions. Additionally, results of tests conducted on a fifth sample G0902.1-F (POCOFoam/docosane) were used to illustrate cycling performance.

\subsection{Conclusion}

Based on the experiments performed in this project, it appears that the HTC/PG combination does make for an effective TES device. Sample G1002-H demonstrated a storage capacity that was $100 \%$ of the theoretical capacity, and it retained $100 \%$ of that capacity after 59 separate thermal cycles under various conditions, many of those cycles contiguous. During transition it demonstrated a $63 \%$ reduction in the heater/TES interface heating rate at $6 \mathrm{~W} / \mathrm{cm}^{2}$ input flux, providing a longer operating period before the PCM had fully transitioned. It demonstrated a $31 \%$ duty cycle under minimal cooling conditions (the sample discharge end 
temperature was equal to the sink temperature). Furthermore, the duty cycle of the sample was not adversely affected by the PG subcooling. It was initially assumed that the lower discharge temperature would result in longer discharge times; when the amount of energy stored sensibly during heating is included in the total storage capacity, the duty cycles for the PG samples were comparable to the wax samples under minimal cooling conditions.

It was difficult to directly compare the results of the HTC/PG sample experiments with the HTC/tetracontane sample experiments because during the insulated condition test series the HTC/tetracontane sample underwent a PCM shift (wax extruded from the foam into the surrounding expansion space) which effectively halved the latent energy storage capacity of the sample. That sample had been designed and built to have a capacity comparable to the HTC/PG sample. The motivation for exploring TES using a solid-solid PCM was to eliminate PCM leakage and the resultant loss of storage capacity. In that regard, the HTC/tetracontane sample G1001-H met the objective of demonstrating storage capacity retention due to PCM retention. Despite the loss in storage capacity, G1001-H and G1002-H (HTC/PG) provided the same interface temperature regulation rate and the two samples exhibited comparable duty cycles.

Direct comparison of the performance of the HTC/PG sample to the POCOFoam/PG sample G1003-F was difficult because the POCOFoam/PG sample was a different size. This forced the consideration of specific performance characteristics (per unit mass or volume) such as specific discharge rates or heating rates. The POCOFoam/PG sample did consistently illustrate the performance aspects where PCM volume or mass was the driving factor, such as total storage capacity and duty cycle. In hindsight, sample G1003-F should have been fabricated to the same physical dimensions as the HTC samples. The performance differences due to the lower thermal conductivity and higher porosity of POCOFoam would still have been apparent. 
Sample G0903-H deserves recognition. This sample was originally abandoned due to a faulty infusion (docosane in HTC, using the vacuum oven), and no there were no plans to test it; but during the chilled experiment series, it became clear that a sample that did not exhibit latent energy storage would be useful in illustrating the performance characteristics that were based on sensible storage, such as sensible heating and cooling rates through the transition temperatures. It was the results of the $\mathrm{G} 0903-\mathrm{H} 6.0 \mathrm{~W} / \mathrm{cm}^{2}$ insulated run that led to the discovery of the cause of the cold-system storage capacity distortion.

Assuming the results of the experiments have illustrative value, the performance characteristics of the three infused samples have been ranked and listed in Table 4.1. The performance of each sample is given a rank from 1 (best) to 3 (worst); the sample with the lowest score can be considered to exhibit the optimum (based on this limited pool) combination of performance characteristics.

Table 4.1 TES Performance Merit Matrix

\begin{tabular}{cccc}
\hline $\begin{array}{c}\text { Performance } \\
\text { Characteristic }\end{array}$ & $\begin{array}{c}\text { G1001-H } \\
\text { HTC/wax }\end{array}$ & $\begin{array}{c}\text { G1002-H } \\
\text { HTC/PG }\end{array}$ & $\begin{array}{c}\text { G1003-F } \\
\text { POCOFoam/PG }\end{array}$ \\
\hline Heating Rate & 1 & 1 & 3 \\
Latent Storage & 3 & 2 & 1 \\
Duty Cycle & 1 & 2 & 3 \\
Repeatability & 3 & 1 & 1 \\
\hline Total & 8 & 6 & 8 \\
\hline
\end{tabular}

In some of the categories, there was no clear best performer. In these categories the better performing samples were given equal ranks. For the interface heating rate, G1001-H (HTC/tetracontane) and G1002-H (HTC/PG) performed equally well, because the determining factor was the thermal conductivity of the foam. Sample G1001-H clearly demonstrated the worst storage performance; its calculated capacity was about half the expected value; sample G1003-F (POCOFoam/PG) had the best performance in the storage capacity category simply because it contained more PCM than the other samples. G1001-H demonstrated the highest duty cycle, but 
only because it did not store as much energy and therefore discharged more quickly. Samples G1002-H and G1003-F both demonstrated near-perfect repeatability, because PG does not leak. The result is that sample G1002-H is deemed to have exhibited the best overall performance, only because it did not exhibit the worst performance in any category. Based on the results of this project, it can be concluded that for systems that operate in the temperature range of $65^{\circ} \mathrm{C}$ to $100^{\circ} \mathrm{C}$ with minimal active cooling, the HTC/PG combination demonstrates TES performance superior to more traditional carbon foam/wax TES devices.

\subsection{Recommendations for Further Study}

This report discussed the results of TES performance experiments performed on the HTC/PG and other samples fabricated for this study. This report does not represent an exhaustive exploration of the capabilities of solid-solid PCMs infused in carbon foams; other topics can certainly be investigated. Some possible candidate topics that have been considered during this effort are listed below.

\subsubsection{Environmental Stability}

Applying TES to aerospace systems could require that they operate in extreme environments. PG has two known characteristics related to environmental stability: PG has a high vapor pressure at the transition temperature, which could affect extreme altitude TES performance; and PG is hygroscopic, which could affect TES performance in high-humidity environments. TES performance under these conditions should be explored before PG can be considered a reasonable replacement for paraffins.

\subsubsection{Temperature Ranges}

Benson et al. (1985) showed that PG can be alloyed with other tetrahedral polyalcohols such as pentaerythritol (PE) and neopentylglycol (NPG), with the result of changing the bulk transition temperature and enthalpy. It is assumed that the $65^{\circ} \mathrm{C}-100^{\circ} \mathrm{C}$ range demonstrated in 
this work represents only a small portion of potential TES operating temperatures. Alloys of PGPE have the capability of a range from $65^{\circ} \mathrm{C}$ to $200^{\circ} \mathrm{C}$, depending on the mixture percentages (Barrio et al. (1988)). The TES performance of these alloys infused in carbon foams could be investigated.

\subsubsection{Modeling and Simulation}

The heat transfer physics at the foam/PCM interface is rather complicated; if the PCM exhibits solid-solid phase change, the physics is even more interesting. The heat transfer from foam to PCM and within the PCM itself could be explored numerically, and perhaps a standard model for solid-solid TES parametric studies could be developed. Work has begun in this field; data generated for this report have been applied to existing heat transfer models for model validation. This work is expected to continue.

\subsubsection{HTC/PG TES Prototypes}

Wirtz et al. (2003) showed that the PCM-infused carbon foam/carbon plate combination can be used as a structural material that incorporates TES. This behavior could be exploited to develop prototype structures that use a solid-solid PCM for TES, such as equipment racks, power electronics housings or even motor frames, for example. Operational prototypes could be developed and tested. 


\section{References}

Bach, P.W., Haige, W.G. "Heat Storage and Transformation," paper ECN-RX-01-036, presented at the ZAE-Symposium 2001, 3-5 October 2001.

Barrio, M., Font, J., Muntasell, J., Navarro, J., Tamarit, J.L. “Applicability for Heat Storage of Binary Systems of Neopentylglycol, Pentaglycerine and Pentaerythritol: A Comparative Analysis," Solar Energy Materials 18, 1988.

Barrio, M., Font, J., Lopez, D.O., Muntasell, J., Tamarit, J.L1. “Thermal Expansion Tensors of Pentaerythritol (PE) and Pentaglycerin (PG) and Compositional Deformation Tensor of $\mathrm{PG}_{1-\mathrm{x}} \mathrm{PE}_{\mathrm{x}}$ Molecular Alloys," J. Appl. Cryst. 27, pp. 527-531, 1994.

Bauer, C., Wirtz, R.A. "Thermal Characteristics of a Compact, Passive Thermal Energy Storage Device," Paper 2-e-2-1, ASME International Mechanical Engineering Conference and Exhibit, Orlando, FL, November 2000.

Benson, D.K., Burrows, R.W., Webb, J.D. "Solid-State Transitions in Pentaerythritol and Related Polyhydric Alcohols," Solar Energy Materials 13, pp. 133-152, 1986.

Benson, D.K., Webb, J.D., Burrows, R.W., McFadden, J.D.O., Christensen, C. Materials Research for Passive Solar Systems:Solid-State Phase Change Materials, Solar Energy Research Institute, SERI/TR-255-1828, 1985.

Chandra, D., Ding, W., Lynch, R.A., Tomlinson, J.J. "Phase Transitions in 'Plastic Crystals'," Journal of the Less-Common Metals 168, pp. 159-167, 1991.

Farid, M.M., Khudhair, A.M., Razack, S.A.K., Al-Hallaj, S. "A review on phase change energy storage: materials and applications," Energy Conversion \& Management 45, pp. 15971615, 2004.

Gallego, N., Klett, J.W. "Carbon Foams for Thermal Management," Carbon 41, pp. 1461-1466, 2003.

Hale, D.V., Hoover, M.J., O’Neill, M.J. Phase Change Materials Handbook, NASA C-61363, pp. 5-12-5-39, 1971. 
Harris, R., Leland, Q., Du, J., Chow, L.C. "Characterization of Paraffin-Graphite Foam and Paraffin-Aluminum Foam Thermal Energy Storage Systems," AIAA-2006-2132, $9^{\text {th }}$ AIAA/ASME Joint Thermophysics and Heat Transfer Conference, 5 June 2006.

Heinz, A., Streicher, W. "Application of Phase Change Materials and PCM-Slurries for Thermal Energy Storage," Institute of Thermal Engineering, Graz University, 2006.

Incropera, F.P., DeWitt, D.P. Introduction to Heat Transfer, John Wiley \& Sons, pp. 43-50, 1985.

Johnson, D.J., Clement, S., Ervin, J.S., Leland, Q. "Studies of Thermally Conductive Foams Infused with Paraffins for Thermal Storage Applications," $5^{\text {th }}$ Dayton Engineering Science Symposium, Dayton OH, 26 October 2009.

Lourdin, D., Roux, A.H., Grolier, J.P.E., Buisine, J.M. "Thermobarometric and differential scanning calorimetric study of the polymorphism of some even and odd paraffins $\left(\mathrm{C}_{26}\right.$, $\left.\mathrm{C}_{27}, \mathrm{C}_{40}, \mathrm{C}_{60}\right)$," Thermochimica Acta 204, pp. 99-110, 1992.

Mesalhy, O., Lafdi, K., Elgafy, A. "Carbon foam matrices saturated with PCM for thermal protection purposes," Carbon 44, 2006.

Moore, D.S., McCabe, Introduction to the Practice of Statistics, $5^{\text {th }}$ Ed., W.H. Freeman and Company, New York, pp. 450-454, 2006.

Morea, S.F., “'The Lunar Roving Vehicle'--- A Historical Perspective," Lunar and Planetary Institute Contribution 652, $2^{\text {nd }}$ Conference on Lunar Bases and Space Activities of the $21^{\text {st }}$ Century, Houston, TX, 5-7 April, 1988.

Py, X., Olives, R., Mauran, S. "Paraffin/Porous-Graphite-Matrix Composite as a High and Constant Power Thermal Storage Material," Intl. J. Heat and Mass Transfer 44, pp. 2727-2737, 2001.

Rudman, R. "Structural Relationships Between the Orientationally Disordered (Plastic) and Ordered Phases of Tetrahedral Molecules," Solid State Communications Vol. 29, pp.785787, 1979.

Vrable, D.L, Yerkes, K.L. "A Thermal Management Concept for More Electric Aircraft Power Systems Applications," SAE Technical Paper Series 981289, 1998.

White, M.A. "Heat storage systems", in AccessScience@McGraw-Hill, http://www.accessscience.com, DOI 10.1036/1097-8542.YB020415, 25 March, 2002.

Wierschke, K. W. "Thermal Characteristics of Pitch Based Carbon Foam and Phase Change Materials," M.S. Thesis, Air Force Institute of Technology, Wright-Patterson AFB, OH, 2005 .

Wilmet, F., Ribet, M., Bernier, P., Girault, Y., Elegant, L. " ${ }^{13}$ C NMR Investigation of the SolidPlastic Phase Transitions in Polyols and their Mixtures," Solid State Communications

Vol. 76, pp. 621-626, 1990. 
Wirtz, R., Fuchs, A., Narla, V., Shen, Y., Zhao, T., Jiang, Y. “A Multi-Functional Graphite/Epoxy-Based Thermal Energy Storage Composite for Temperature Control of Sensors and Electronics," AIAA-2003-513, 41 ${ }^{\text {st }}$ Aerospace Sciences Meeting and Exhibit, Reno, NV, 6-9 January, 2003.

Wirtz, R. A., Zheng, N., and Chandra, D., "Thermal Management Using Dry Phase Change Materials," Proceedings IEEE Semiconductor Thermal Measurement and Management Symposium, pp. 74-82, 1999.

Xiao, M., Feng, B., Gong, K. “Thermal Performance of a High-Conductive Shape-Stabilized Thermal Storage Material,” Solar Energy Materials \& Solar Cells 69, pp. 293-296, 2001.

Yang, R., Xi, H., Zhang, Y. "Preparation, physical property and thermal physical property of phase change microcapsule slurry and phase change emulsion," Solar Energy Materials \& Solar Cells 80, pp. 405-416, 2003

Yu, Q., Straatman, A.G., Thompson, B.E. "Carbon-foam finned tubes in air-water heat exchangers," Applied Thermal Engineering 26, pp. 131-143, 2006.

Zalba, B., Marin, J.M., Cabeza, L.F. "Review on thermal energy storage with phase change: materials, heat transfer analysis and applications," Applied Thermal Engineering 23, pp. 251-283, 2003.

Zheng, N., Wirtz, R.A. "A Hybrid Thermal Energy Storage Device, Part 1: Design Methodology," J. Electronic Packaging, Vol. 126, pp. 1-7, 2004. 


\section{Appendix A: Infuser Modifications}

\section{A.1 Modification 1: Tetracontane Reinfusion}

The infuser, described in Chapter 2, was also used to infuse sample G1001-H with tetracontane. This infusion was more difficult than the PG infusions, because while the PG infusions had large quantities of PCM available, there was only $30 \mathrm{~g}$ tetracontane available for G1001-H. The minimum quantity of tetracontane required for G1001-H infusion was $23 \mathrm{~g}$, based on the foam porosity and the lost volume due to the infuser head. Excess PCM would likely be trapped in the infuser, and would not be forced out as had the PG infusions.

The G1001-H infusion was performed after the two PG infusion tests and the G1002-H PG infusion. At that point, the infuser had operated for about seven hours at above $200^{\circ} \mathrm{C}$ and 100 psi; the piston seals were burned (Figure A.1) and one heater element had failed. The operator was unaware of these problems.

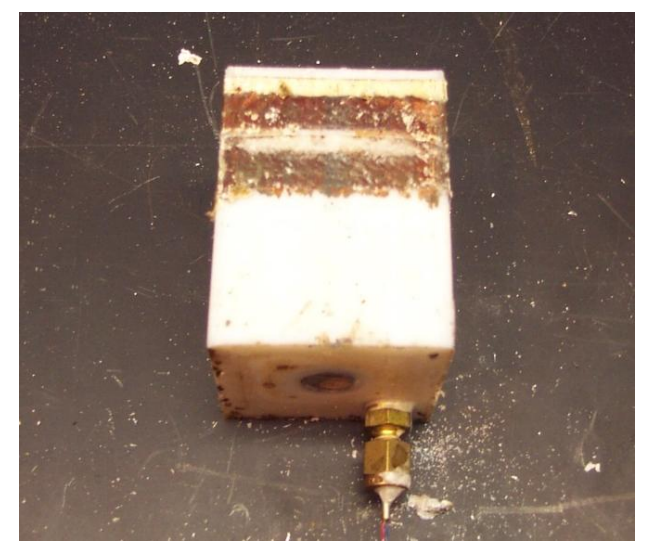

Figure A.1: Infuser Piston Showing Burned Packing Seals, after Seven Hours Above $200^{\circ} \mathrm{C}$ 
During the HTC/tetracontane infusion, the piston seals began to leak; once the PCM had completely melted, the piston completed its cycle and more than half the PCM leaked around the piston. Approximately eight grams were lost; $9.83 \mathrm{~g}$ had been infused (for a $63 \%$ fill), and $11.8 \mathrm{~g}$ were recovered. This quantity was insufficient to complete the infusion of G1001-H using the infuser as it was configured.

The method used to complete the infusion was a modification of the pressure infiltration developed by the foam manufacturer. The sample would be immersed in melted PCM, and pressurized air applied to force the PCM into the foam. The infuser was modified so that the piston was no longer used to force PCM through the foam; instead of filling the chamber with PCM, the sample and the remaining tetracontane were placed in a copper sleeve that was placed in the chamber. Figure A.2 shows the sleeve cut open and sample G1001-H after reinfusion. The infuser head was also abandoned (it was not need to hold the sample, and it would have taken unnecessary chamber volume). In its place, a plate with a tapped pressure port (Figure A.3) was sealed to the top of the chamber, and a thermocouple passed through the pressure port and contacted the sample, to replace the thermocouple lost with the infuser head. The infuser was set to infuse at $90^{\circ} \mathrm{C}$ and 110 psi for four hours after a two-hour preconditioning period at $80^{\circ} \mathrm{C}$ and 25 " vacuum to remove any remaining air trapped in the foam. Using the described modification, the G1001-H reinfusion provided a 96\% fill, with $15.48 \mathrm{~g}$ tetracontane infused.

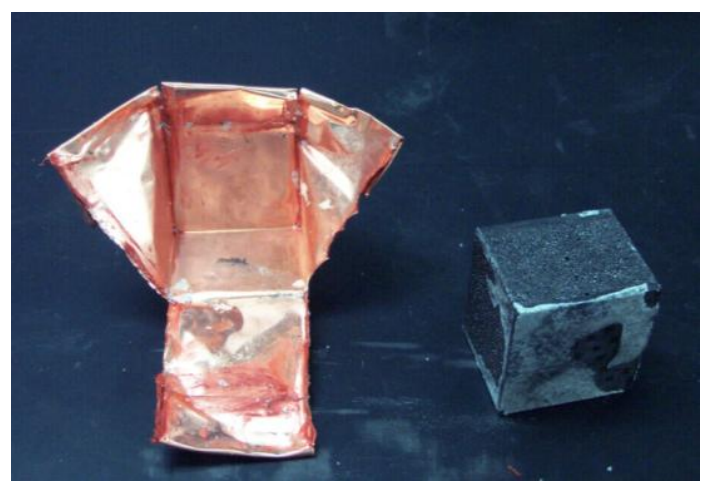

Figure A.2: Sample G1001-H and Copper Sleeve Used for Tetracontane Re-infusion; the Sleeve was Filled with the Remaining PCM and the Sample was Immersed for Infusion 


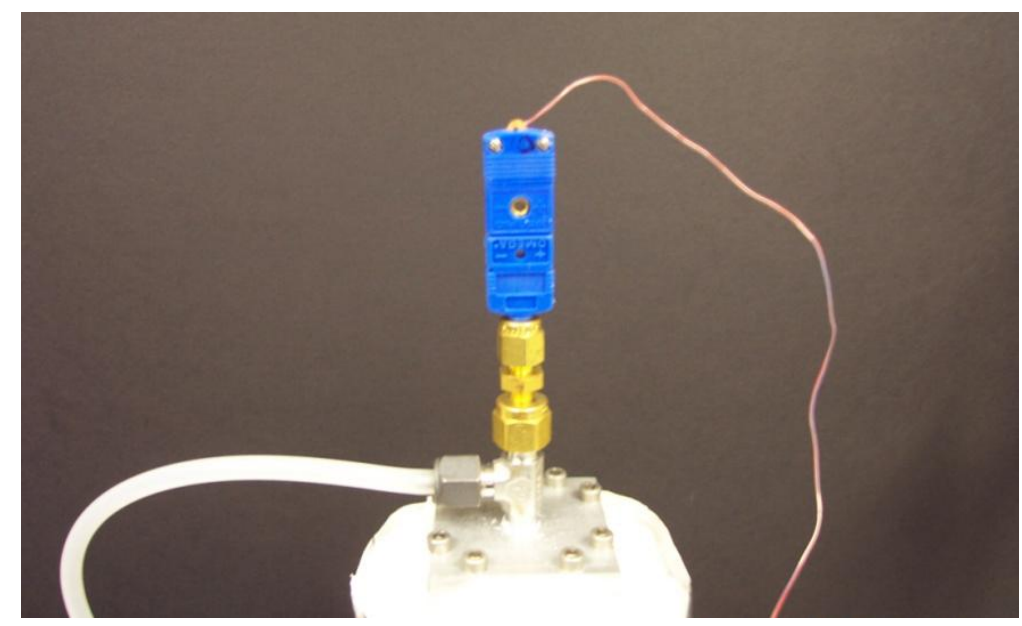

Figure A.3: Replacement Plate for Infuser Head; Thermocouple Contacts the Sample

\section{A.2 Modification 2: HTC Porosity Checks}

The infuser modification provided the opportunity to check the porosity of HTC using the method described by the manufacturer. To do this, additional fittings were installed (Figure A.4). The thermocouple in the replacement plate was removed and a $3 \mathrm{~mm}$ tube was fed into the chamber; the other end of the tube was connected to a valve, the inlet of which was immersed in a $10 \mathrm{ml}$ graduated cylinder filled with distilled water. A drip cube was placed into a $10 \mathrm{ml}$ beaker, which was set into the infuser chamber. The $3 \mathrm{~mm}$ tube was directed onto the drip cube and the chamber sealed. The port on the replacement plate was connected through a selector valve to a vacuum pump and a regulated high-pressure air source.

To infiltrate the drip cube, a vacuum was drawn in the chamber and held for two hours. The water inlet valve was opened, and water was drawn into the chamber by the vacuum; the amount of water injected was determined by the amount left in the graduated cylinder. Approximately $7 \mathrm{ml}$ water was injected per infiltration. Once the water had been injected, the vacuum was released and the chamber was slowly pressurized to $90 \mathrm{psi}$; this pressure was held for four hours. The drip cube was then removed and weighed. It was found that drip cubes infiltrated using this method could be easily handled; the water did not leak from the foam. 


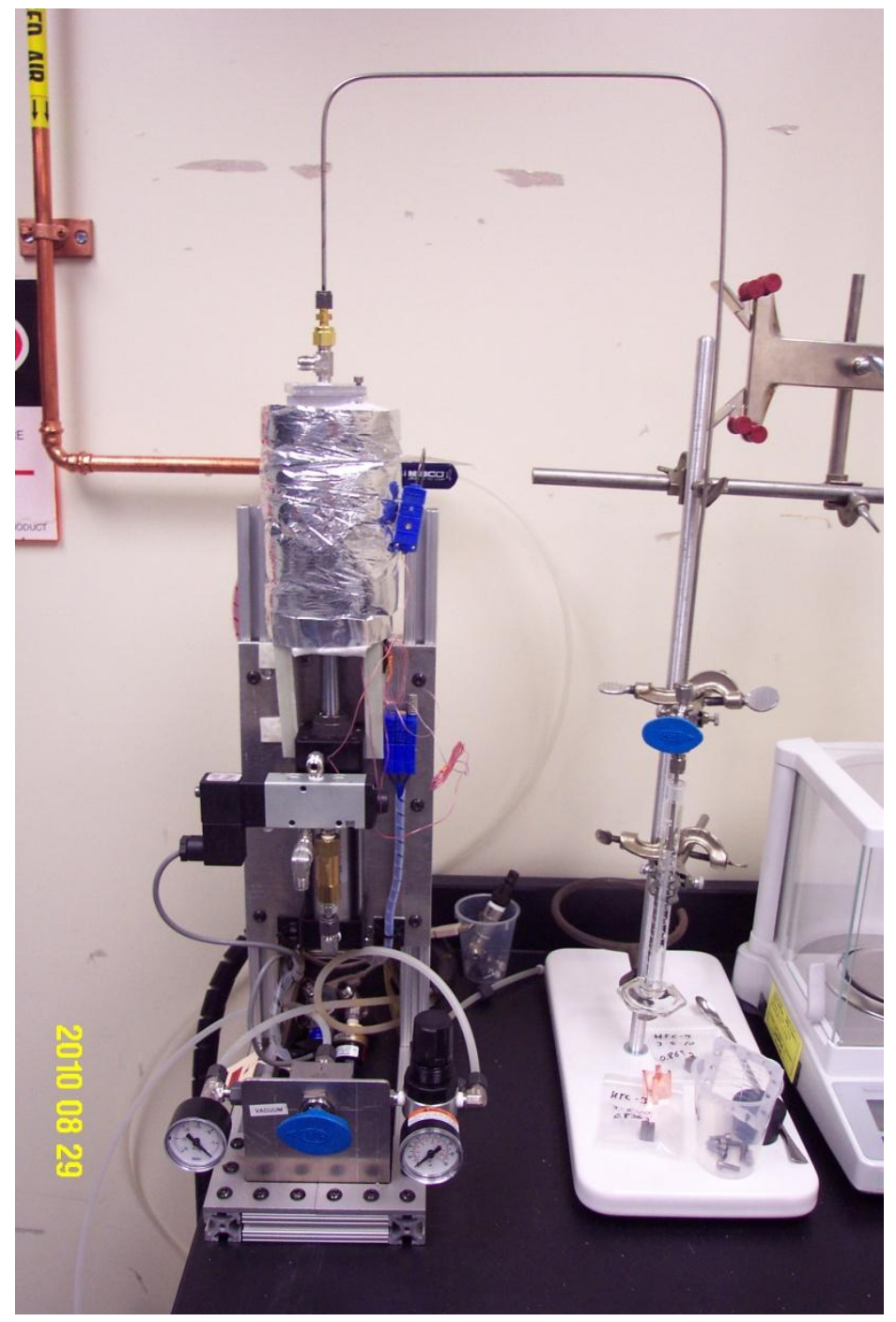

Figure A.4: Second Infuser Modification, for Infiltrating HTC Drip Cubes; Graduated Cylinder and Water Inlet Valve Are on the Equipment Stand 


\section{Appendix B: Focus Block Thermal Conductivity}

During early attempts to calculate an energy balance for the system (Sample G1002-H, constant power steady-state characterization runs) it appeared that the amount of power from the focus block was twice as high as the amount of power being put into the heater by the power supply. Power through the focus block was calculated using Equation 8, with a copper thermal conductivity value of $401 \mathrm{~W} / \mathrm{m}^{\circ} \mathrm{C}$ (Incropera and DeWitt (1985)). After calculating the implausible power value it was assumed that the focus block material was not high-purity copper, so that the conductivity was not $401 \mathrm{~W} / \mathrm{m}{ }^{\circ} \mathrm{C}$; the focus block was replaced with one that was believed to be of high purity. Additionally, the new focus block had five thermocouples attached: the front and back faces of the block had $0.5 \mathrm{~mm}$ type $\mathrm{T}$ thermocouple probes embedded in 0.5 $\mathrm{mm}$ deep grooves; a third type $\mathrm{T}$ probe was installed at the focus block center plane, $7.6 \mathrm{~mm}$ deep; and two $0.25 \mathrm{~mm}$ type $\mathrm{T}$ beads were mounted on one side, one near the front edge and the other near the back edge. Another type $\mathrm{T}$ bead was installed on the outside edge of the focus block insulator. To validate the performance of the new focus block, the sample was removed from the experimental rig and the focus block was placed in direct contact with the cold plate stem, and the active elements of the rig were insulated with a $25 \mathrm{~mm}$ thick ceramic wool blanket.

Further tests indicated that the situation had not improved with the new focus block. During a focus block validation run, the heat through the focus block was calculated to be 79.4 $\mathrm{W}$, while the input power was $30 \mathrm{~W}$. The focus block temperature drop was measured at $2^{\circ} \mathrm{C}$, and a conductivity value of $400 \mathrm{~W} / \mathrm{m}{ }^{\circ} \mathrm{C}$ was used. Based on the actual input power, the calculated 
thermal conductivity of the focus block was $151 \mathrm{~W} / \mathrm{m}{ }^{\circ} \mathrm{C}$. A cross-check using the cold plate stem indicated the heat power flowing through the sample was $28 \mathrm{~W}$; this was within $7 \%$ of the input power, and assumed to be accurate.

A numerical solver using the Euler method was written in Excel (Figure B.1), with the physical properties of the heater assembly materials as input parameters (columns F and G). It was assumed at this point that the discrepancy was being caused by large lateral losses not being considered; the actual geometry of the focus block insulator was not included in the earlier power calculations. Using the solver, the properties were varied parametrically to reproduce a focus block temperature difference that matched the measured values. It was found that setting the focus block insulation thermal conductivity to $1000 \mathrm{~W} / \mathrm{m}{ }^{\circ} \mathrm{C}$ would not reproduce the measured values; lateral losses due to insulation material or geometry were not causing the discrepancy. The solver would reproduce the measured values only if the focus block thermal conductivity were set to about $150 \mathrm{~W} / \mathrm{m}{ }^{\circ} \mathrm{C}$. At this point it was still believed that the focus block had been made of a high-purity, high conductivity copper, and that the problem was elsewhere.

\begin{tabular}{|c|c|c|c|c|c|c|c|c|c|c|c|c|c|c|}
\hline 4 & A & B & C & D & $E$ & $\mathrm{~F}$ & G & $\mathrm{H}$ & 1 & J & $\mathrm{K}$ & L & $M$ & $\mathrm{~N}=$ \\
\hline 1 & $z$ position & $T(0)$ & $P(0)$ guess & E increment & & values: & & & 0 & 0.03556 & & & & \\
\hline 2 & 0 & 362.15 & -77.77 & 704.918617 & & $\mathrm{kx}$ & 0.04 & & 0.003302 & 0.042164 & & & & \\
\hline 3 & 0.000508 & 362.110493 & -77.411901 & 704.078516 & & $h(A(x))$ & 0.0001374 & & 0.006604 & 0.048768 & & & & \\
\hline 4 & 0.001016 & 362.071168 & -77.054229 & 703.242284 & & $\mathrm{kz}$ & 385 & & 0.009906 & 0.055372 & & & & \\
\hline 5 & 0.001524 & 362.032024 & -76.696982 & 2702.409916 & & $A z$ & 0.0012645 & & 0.013208 & 0.061976 & & & & \\
\hline 6 & 0.002032 & 361.993062 & -76.340158 & 301.581406 & & TO & 362.15 & & 0.01651 & 0.06858 & & & & \\
\hline 7 & 0.00254 & 361.954281 & -75.983755 & 700.756751 & & Tn & 329 & & 0.019812 & 0.075184 & & & & \\
\hline 8 & 0.003048 & 361.915681 & -75.62777 & 7699.935947 & & $\mathrm{ds} / \mathrm{dz}$ & 0.03556 & & 0.023114 & 0.081788 & & & & \\
\hline 9 & 0.003556 & 361.877263 & -75.272203 & 699.118987 & & $\mathrm{dz}$ & 0.000508 & & 0.026416 & 0.088392 & & & & \\
\hline 10 & 0.004064 & 361.839024 & -74.91705 & 5698.305869 & & & & & 0.029718 & 0.094996 & & & & \\
\hline 11 & 0.004572 & 361.800966 & -74.562311 & 697.496587 & & $E(0)$ & 704.918617 & & 0.03302 & 0.1016 & & & & \\
\hline 12 & 0.00508 & 361.763089 & -74.207983 & 696.691137 & & & & & & & & & & \\
\hline 13 & 0.005588 & 361.725391 & -73.854064 & 695.889515 & & $q(0)$ result & 37.8609635 & & \multicolumn{2}{|l|}{$y=2 x^{*} 0.03556$} & & & & \\
\hline 14 & 0.006096 & 361.687873 & -73.500552 & 2695.091716 & & $q(L)$ result & 33.5627555 & & & & & & & \\
\hline 15 & 0.006604 & 361.650535 & -73.147445 & 694.297735 & & delta $T$ & 0.93359084 & & & & & & & \\
\hline 16 & 0.007112 & 361.613376 & -72.794742 & 2693.507569 & & & & & & & & & & \\
\hline 17 & 0.00762 & 361.576396 & $\begin{array}{ll}5 & -72.44244 \\
\end{array}$ & 692.721213 & & & & & & & & & & \\
\hline 18 & 0.008128 & 361.539596 & -72.090538 & 691.938663 & & & & & & & & & & \\
\hline 19 & 0.008636 & 361.502974 & -71.739033 & 691.159914 & & & & & & & & & & \\
\hline 20 & 0.009144 & 361.46653 & -71.387924 & 690.384962 & & & & & & & & & & \\
\hline 21 & 0.009652 & 361.430265 & -71.037208 & 689.613804 & & & & & & & & & & \\
\hline 22 & 0.01016 & 361.394178 & -70.686884 & 688.846433 & & & & & & & & & & \\
\hline 23 & 0.010668 & 361.358269 & -70.33695 & 5688.082847 & & & & & & & & & & \\
\hline 24 & 0.011176 & 361.322538 & -69.987404 & 687.323041 & & & & & & & & & & \\
\hline 25 & 0.011684 & 361.286984 & -69.638244 & 686.567011 & & & & & & & & & & \\
\hline 26 & 0.012192 & 361.251608 & -69.289468 & 685.814753 & & & & & & & & & & \\
\hline & $\rightarrow M[$ euler & focus eul & ler test $\quad \cdots$ She & eet3 3 & & & & & & & & & & 1 1 \\
\hline $\operatorname{Rea}$ & & & & & & & & & & & & & & \\
\hline
\end{tabular}

Figure B.1: Excel Euler Parametric Solver Used to Model the Heater Block Performance 
A thermal analysis was then performed using Dassault Solidworks. One fourth of the complete heater block assembly was modeled (Figure B.2), including thermal resistances between elements and material properties. Boundary conditions were primarily convective losses or symmetry planes; the focus block face was given a constant flux output. The goal of the analysis was to reproduce the temperature distributions recorded during two constant-power focus block validation runs of 3.0 and $9.0 \mathrm{~W} / \mathrm{cm}^{2}$ input.

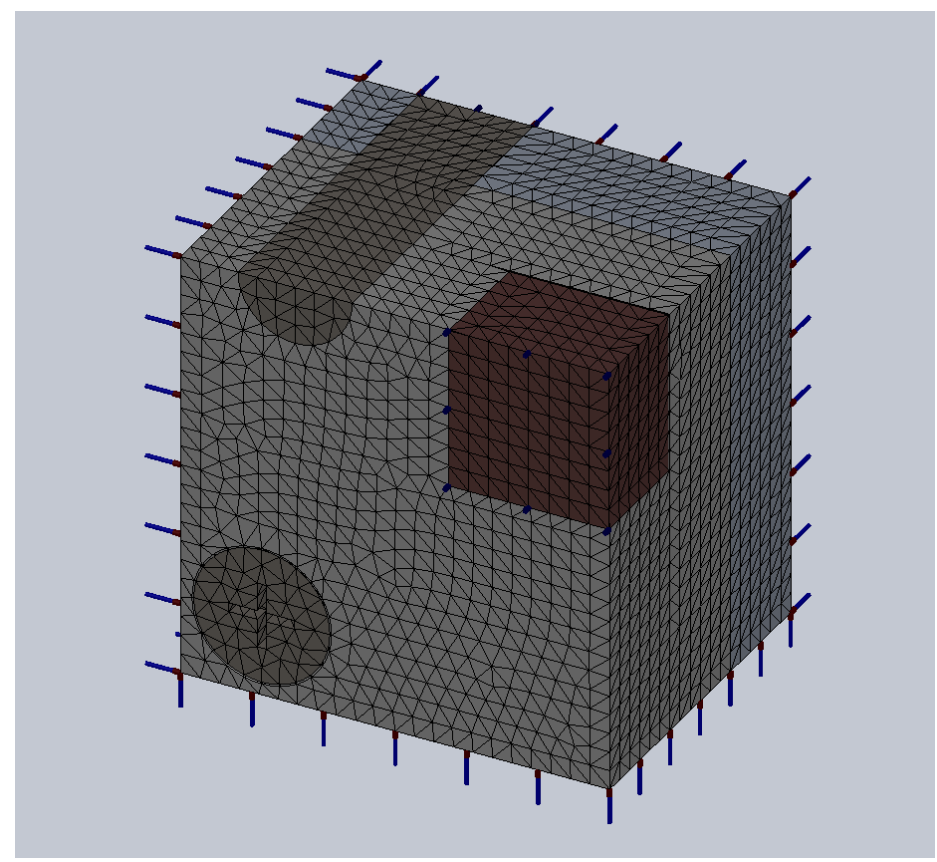

Figure B.2: Solidworks Mesh of Heater Block Assembly; One-Fourth of the Assembly was Modeled

The material properties and boundary conditions were initially modified using the 3.0 $\mathrm{W} / \mathrm{cm}^{2}$ data to achieve agreement between the model and the experimental results; no significant deviations from actual values were required. These established property values and boundary conditions were then used to model the $9.0 \mathrm{~W} / \mathrm{cm}^{2}$ data; the only significant modification from the $3.0 \mathrm{~W} / \mathrm{cm}^{2}$ study was to increase the thermal resistance between the heater element and the focus block from 0.000005 to $0.05^{\circ} \mathrm{C} / \mathrm{W}$. With this change, the model fit the $9.0 \mathrm{~W} / \mathrm{cm}^{2}$ data with 98\% accuracy. This suggested that the model accurately portrayed the thermal response of the heater block assembly. 
In order to get the model's temperature difference across the focus block to agree with experimental results, the thermal conductivity value of copper had to be reduced to about $1 / 3$ the published value, to $133 \mathrm{~W} / \mathrm{m}{ }^{\circ} \mathrm{C}$. Figure B.3 shows the temperature gradient across the focus block and the high temperature in the heater element, caused by the resistances between the heater element and the focus block and of the focus block itself. Adjusting the focus block's lateral loss by increasing the conductivity of the forward insulator did not result in the desired difference, nor did high power output or large thermal resistance between the element and the focus block. Based on these results, it became obvious that the focus block material was not high thermal conductivity copper.

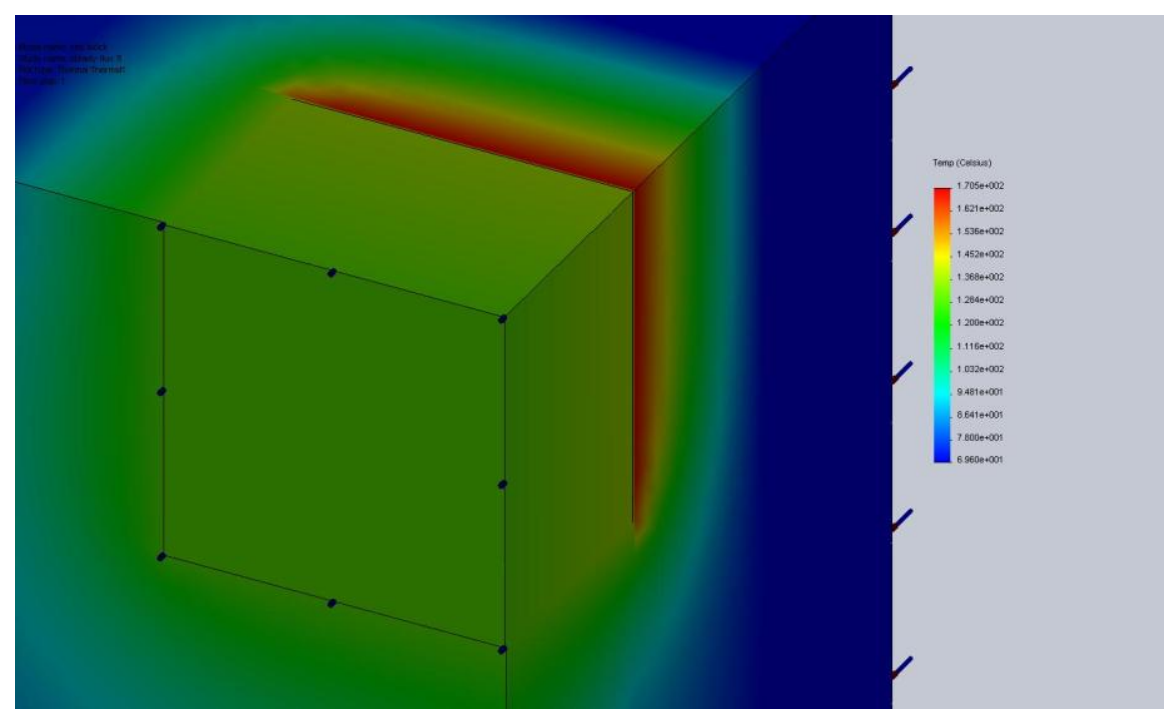

\section{Figure B.3: Solidworks Analysis Results of the 9.0 W/cm² Run; Focus Block Conductivity Was Set to $133 \mathrm{~W} / \mathrm{m}^{\circ} \mathrm{C}$ to Reproduce Experimental Measurements}

A piece of copper with known properties (Alloy 101; oxygen-free, 99.1\% pure) was obtained and a new focus block was fabricated. The center plane thermocouple was eliminated; the face thermocouples were embedded in the block, $0.25 \mathrm{~mm}$ from the faces and $7.6 \mathrm{~mm}$ deep, rather than in grooves on the surface. The system was set to run a typical chilled run (circulation bath $65^{\circ}$, input power $78 \mathrm{~W}$ ) and allowed to reach steady state; the trip set point was set to $110^{\circ} \mathrm{C}$. After seven hours, the maximum temperature reached was $100^{\circ}$; the temperature difference across the focus block was $1.8^{\circ} \mathrm{C}$. With a measured input power of $79.4 \mathrm{~W}$, the calculated focus block 
output power was $78.7 \mathrm{~W}$ using a thermal conductivity of $390 \mathrm{~W} / \mathrm{m}{ }^{\circ} \mathrm{C}$, and the cold plate stem power was calculated to be $78.2 \mathrm{~W}$ using a thermal conductivity of $355 \mathrm{~W} / \mathrm{m}{ }^{\circ} \mathrm{C}$. These values were then used to perform all analyses for the remainder of this project. 


\section{Appendix C: Uncertainty Analyses}

\section{C.1 Thermocouple Calibration and Temperature Measurement Uncertainties}

There are four sources of uncertainty in temperature measurements taken with a calibrated thermocouple: the uncertainty of the standard temperature measurement to which the thermocouple was calibrated, the temperature range in which the reading was considered steady, the uncertainty of the calibration fit, and the uncertainty due to the randomness of thermocouple reading itself, which will be referred to here as "drift."

For the thermocouple calibrations, a Hart Scientific 5628 RTD and a 1502A RTD reader were used to define the standard temperature, which had a combined listed uncertainty of $\pm 0.033^{\circ} \mathrm{C}$. The method used to fix a temperature set point was to have LabVIEW command the calibration bath (Hart Scientific 7320) to some set point temperature. When the RTD reading had stabilized to within $\pm 0.02^{\circ} \mathrm{C}$, which typically took ten minutes, LabVIEW began recording the thermocouple temperatures. Four groups of type T thermocouples (A through D) were calibrated; the typical number in each group was 20 thermocouples. Each thermocouple group was calibrated from 10 to $100^{\circ} \mathrm{C}$ in $5^{\circ} \mathrm{C}$ increments, with between 100 and 300 data points recorded for each thermocouple at each temperature. The data files were reduced by taking the averages and standard deviations of 100 points from each thermocouple. The averages were used to calculate the calibration fit coefficients using a least-squares method in MATLAB (polyfit) and Excel 
(LINEST), and the standard deviations of the means were used to find the thermocouple drift uncertainties.

The calibration fits almost universally turned out to be fourth order, based on the minimum sum-of-squared-residuals (SSR). The ones that weren't fourth order were second order, with a calculated SSR difference between second and fourth orders less than 0.001. All thermocouples were calibrated using fourth order fits. The group A thermocouples were calibrated twice; the largest difference between any two fits in the first and second group A runs was found to be within $0.1 \%$; it was decided that multiple runs on subsequent thermocouple groups would be unnecessary. The calibration coefficients gave residuals within $0.04^{\circ}$ for all thermocouples. Figure C.1 shows the residual plot for the five group B thermocouples that were embedded within sample G1002-H. The apparent randomness and the small range (-0.033 to $0.026^{\circ} \mathrm{C}$ ) of the residuals indicates that the fourth-order fit accurately recreates the standard temperature (RTD) distribution.

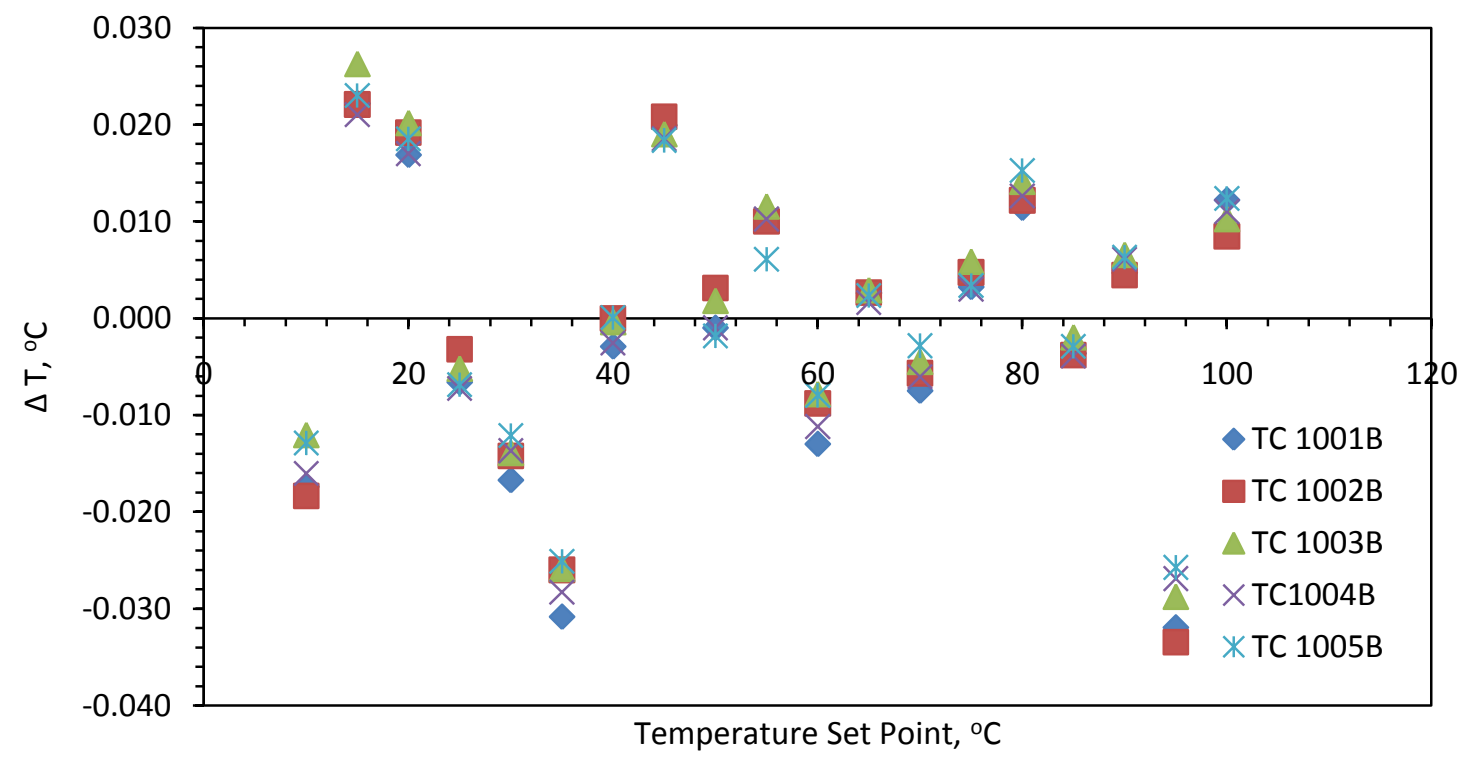

\section{Figure C.1: Residual Plot of the Fourth Order Fits for Thermocouples 1001B-1005B, Used in Sample G1002-H (HTC/PG)}


The drift uncertainty was calculated by taking the standard deviation of the mean value calculated from the first 100 data points taken for each thermocouple at each temperature set point; two standard deviations $(2 \sigma)$ gives a $95 \%$ probability that the measured value is within the confidence interval $(\mathrm{CI})$ of the actual value. The $2 \sigma$ values were calculated for all the TCs at all the temperatures. In the temperature range of interest $\left(50\right.$ to $\left.100^{\circ} \mathrm{C}\right)$ every single one (except for obviously defective thermocouples) was below 0.08 , which is much greater than $95 \%$, but the 95\% value was used. Based on the two calculated uncertainties and the standard uncertainty, using the equation

$$
\delta_{\text {tot }}=\sqrt{\delta_{\text {fit }}^{2}+\delta_{\text {drift }}^{2}+\delta_{\text {std }}^{2}+\delta_{\text {steady }}^{2}}
$$

The temperature measurement uncertainty range was found to be

$$
\delta_{\text {tot }}=\sqrt{0.04^{2}+0.08^{2}+0.033^{2}+0.02^{2}}= \pm 0.0974^{\circ} \mathrm{C}
$$

It was decided to use the uncertainty range $\pm 0.1^{\circ} \mathrm{C}$, which included all observed deviations.

\section{C.2 Temperature Difference Uncertainties}

Many of the calculations performed for this project required finding the temperature difference between two thermocouples. The uncertainty of those calculations is found by

$$
\delta_{\Delta T}=\sqrt{\delta_{T_{1}}^{2}+\delta_{T_{2}}^{2}}
$$

Having determined that the uncertainty of each temperature measurement was $\pm 0.1^{\circ} \mathrm{C}$, the uncertainty for measured temperature differences was found to be $\pm 0.141^{\circ} \mathrm{C}$.

\section{C.3 Time Step Uncertainty}

The uncertainty of the LabVIEW scan time step was required to determine of the accuracy of the calculated amount of energy stored sensibly in each sample component, and the 
heating and cooling rates of the samples. The time steps are a consequence of the way LabVIEW was set up; because of the number of measurements taken and components controlled (primarily the power supply and the circulation bath), the scan rate was given a two-second delay to complete all operations. The measurements and operations themselves also added a small finite time to each scan.

During the heating rate analyses, the time steps of each cycle through heating transition were averaged. The average time step was then used to calculate the temperature change rate (Equation 6, Chapter 2):

$$
\frac{d T_{j}}{d t}=\frac{T_{j, t+1}-T_{j, t}}{\overline{\Delta t}}
$$

Because the period over which the time steps were averaged was fairly short-heating transition times were on the order of 260 seconds for the $2.3 \mathrm{~W} / \mathrm{cm}^{2}$ runs and $90 \mathrm{~s}$ for the $6.0 \mathrm{~W} / \mathrm{cm}^{2}$ runsthe Student's t-distribution method of determining the uncertainty range was used. The typical time step value was 2.4 seconds, giving time step sample populations ( $n$ ) of 65 and 23 for the 2.3 $\mathrm{W} / \mathrm{cm}^{2}$ and $6.0 \mathrm{~W} / \mathrm{cm}^{2}$ runs, respectively; the standard deviation for the time steps in the heating transition periods was typically 0.5 second. The t-distribution uncertainty range is found using Equation C4 (Moore and McCabe (2006)):

$$
t^{*}\left(\frac{\sigma}{\sqrt{n}}\right)=\text { margin of error }(C I)
$$

where $t^{*}$ is defined by Moore and McCabe as the "value for the $t(n-1)$ density curve between $-t^{*}$ and $t^{*}, " \sigma$ is the standard deviation of the mean, and $n$ is the sample population size. For a $95 \%$ CI, $t^{*}$ for $n=65$ is 2.00 , and for $n=23, t^{*}=2.07$. The CI for the $2.3 \mathrm{~W} / \mathrm{cm}^{2}$ runs is on the order of $0.12 \mathrm{~s}$; for the $6.0 \mathrm{~W} / \mathrm{cm}^{2}$ runs, the CI is on the order of 0.21 seconds. Because most of the experiments of interest operated at the $6.0 \mathrm{~W} / \mathrm{cm}^{2}$ flux, the time steps were considered to be $2.40 \pm 0.21$ seconds, meaning the error was $8.8 \%$ of the average time step. 


\section{C.4 Temperature Change Rate Uncertainties}

The temperature change rate uncertainties were evaluated using error propagation methods ; the uncertainties produced were on the order of $34 \%$ of typical readings due to relatively large uncertainties in temperature differences and time steps. However, because each sample was subjected to numerous cycles during the experiments, it was clear that the heating and cooling rates did not vary by such a large amount. It was decided to use the measured values to calculate the temperature change rate uncertainties.

The student's t-distribution method described in Appendix C.3 was applied. Typical experimental runs were cycled four times, and it was known that the heating rates of the first cycles were artificially long so each experiment had two degrees of freedom $(n-1)$ for these evaluations. For a 95\% CI with 2 degrees of freedom, the $t^{*}$ value is 4.303 ; the mean standard deviation for the $\mathrm{G} 1002-\mathrm{H} 6.0 \mathrm{~W} / \mathrm{cm}^{2}$ chilled run heating rates was 0.0017 , with a mean of $0.086^{\circ} \mathrm{C} / \mathrm{s}$. Using Equation C4, the calculated $95 \% \mathrm{CI}$ was 0.004 . For the G1001-H $6.0 \mathrm{~W} / \mathrm{cm}^{2}$ insulated run, a total of seven cycles were performed, giving six degrees of freedom, a mean heating rate of $0.177^{\circ} \mathrm{C} / \mathrm{s}$, and a standard deviation of 0.004 ; the t value was 2.447 , which gave a $95 \% \mathrm{CI}$ of 0.004 . The $\mathrm{G} 1002-\mathrm{H} 6.0 \mathrm{~W} / \mathrm{cm}^{2}$ insulated run (mean heating rate $0.126^{\circ} \mathrm{C} / \mathrm{s}$ ) gave the same result. Based on these results, the uncertainty in temperature change rates was a maximum of $4.5 \%$ of the mean rates.

\section{C.5 Flux Meter Heat Transfer Uncertainties}

The heat transfer across the flux meters were calculated using Equation 8, repeated here:

$$
q=\frac{k A}{L}\left(T_{1}-T_{2}\right)
$$

To calculate the heat transfer uncertainties, the error propagation formula for multiplied values was used: 


$$
\delta Z(x, y)=Z(x, y) \sqrt{\left(\frac{\delta x}{x}\right)^{2}+\left(\frac{\delta y}{y}\right)^{2}}
$$

The temperature difference uncertainty was considered in Appendix C.2, and was found to be $\pm 0.141^{\circ} \mathrm{C}$. Length measurements had uncertainties of $\pm 1.27 * 10^{-5} \mathrm{~m}$. For the G1002-H 9.0 $\mathrm{W} / \mathrm{cm}^{2}$ chilled focus block validation experiment discussed in Appendix B, the average focus block heat transfer was $78.70 \mathrm{~W}$. The resulting uncertainty was

$$
\delta q=78.70 \sqrt{\left(\frac{0.141}{1.81}\right)^{2}+\left(\frac{1.27 * 10^{-5}}{0.03580}\right)^{2}+\left(\frac{1.27 * 10^{-5}}{0.03582}\right)^{2}+\left(\frac{1.27 * 10^{-5}}{0.01146}\right)^{2}}
$$

giving $\pm 6.14 \mathrm{~W}$, or $7.8 \%$ of the reading.

By the same method, the cold plate stem heat transfer uncertainty for the same experiment was

$$
\delta q=78.20 \sqrt{\left(\frac{0.141}{1.72}\right)^{2}+\left(\frac{1.27 * 10^{-5}}{0.03587}\right)^{2}+\left(\frac{1.27 * 10^{-5}}{0.03587}\right)^{2}+\left(\frac{1.27 * 10^{-5}}{0.01}\right)^{2}}
$$

giving $\pm 6.41 \mathrm{~W}$, or $8.2 \%$ of the reading.

\section{C.6 Sensible Energy Storage Rate Uncertainties}

The heat stored sensibly in the sample components were calculated using Equation C7:

$$
\dot{E}_{\text {st,sens }}=\sum_{i} m_{i} c_{i} \frac{\partial T_{i}}{\partial t}
$$

The mass of each component was determined using the Mettler-Toledo PB403-S/FACT precision balance. The measurement uncertainty is listed as $+1 /-3 \mathrm{mg}$ on the calibration certificate; component masses were recorded to $\pm 5 \mathrm{mg}$. Masses and specific heat capacities (considered absolute) for the sample components are given in Table 2.3. The temperature change rate uncertainty was discussed in Appendix C.4, and was found to be $4.5 \%$ of the reading.

Using the calculated energy storage rate of the holder shell of sample G1002-H during the fourth cycle of the $6.0 \mathrm{~W} / \mathrm{cm}^{2}$ chilled run as an example, the average storage rate was $2.06 \mathrm{~W}$; 
the average temperature rise rate was $0.03^{\circ} \mathrm{C} / \mathrm{s}$. Applying Equation E6 to the storage rate of each of the materials in the sample, the storage rate uncertainties of each component are:

$$
\begin{gathered}
\delta \dot{E}_{\text {shell }}=\dot{E}_{\text {shell }} \sqrt{\left(\frac{\delta m_{\text {shell }}}{m_{\text {shell }}}\right)^{2}+\left(\frac{\delta \dot{T}_{\text {shell }}}{\dot{T}_{\text {shell }}}\right)^{2}} \\
\delta \dot{E}_{f}=\dot{E}_{f} \sqrt{\left(\frac{\delta m_{f}}{m_{f}}\right)^{2}+\left(\frac{\delta \dot{T}_{f}}{\dot{T}_{f}}\right)^{2}} \\
\delta \dot{E}_{\text {cap }}=\dot{E}_{\text {cap }} \sqrt{\left(\frac{\delta m_{\text {cap }}}{m_{\text {cap }}}\right)^{2}+\left(\frac{\delta \dot{T}_{\text {cap }}}{\dot{T}_{\text {cap }}}\right)^{2}} \\
\delta \dot{E}_{\text {plate }}=\dot{E}_{\text {plate }} \sqrt{\left(\frac{\delta m_{\text {plate }}}{m_{\text {plate }}}\right)^{2}+\left(\frac{\delta \dot{T}_{\text {plate }}}{\dot{T}_{\text {plate }}}\right)^{2}}
\end{gathered}
$$

where $\dot{T}$ is the temperature change rate; the subscripts shell, $f$, cap and plate refer to the sample holder shell, sample foam, holder end caps and end plates, respectively. Table C.1 shows the average calculated energy storage rate and temperature change rate for each component.

\section{Table C.1 Sample G1002-H Component Storage and Heating Rates}

\begin{tabular}{ccc}
\hline Component & $\begin{array}{c}\text { Storage Rate } \\
(\mathbf{W})\end{array}$ & $\begin{array}{c}\text { Temperature Rise Rate } \\
\left({ }^{\mathbf{0}} \mathbf{C} / \mathbf{s}\right)\end{array}$ \\
\hline Shell & 2.06 & 0.03 \\
Foam & 4.22 & 0.06 \\
End Cap & 3.05 & 0.06 \\
End Plate & 0.36 & 0.06 \\
\hline
\end{tabular}

From the values in Table C.1, the component storage rate uncertainties are $\pm 0.09 \mathrm{~W}$ for the shell, $\pm 0.19 \mathrm{~W}$ for the foam, $\pm 0.02 \mathrm{~W}$ for the end plates, and $\pm 0.14 \mathrm{~W}$ for the end caps. These values are about $4.5 \%$ of the reading for each component, which is expected considering the magnitude of the temperature change uncertainties compared to the mass uncertainties. 
The uncertainty for added or subtracted values $Z=x+y$ is calculated using Equation C12:

$$
\delta Z(x, y)=\sqrt{(\delta x)^{2}+(\delta y)^{2}}
$$

The individual uncertainties are combined to give the overall uncertainty of the energy storage rate for the sample,

$$
\delta \dot{E}_{\text {st }, \text { sens }}=\sqrt{\left(\delta \dot{E}_{\text {shell }}\right)^{2}+\left(\delta \dot{E}_{f}\right)^{2}+\left(\delta \dot{E}_{\text {cap }}\right)^{2}+\left(\delta \dot{E}_{\text {plate }}\right)^{2}}
$$

giving $\pm 0.25 \mathrm{~W}$, or $2.6 \%$ of the calculated total sensible energy stored in the sample components through heating transition $(9.694 \mathrm{~W})$. 


\section{Appendix D: Experiment SOP}

This Appendix lists the steps that the experimenter is to follow to generate data for an

experimental run. These steps are not sample-specific, in that the steps apply to every experimental run regardless of the sample or the boundary conditions specified. The SOP was posted next to the experiment for easy operator access.

(Assumes a previously prepared sample installed in the insulated holder)

1. Turn off air supply using needle valve (black knob on air valve)

2. Cycle air cylinder to relieve excess air pressure

3. Remove insulation blanket

4. Open needle valve 1 turn; retract air cylinder; close needle valve

5. Remove the four $1 / 2$ " tie rod nuts from the right end (top) of apparatus

6. Disconnect focus block TCs $(1019,1020)$

7. Slide air cylinder/heater assembly out (remove from tie rods and guide pins), set aside

a. Ensure TC, power leads do not snag

8. Align sample insulation guide holes with the guide pins and slide the sample onto the pins

9. Connect the sample thermocouples to the correct channels

10. Install and connect the backside insulator (if required) 
11. Reinstall the air cylinder/heater assembly (slide back onto guide pins, tie rods) until it is seated against the locknuts

12. Reinstall the $1 / 2$ " nuts onto the tie rods (finger tight is good enough)

13. Reconnect focus block TCs

14. Open air supply needle valve

15. Apply system contact load: stroke air cylinder out

16. Replace blanket

17. Generate the configuration file for LabVIEW
a. TCs: channel, label
b. TC fit curve data
c. Other channels (DC power, load cell, flow meter)

18. Turn on the equipment
a. Agilent 34980A DAQ
b. HP 6030A power supply
c. Neslab RTE 17 chill bath:

i. check that "Computer" indicator is lit
d. computer
e. Start LabVIEW
f. Open file "TES with PID 5.3.vi"

19. Start the LabVIEW VI "TES with PID 5.3"
a. Click the "Program Start" button
b. Select Chilled or Insulated Run
c. Enter the Chill Bath Set Point
d. Select Constant Power or Constant Temperature
i. Constant Power: enter power set point
ii. Constant Temperature: enter heater temperature 
e. Enter the Trip Point temperature

f. Enter the Recycle Point temperature

g. Select the configuration file to be used

h. Enter the name of the data file

20. When chill bath reaches set point, click "OK"

21. Set chill bath flow rate to desired level using the HX needle valve (green knob) on the apparatus; check flow rate; close the HX ball valve (blue knob) if required

22. Set contact load to the desired level using the air regulator

23. Check TC readings to ensure proper function

24. Once settings are correct and TCs are working, click "POWER ON/OFF" to start the heater power (timing depends on the experimental conditions)

25. Open HX ball valve to start coolant flow (timing depends on the experimental conditions)

26. Record data as required

a. Monitor readings for indications of problems

27. When finished with the run, click "POWER ON/OFF" to stop the heater power (if it is still on)

28. Click "STOP"

29. Wait for LabVIEW to finish closing the program:
a. Chill bath will turn off
b. LabVIEW front panel will disable
c. LabVIEW will save data to Excel file

30. Turn off equipment
a. Agilent 34980A DAQ
b. HP 6030A power supply

31. Back up data file to external hard drive

a. Data files are saved in "My Documents" 


\section{Appendix E: Sample Configuration File}

This Appendix lists a typical configuration file. The configuration files were used to configure the system for a particular sample under a particular type of boundary conditions. The file shown below configured the experiment's LabVIEW code and the data acquisition unit (DAQ) to label and scan a selected set of channels, and apply the calibration coefficients to the results before they are recorded in the Excel data file.

Each channel configuration consists of five lines. The first line is the channel name (e.g. [1001]), as assigned by the DAQ; the second line is the label assigned to the channel when it is recorded in the Excel data file; the last three lines are the calibration formula input (FormInput) and output (FormOutput) assignments, and formula (Formula) containing the coefficients used to calibrate the raw signal from the channel to the calibrated data required. Note that some of the channels do not have calibration data applied; for these channels, the raw data were recorded.

Of the 24 channels assigned, the first nine $(1001-1006,1009-1011)$ are for the sample and holder; the next two $(1013,1016)$ measure environmental temperatures; the next seven $(1019$, $1020,1022-1026)$ are located in the heater block assembly; four $(5016,5017,5019,5020)$ are mounted in the cold plate; and the last two are for the load cell (5022) and the flow meter (5025), respectively. The file listed in this Appendix configured LabVIEW and the DAQ for tests on Sample G1002-H (HTC/PG) with a constant power input boundary and an actively cooled output boundary, and was assigned the name "G1002-H chilled.ini." 
As discussed in Chapter 2, the configuration files were used to keep track of the necessary data changes for each sample. Each sample had a unique set of thermocouples embedded and sometimes tested samples were re-tested due to changes in the experimental hardware; using the configuration files made these sample changes much less confusing.

[1001]

Label="Sample 0.087"

Formula $=\mathrm{a}=0.03422058047776+1.01382804045624 * \mathrm{a} 2-0.00050864646039 * \mathrm{a} 2^{\wedge} 2+$ $0.00000603646905^{*} \mathrm{a} 2^{\wedge} 3-0.00000002459935^{*} \mathrm{a} 2^{\wedge} 4 ; "$

Formlnput=a2

FormOutput=a

[1002]

Label="Sample 0.337"

Formula $=" \mathrm{~b}=0.06119951636452+1.01203868665424 * \mathrm{~b} 2-0.00046166244637^{*} \mathrm{~b} 2^{\wedge} 2+$ $0.00000553288537 * b 2^{\wedge} 3-0.00000002264407 * b 2^{\wedge} 4 ; "$

FormInput=b2

FormOutput=b

[1003]

Label="Sample 0.586"

Formula $=" \mathrm{c}=0.05335778856778+1.01270377633514 * \mathrm{c} 2-0.00046851403610^{*} \mathrm{c} 2^{\wedge} 2+$ $0.00000551082535^{*} c 2^{\wedge} 3-0.00000002223216^{*} c 2^{\wedge} 4 ; "$

Formlnput=c2

FormOutput=c

[1004]

Label="Sample 0.840"

Formula $=" \mathrm{~d}=0.07999482696296+1.01147619819380^{*} \mathrm{~d} 2-0.00043945370021^{*} \mathrm{~d} 2^{\wedge} 2+$ $0.00000523743591 * \mathrm{~d} 2^{\wedge} 3-0.00000002132619 * \mathrm{~d} 2^{\wedge} 4 ;$ "

FormInput=d2

FormOutput $=\mathrm{d}$

[1005] 


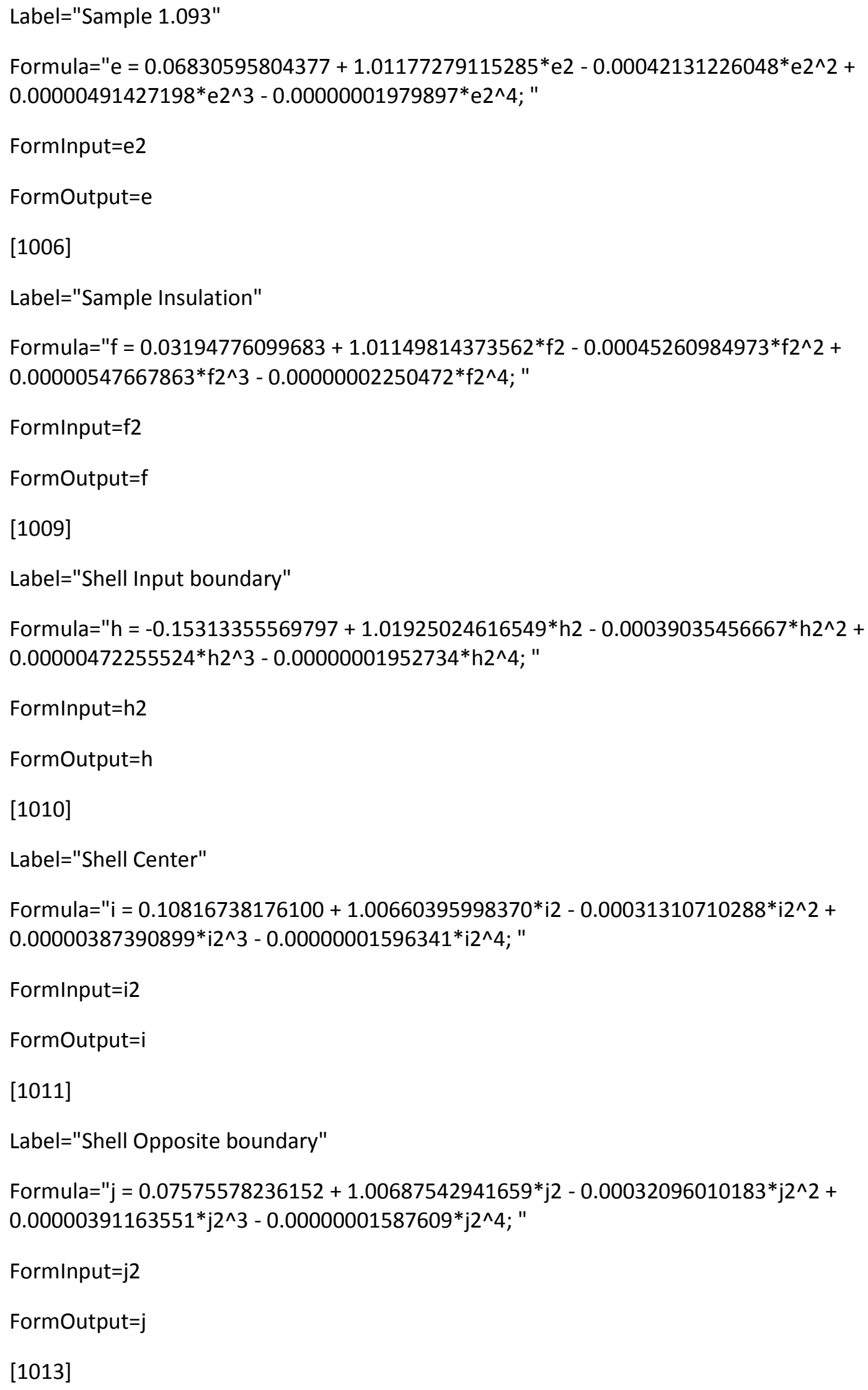




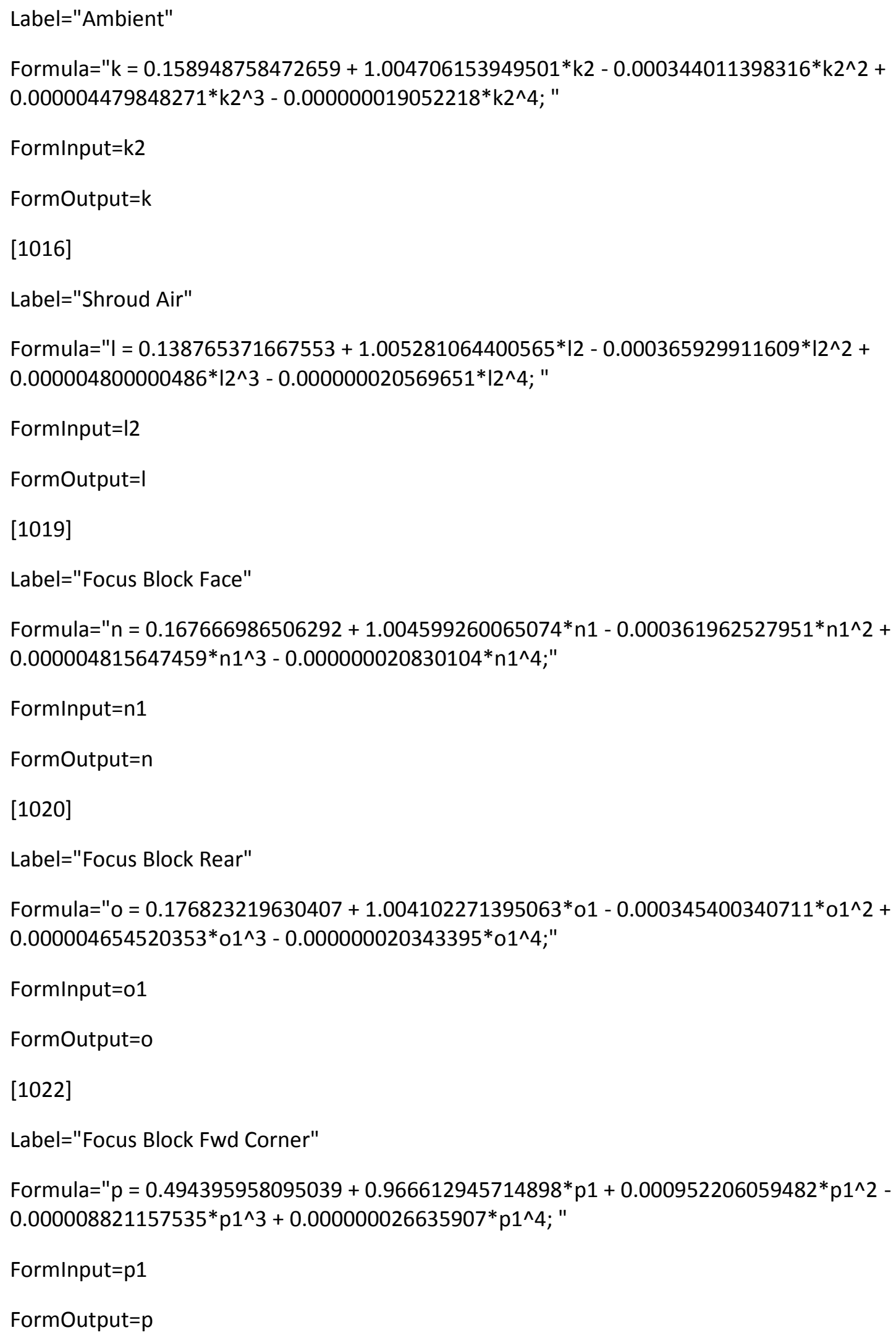




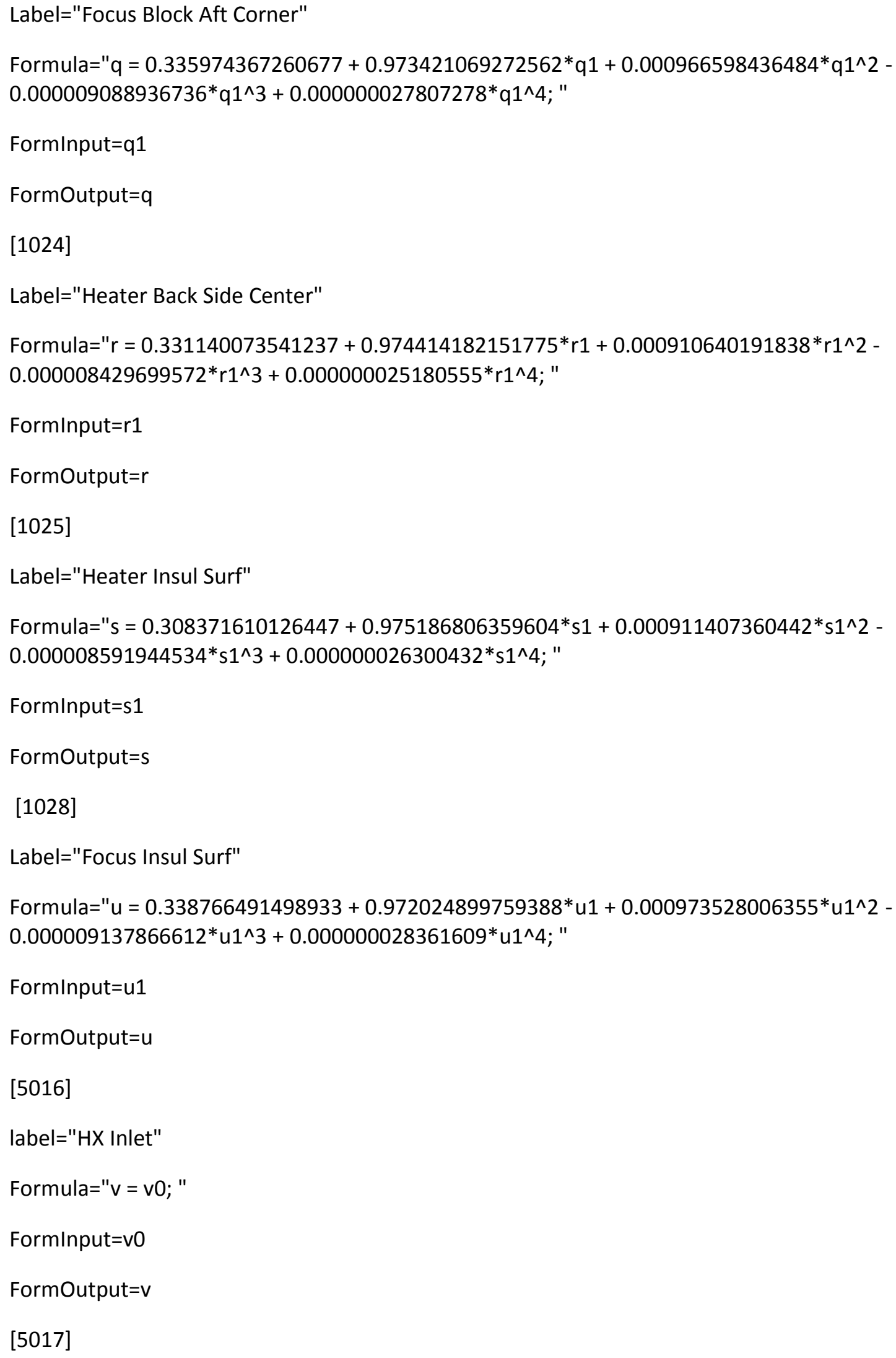




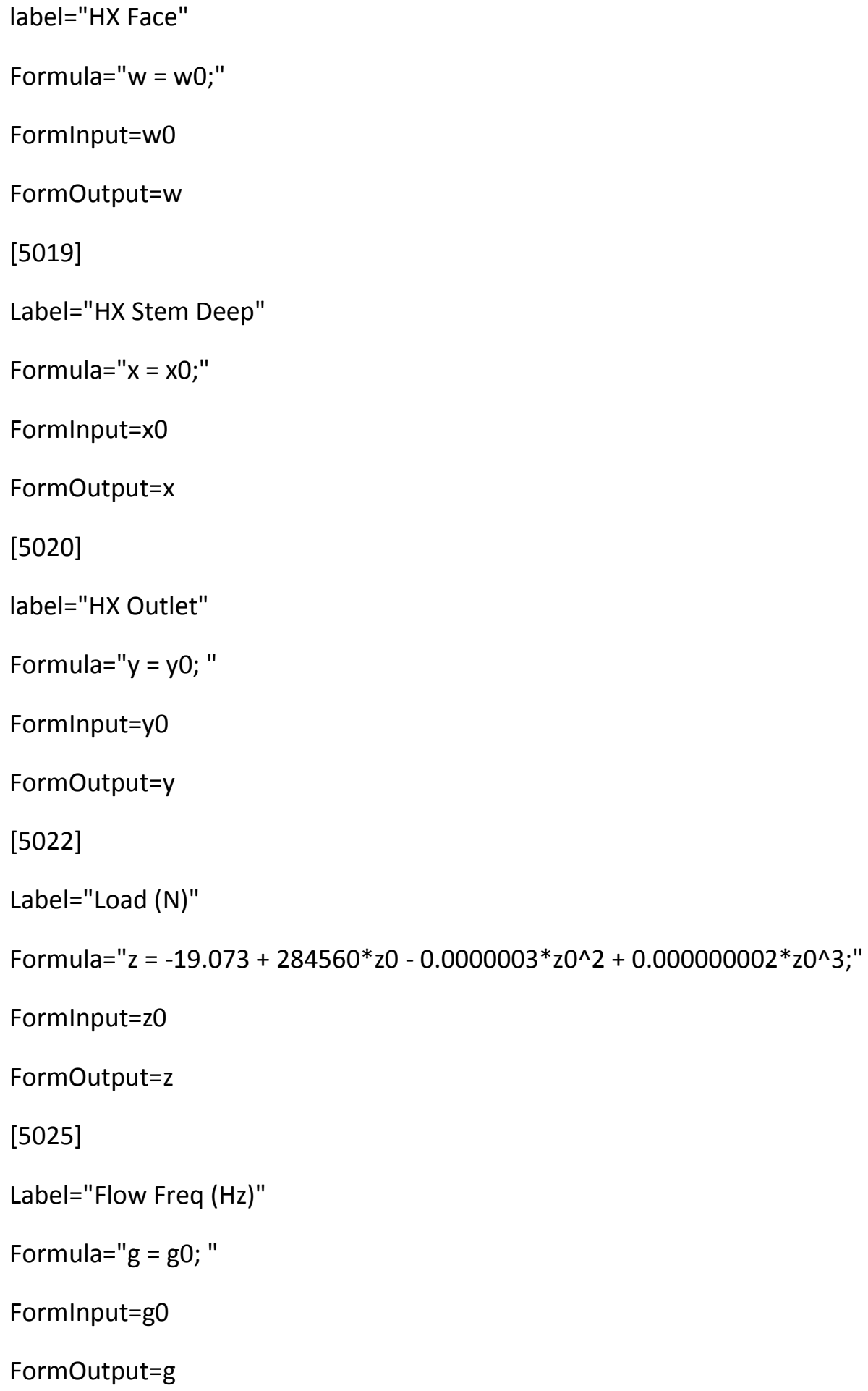

